University of Louisville

ThinkIR: The University of Louisville's Institutional Repository

Electronic Theses and Dissertations

$5-2009$

\title{
The effect of teacher leader interactions with teachers on student achievement: a predictive study.
}

Winifred Riney Cohron

University of Louisville

Follow this and additional works at: https://ir.library.louisville.edu/etd

\section{Recommended Citation}

Cohron, Winifred Riney, "The effect of teacher leader interactions with teachers on student achievement: a predictive study." (2009). Electronic Theses and Dissertations. Paper 262.

https://doi.org/10.18297/etd/262

This Doctoral Dissertation is brought to you for free and open access by ThinkIR: The University of Louisville's Institutional Repository. It has been accepted for inclusion in Electronic Theses and Dissertations by an authorized administrator of ThinkIR: The University of Louisville's Institutional Repository. This title appears here courtesy of the author, who has retained all other copyrights. For more information, please contact thinkir@louisville.edu. 


\title{
THE EFFECT OF TEACHER LEADER INTERACTIONS \\ WITH TEACHERS ON STUDENT ACHIEVEMENT:
}

\section{A PREDICTIVE STUDY}

\author{
By \\ Winifred Riney Cohron \\ B.A. Brescia College, 1974 \\ M.R.E Institute of Pastoral Studies, Loyola University, Chicago, 1983
}

\author{
A Dissertation \\ Submitted to the Faculty of the \\ Graduate School of the University of Louisville \\ and \\ Graduate Studies and Research at Western Kentucky University \\ In Partial Fulfillment of the Requirements \\ for the Degree of
}

\author{
Doctor of Philosophy \\ Department of Leadership, Foundations, and Human Resource Education \\ University of Louisville \\ and \\ College of Education and Behavioral Sciences \\ Western Kentucky University
}

May 2009 


\title{
THE EFFECT OF TEACHER LEADER INTERACTIONS \\ WITH TEACHERS ON STUDENT ACHIEVEMENT: \\ A PREDICTIVE STUDY
}

\author{
By \\ Winifred Riney Cohron \\ B.A. Brescia College, 1974 \\ M.R.E Institute of Pastoral Studies, Loyola University, Chicago, 1983
}

A Dissertation Approved on

March 30, 2009

By the following Dissertation Committee: 


\section{DEDICATION}

This dissertation is dedicated to my husband and best friend

\section{Gene Manning Cohron}

who shows me everyday what love looks like. 


\section{ACKNOWLEDGEMENTS}

I would like to express gratitude to the members of my dissertation committee for their unflagging support in this research process. To Dr. Fiene, "thank you" seems inadequate in light of your role in this endeavor over a span of several years. The cohort gatherings you hosted provided both intellectual and emotional support, as well as lots of stress-relieving laughter. You continue to be a source of inspiration for me. To Dr. Petrosko, thank you for your consistently prompt and kind responses to the myriad statistical questions sent your way. Your advice was always expressed with great care and clear logic. To Dr. Schlinker, thank you for being such a wonderful role model, mentor, and friend through these many years. To Dr. Spall, thank you for the opportunity to discover the richness that language about lived experience can bring to research. To Dr. Sullivan, thank you for bringing your skill and practice in working with teachers to this project. To Dr. Meg Sidle, thank you for guiding me through the intricacies of SPSS. To Dr. Steve Miller, thanks for getting me started. To all of you, thank you for the time and thought you gave in reading, listening, and advising. I am in your debt.

To the library staff at WKU, specifically, Selina Langford, Beth Knight, and Amy Wilk, thank you for making resource retrieval so uncomplicated. To Clarkie Burks and Jackie Smith, who cornered me in my office one day with a worthy admonition, thank you for affirming possibilities that I was hesitant to own. To David Hutchison, who generously provided time to do what was necessary to move forward, thank you for 
removing what could have been a major stumbling block. To my cohort members, James, Gary, Dana, Glenn, and Janet, thank you for making this shared experience one that I will always treasure. To my dear friends and professional colleagues, thank you for encouraging, checking-for-updates, and generally showing interest. And finally, to my husband, Gene, my children (Wendy, Wesley, and Wallace), my grandchildren (Cooper and Carter), and my parents and siblings, thank you for weathering these past few years with me and for believing that the content, the product, and the process were worth the sacrifice. 


\title{
ABSTRACT \\ THE EFFECT OF TEACHER LEADER INTERACTIONS \\ WITH TEACHERS ON STUDENT ACHIEVEMENT: \\ A PREDICTIVE STUDY
}

\author{
Winifred R. Cohron
}

May 2009

Research literature acknowledges the importance of teacher leadership in school reform initiatives. The literature is replete with qualitative studies describing the experience of teacher leadership in its variety of enactments. The meager amount of existing quantitative data suggests that teacher leadership may have no impact on student learning.

This non-experimental, quantitative study examined the relationship between specific teacher leader interactions with teachers and student achievement. Using an online survey, participants responded to online survey questions about their interactions with teachers around five leadership constructs identified by Lambert (1998) and adapted by the researcher with permission from Dr. Lambert and the Association for Supervision and Curriculum Development (ASCD). The constructs included (a) broad-based and skillful participation in the work of leadership; (b) inquiry-based use of information to inform shared decisions and practice; (c) roles and responsibilities that reflect broad involvement and collaboration; (d) reflective practice/innovation as the norm; and, (e) high student achievement. Student achievement was measured using 
the 2008 School Academic Index of the Kentucky Core Content Test (KCCT). Participants in the study were teachers from Kentucky public elementary schools in the Green River Regional Educational Cooperative (GRREC). The following research question guided the study: What is the relationship between interactions between (a) teacher leaders and teachers, and (b) test scores for schools on the Kentucky Core Content Test (KCCT) after controlling for demographic factors known to negatively impact test scores?

Multiple regression analysis was used to answer the research question. Because the variable correlations using data from all respondents $(\mathrm{N}=567)$ were not strong enough to support a regression analysis, sub-groups of the surveyed participants were tested. Stronger correlations were found between all variables when responses from intermediate (Gr. $4-6)$ teachers $(N=202)$ were tested. The regression analysis was run using only intermediate teacher data. Identification of a teacher leader in the building negatively correlated with student achievement. Interactions related to the student achievement construct was noted as a significant predictor of student achievement as measured by the School Academic Index of the KCCT. 


\section{TABLE OF CONTENTS}

PAGE

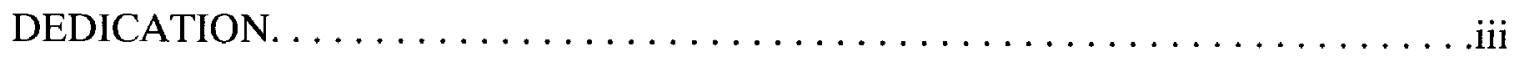
ACKNOWLEDGEMENTS $\ldots \ldots \ldots \ldots \ldots \ldots \ldots \ldots \ldots \ldots \ldots \ldots \ldots \ldots$ iv

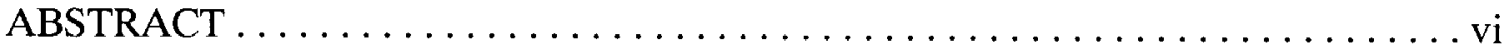

LIST OF TABLES $\ldots \ldots \ldots \ldots \ldots \ldots \ldots \ldots \ldots \ldots \ldots \ldots \ldots \ldots \ldots \ldots \ldots \ldots \ldots$

LIST OF FIGURES $\ldots \ldots \ldots \ldots \ldots \ldots \ldots \ldots \ldots \ldots \ldots \ldots \ldots \ldots \ldots \ldots \ldots \ldots \ldots \ldots$ xiii CHAPTER

I. INTRODUCTION

Introduction $\ldots \ldots \ldots \ldots \ldots \ldots \ldots \ldots \ldots \ldots \ldots \ldots \ldots \ldots$

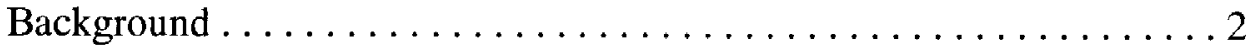

The Problem .................................5

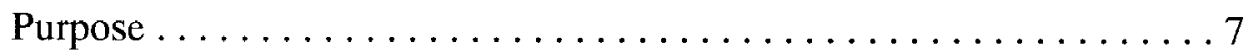

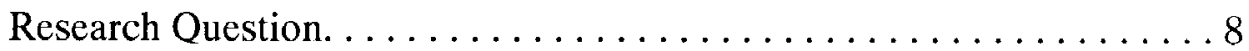

Significance of Study $\ldots \ldots \ldots \ldots \ldots \ldots \ldots \ldots \ldots$

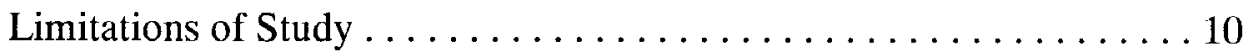

Definition of Terms $\ldots \ldots \ldots \ldots \ldots \ldots \ldots \ldots \ldots \ldots \ldots \ldots \ldots \ldots$

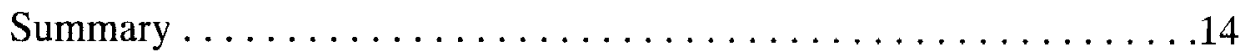

II. REVIEW OF THE LITERATURE

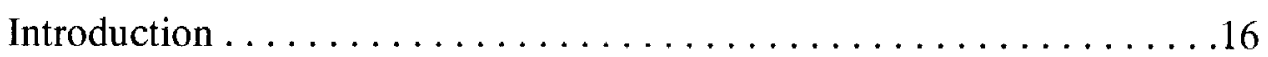

Teacher Leadership Roles . . . . . . . . . . . . . . . . . . . . . . . . 18 
Teacher Leadership and School Leadership Trends . . . . . . . . . . 76

Teacher Leadership and Distributed Leadership . . . . . . . . . . . . . 99

Teacher Leadership and Student Achievement . . . . . . . . . . . 127

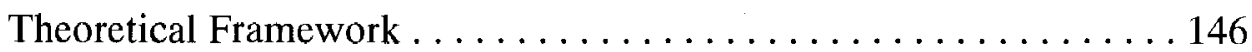

Summary .......................... 151

\section{METHODOLOGY}

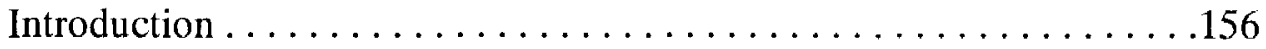

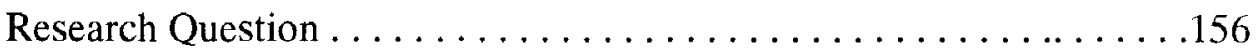

Description of Variables . . . . . . . . . . . . . . . 157

Research Design . . . . . . . . . . . . . . . . . . . . . . . . . 159

Population and Sample . . . . . . . . . . . . . . . . . . . 160

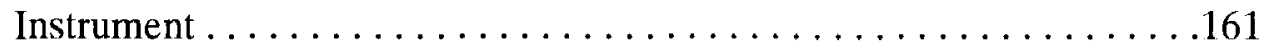

Validity and Reliability ... . . . . . . . . . . 162

Procedure ............................ 164

Data Analysis . . . . . . . . . . . . . . . . . . . . 165

Hypothesis . . . . . . . . . . . . . . . . . . . . 165

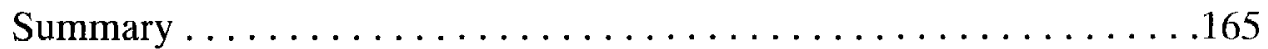

\section{RESULTS}

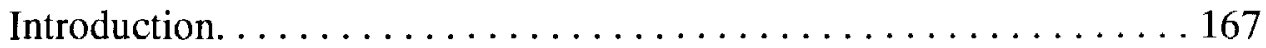

Validity and Reliability. . . . . . . . . . . . . . 168

Procedure. ......................... 170

Descriptive Statistics. . . . . . . . . . . . . . . . . . 172

Research Question. ..........................176 


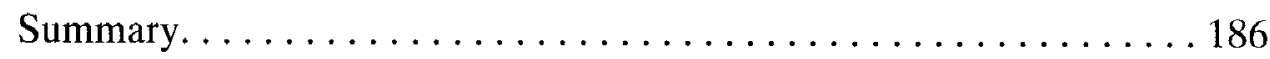

\section{DISCUSSION AND CONCLUSIONS}

Introduction $\ldots \ldots \ldots \ldots \ldots \ldots \ldots \ldots \ldots \ldots \ldots \ldots \ldots \ldots$

Summary of the Study . . . . . . . . . . . . . . . . . . 191

Discussion and Implications. . . . . . . . . . . . . . . . 192

Suggestions for Future Research. . . . . . . . . . . . . . . . 197

Conclusion. ...............................198

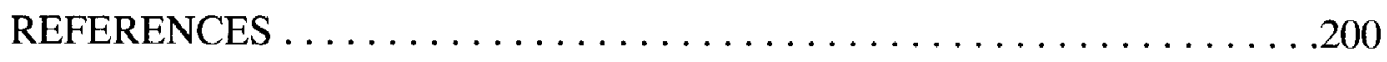

APPENDICES

A. Content Validity Survey $\ldots \ldots \ldots \ldots \ldots \ldots \ldots \ldots \ldots \ldots \ldots \ldots \ldots \ldots$

B. Survey Instrument . . . . . . . . . . . . . . . . . . 219

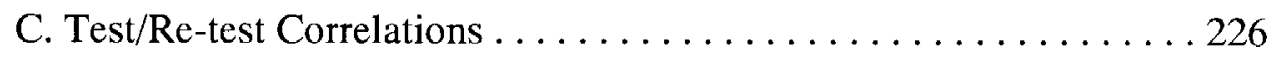

D. Superintendent Permission Forms $\ldots \ldots \ldots \ldots \ldots \ldots \ldots . \ldots 229$

E. Principal E-mail. ......................... 230

F. Expanded School Participation Data.................. 231

G. District Participation Data..................... 235

H. 2008 Academic Indices for Participating Schools ...........237

I. Human Subjects Review . . . . . . . . . . . . . . . 240

J. Survey Instrument Permission . . . . . . . . . . . . . . 242

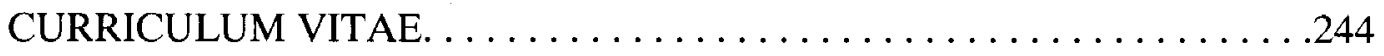




\section{LIST OF TABLES}

TABLE

PAGE

1. Participation Information: Teacher, School, and District. . . . . . . . . . 172

2. Demographic Statistics: Age, Years of Teaching Experience, Years in Current

School.

3. Demographic Information: Gender, Ethnicity, and Education. . . . . . . . . . 174

4. Demographic Information: Grade Level, Teacher Leader in Building. . . . . . . . 175

5. Demographic Information: Subjects Taught. . . . . . . . . . . . 176

6. Correlations for All Variables for All Respondents: Academic Index, \% School African American, \% School Free/Reduced Lunch, Leadership Work, Shared

Decision Making, Collaboration, Reflective Practice, Analysis of Learning, and

Identified Teacher Leader. . . . . . . . . . . . . . . . . . . . . . . 179

7. Correlations for All Variables for 4-6 Grade Teachers: Academic Index, \% School African American, \% School Free/Reduced Lunch, Leadership Work, Shared Decision Making, Collaboration, Reflective Practice, Analysis of Learning and Identified Teacher Leader. . . . . . . . . . . . . . . . . . . 181

8. Descriptive Statistics: Intermediate Teacher Responses to Teacher Leader

Construct.

9. Regression Summary: Teacher Leader Constructs on Student Achievement . . . 183

10. Predictor Variables for School Academic Index . . . . . . . . . . . . . 184 
11. Frequency of Level 4 and Level 5 Responses to Analysis of Learning

Construct Items. . . . . . . . . . . . . . . . . . . . . . . 185 


\section{LIST OF FIGURES}

FIGURE

PAGE

1. Predictive model for teacher leader effect on student achievement $\ldots \ldots \ldots .8$

2. Theoretical framework for analyzing instructional outcomes of participative

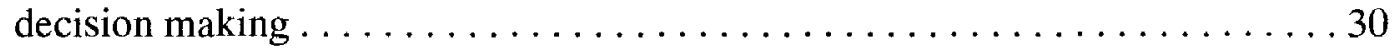

3. Framework for interdisciplinary cross-subject high school leadership

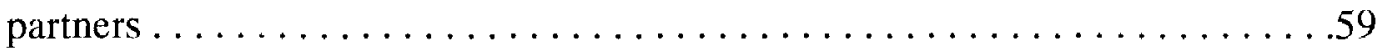

4. A conceptual framework for the impact of teacher-led development work . . . .64

5. Theoretical framework explaining leaders' effects on teachers and their

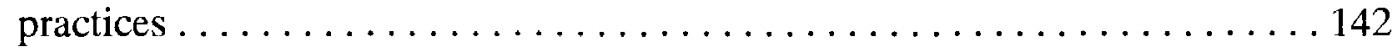

6. Predictive model for research hypotheses $\ldots \ldots \ldots \ldots \ldots \ldots \ldots \ldots \ldots$ 


\title{
CHAPTER I \\ INTRODUCTION
}

\author{
Introduction
}

Since the release of $A$ Nation at Risk (National Commission on Excellence in Education) in 1983, educational professionals, federal and state governing bodies, and the general public have been concerned about school improvement, defined as high levels of achievement for all students. The focus of blame and the mandate to change centered on school leadership. Researchers gathered data to clarify the characteristics of effective leaders in effective schools, learning about "the centrality of instructionally focused leadership" (Murphy, 2005, p. vii). Successful principals appeared "strongly focused on leading teachers and improving instruction in the classroom... and were more likely to use a variety of instructional leadership practices" (LaPointe \& Davis, 2006, p. 34). These instructional leadership practices required a broader understanding of school leadership, "one that shifts from a single person, role-oriented view to a view of leadership as an organizational property shared among administrators, teachers, and perhaps others" (Smylie \& Hart, 1999, p. 428).

Teacher leadership created opportunities to "increase professionalism, redistribute authority, and increase collegial interaction" (Keedy, 1999, p. 787). As a form of distributed leadership, teacher leadership promoted broader contribution to "school 
reform or student learning by influencing others to improve their professional practice, or [by] identifying and contributing to a community of leaders" (Moller \& Katzenmeyer, 1996, p. 5). As a source for improving student learning, teacher leaders can be "a strong catalyst for ...change. By using the energy of teacher leaders as agents of school change, the reform of public education will stand a better chance of building momentum" (Moller \& Katzenmeyer, p. 2).

\section{Background}

Teacher leadership has evolved through a variety of role descriptions. Beginning in the 1980's, education reform called for increased involvement of teachers in decisions regarding all aspects of school (Smylie, 1992a). Increased teacher participation in decision making was intended to improve the effectiveness of decisions on student learning (White, 1992) as well as teacher motivation (Smylie). While some teachers did experience improved attitudes toward work as a result of involvement in decision making for school-wide policy (White), many more did not. Teachers who chose to assume more responsibility for school improvement frequently ran up against the cultural norm of individual teacher autonomy (Smylie). Colleagues' resistance to change made some teachers reluctant to become caught between their peers and administrators (Cameron, 2005; Griffin, 1995; Smylie). In addition, teacher involvement in school-based decision making did not result in changes in instructional practice across schools. The belief that student learning would be positively impacted by teacher participation in school decisionmaking proved false (Smylie, Lazarus, \& Brownlee-Conyers, 1996; Stone, Horejs, \& Lomas, 1997; Taylor \& Bogotch, 1994).

Teacher leadership was commonly understood as what teachers did outside the 
classroom in addition to full-time teaching (York-Barr \& Duke, 2004). However, some researchers found that teachers' influence on students was the focus of teacher leadership (Cheng, 1994; Katyal \& Evers, 2005). Outside influence on other teachers and school decisions were usually offset by additional work load, less personal time, and loss of undivided attention to the individual teacher leader's students. Regardless, classroom teacher leaders viewed work with other colleagues outside the classroom as important for the growth of their students and their school (Cruz, 2003; Katyal \& Evers; Middlebrooks, 2004, Ovando, 1996; Silva, Gimbert, \& Nolan, 2000).

Other studies suggested that teachers must be involved in leadership for teaching and learning if school improvement was to occur (York-Barr \& Duke, 2004). Teacher leaders came to be seen as instructional change agents through transparency of their attitudes and practice (Beattie, 2002; Hatch, White, \& Faigenbaum, 2005) as well as their interpersonal capacity (Frost \& Durrant, 2002). Teacher leaders' coaching of other teachers appeared to facilitate instructional change (Kohler \& Crilley, 1997) for teachercolleagues. Teacher leaders exercised influence over other teachers' instructional practices when the teacher leader was perceived as possessing cultural, human, and social capital. Cultural capital designated the style of interaction with others. Human capital referred to skills, knowledge, and expertise. Social capital applied to trusting relationships and networks. (Spillane, Hallet, \& Diamond, 2003).

Principals also played "a major role in teacher work design" (Whitaker, 1997, p. 5) due to their responsibility for the "development of leadership capacity" (Lambert, 2003b, p. 53) within the school. Ambiguities and uncertainties about teacher leader roles required forging new norms of behavior for both principals and teacher leaders (Smylie 
\& Brownlee-Conyers, 1992). "Because most new teacher leadership roles depend heavily on teacher leader-principal interaction and collaboration, principals are in first-order positions to block, to support and facilitate, and to shape the nature and function of teacher leadership in their schools" (p. 151). The attention and support of principals significantly contributed to the importance with which teachers viewed those in teacher leader positions (Hart, 1994). Conversely, because school leadership has become more reliant on task competency rather than formal position, principals find themselves more dependent upon the vast competencies of teachers within their schools (Brown \& Anfara, 2002). Principals have been encouraged to share leadership with teachers in order to improve teaching and learning, as well as create a climate that supports emerging teacher leaders (Yarger \& Lee, 1994).

Distributed leadership theory supported the need for principals to share leadership with teachers (Gronn, 2002; Muijs \& Harris, 2003). The emphasis of teacher leadership upon collaborative action and empowerment of all toward accomplishment of a shared goal reflected distributed leadership theory (Muijs \& Harris). Redundant leadership roles for change, involving many individuals with different positions in schools acting as change leaders, provided mutual reinforcement of desired change and assured attention to critical tasks (Heller \& Firestone, 1995). Furthermore, some data led researchers to believe that one teacher leader per school was not enough to bring teachers and administrators together in shared leadership (Datnow \& Castellano, 2001).

Distributed leadership research moved toward studies of school-wide leadership teams whose purpose was to improve student achievement. This evolution into teacher leadership teams raised new questions about how such a configuration would fit into 
existing organizational structures, rules, and relationships, and how effective such groups would be in improving teaching and learning (Chrispeels \& Martin, 2002). Emphasis on teacher leader teams which encouraged and facilitated job-embedded professional development determined by teachers was believed to contribute to a culture of learning for teachers and students (Thompson, Gregg, \& Niska, 2004). In one study, results suggested that when teacher leader teams in different schools used similar, effective data analysis strategies, teachers' understanding of students' learning needs improved (Temperley, 2005).

Interestingly, while teacher leadership, either by individuals or teams, has been assumed to support improved student performance, the direct link between teacher leadership and student achievement remains unclear (Harris, 2005). Teacher leadership has not been shown to have a significant effect on student engagement (Leithwood \& Jantzi, 1999, 2000) or student outcomes (Silins \& Mulford, 2004), neither of which have been defined in a way to connote student achievement. The question regarding the effect of teacher leadership on student achievement remains unanswered.

The Problem

Teacher leadership has been described as an avenue to school improvement, a pathway toward reform of the public education system. As a re-tooling of teachers' roles, teacher leadership "is concerned with teachers helping teachers so that teachers can, in turn, better help students" (Pellicer \& Anderson, 1995, p. 22). Teachers enact leadership "when they are contributing to school reform or student learning (beyond the classroom), influencing others to improve their professional practice, or identifying with and contributing to a community of leaders" (Moller \& Katzenmeyer, 1996, p. 5). 
While these behaviors have always been informally demonstrated by teachers as they taught in their individual classrooms (Crowther \& Olsen, 1997), research about teacher leadership in the 1980's and 1990's inspired action. In many districts and schools, new full- and part-lime jobs were created for school-level teacher leaders, "positions that capitalized on teacher instructional knowledge" (Silva et al., 2000, p. 780). Job descriptions varied among districts, and among schools within districts. The one constant was the expectation that the teacher leader, regardless of title or job description, would work more directly with teachers, and frequently students, to improve student achievement.

Many Kentucky schools welcomed the additional assistance a building-level teacher leader could offer the school principal. With the passage of the Kentucky Education Reform Act of 1990, annual high-stakes testing accountability became reality for all Kentucky schools. District and school leaders recognized the need to support instructional improvement and believed that building-level teacher leaders would enhance the efforts of already overworked principals. The position became normative for many Kentucky schools. "States and local jurisdictions increasingly recognize teacher leadership as a strong and pervasive trend" (Clemson-Ingram \& Fessler, 1997, p. 104).

Although full-time building level teacher leaders became fixtures in many Kentucky schools, no research has measured the impact of this relatively new position on student achievement. This concern does not belong solely to Kentucky. "In the era of the most stringent accountability in the history of education, the near absence of attention to accountability issues in the teacher leadership literature is difficult to justify" (Murphy, 2005). Improved student achievement as a result of improved instruction was the desired 
outcome of teacher leader positions. Minimal data to support or refute achievement of that goal currently exists. One possible reason for the lack of data could be that educational leaders avoided asking whether investing in teacher leader positions actually improved student achievement. An appropriate methodology for measuring teacher leader outcomes evaded researchers, and were, at best, unclear and fraught with complexity.

\section{Purpose}

While the research literature acknowledges the importance of teacher leadership in school reform initiatives, "research concerning the relationship of teacher leadership to student learning is...equivocal (Smylie, 1996, p. 576). The purpose of this study will be to measure the relationship between teacher leader interactions with teachers and student achievement data as measured by the Kentucky Core Content Test (KCCT). No studies have attempted to measure this relationship although teacher leader positions have existed in many Kentucky schools for the past decade. The KCCT is the state assessment used to measure student achievement for students who attend Kentucky public schools. All Kentucky schools have received the mandate from the state legislature requiring students to score 100 on a 140 point accountability index scale by 2014 . Teacher leaders have been entrusted with an important role in moving teachers and students toward this goal. Measuring the efficacy of teacher leaders' interactions with teachers toward improved student performance could inform practice and policy.

A theoretical model of leadership capacity development formulated by Dr. Linda Lambert (1998) and derived from literature on school reform will shape the study. Lambert's framework includes five constructs which can be applied to the interactions between teacher leaders and teachers. One critical element in the framework is high 
student achievement, which refers to successful student performance of tasks that have a real-world relationship to work or the general society. Data leading to high levels of student achievement come from multiple types and forms of assessments at various points in time, and is used as evidence to determine who needs specific interventions to master the content. The goal of achievement data is to support continuous improvement for all students. This is the purpose of all school leadership. For the purpose of this study, it is the primary goal of interactions between teacher leaders and teachers. In this study, Lambert's student achievement construct will be referred to as analysis of learning.

\section{Research Question}

The purpose of this study is to measure the relationship between teacher leader interactions with teachers and student achievement as measured by the KCCT, while controlling for demographic factors known to negatively impact student test scores. The study will be predictive as shown in Figure 1: (a) interactions between teacher leaders and teachers lead to (b) effective classroom instruction by teachers which leads to (c) greater acquisition of academic knowledge and skills by students which leads to (d) higher student achievement and overall school scores on the KCCT.

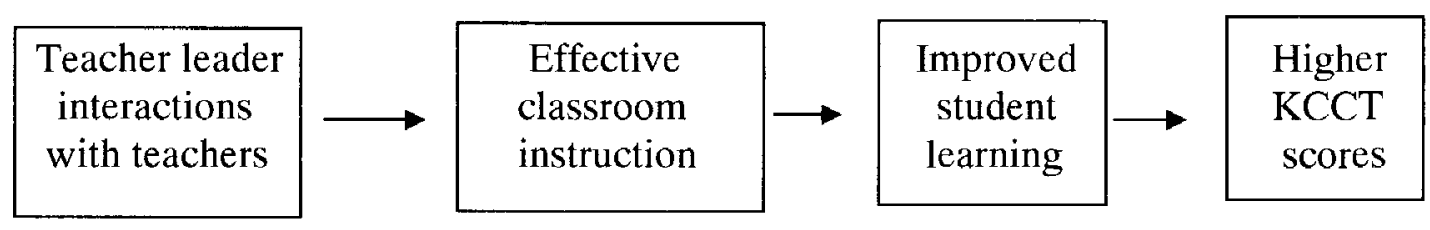

Figure 1. Predictive model for teacher leader effect on student achievement.

The specific research questions guiding the study seek to determine the following: What is the relationship between interactions between (a) teacher leaders and teachers and (b) test scores for schools on the Kentucky Core Content Test (KCCT), after 
controlling for demographic factors known to negatively impact test scores?

Significance of Study

Research on teacher leadership continues to be an important focus for the advancement of school reform. Due to increased demands and increased complexities, school leadership in the $21^{\text {st }}$ century requires that many individuals participate in leadership functions (York-Barr \& Duke, 2004). While teacher leadership is believed to maximize the skills of in-house experts for school improvement (Whitaker, 1997), "documenting the ways this reform functions and the outcomes it produces" (Murphy, 2005, p. 164) remains a critical area for teacher leader research. Results showing that teacher leadership "makes schools richer or more productive for students" (Schmoker \& Wilson, 1994, p. 148) are minimal. Few districts have scrutinized their investment in teacher leader positions to determine if the results were improved student achievement. While this omission is understandable due to the challenge of determining how to accurately measure such a multifaceted construct, more studies are needed to arrive at definitive conclusions about the effectiveness of teacher leadership.

This study will attempt to build upon and add to the research literature by asking if specific interactions between teacher leaders and teachers predict student achievement. This information could be significant to teacher leaders, principals, professional development directors, district administrators, state education department leaders, policy makers, and teacher leader preparation program designers. If specific interactions point to higher school KCCT Academic Indices, all of the previously mentioned groups would want to know in order to embed those behaviors into training programs and regular practice. Teacher leader positions could become a standard school position and teacher 
leader job descriptions could be configured to focus on the specific behaviors known to predict higher levels of student achievement.

The study is also significant because of the type and level of data gathering. Teachers will be asked to respond to a questionnaire which will describe specific types of activities in which they might engage with teacher leaders. Previous teacher leader research has tended to be qualitative in nature, recounting the experiences of teacher leaders in order to describe the phenomenon of teacher leadership. Many of these studies naturally sought teacher leaders as participants rather than the teachers who were the recipients of teacher leader services. Teachers have frequently "not been included in the current discussion about teacher leadership" (Wasley, 1991, p. 161). This study will use quantitative measures to analyze data drawn from teacher responses to survey questions about their interactions with teacher leaders.

\section{Limitations of Study}

The annual school Academic Index from the Kentucky Core Content Test (KCCT) used as the measure of student achievement in this study is specific to Kentucky. This achievement data may not be comparable to student results from assessments used by other states. In this study, each school's Academic Index for 2008 will be paired with teacher leader interactions with teachers during the 2007-2008 academic year. Tracking teacher leader behaviors and student achievement over a longer period of time would yield more stable results, more accurately predicting which specific behaviors could be connected to higher levels of student achievement.

Other limitations will also characterize this study. The most prominent limitation will be the use of a predictive rather than a causal model. While a causal model would be 
more desirable to pinpoint specific causes (teacher leader behaviors) and effects (student achievement), a direct link is not possible due to intervening variables, i.e. effective classroom instruction and improved student learning, which cannot be directly measured. A predictive model was selected because it will yield "a linear equation that identifies the best weighted combination of independent variables in the study to optimally predict the [dependent] variable" (Meyers, Gamst, \& Guarino, 2006, p. 149). Notwithstanding, a predictive model will not yield the same level of certainty as a causal model.

Second, only elementary school teachers in one region of Kentucky will be included in the sample. Middle and high schools in the same region could yield different results. Limiting the sample to one region of Kentucky and to only elementary schools in that region could limit generalizability across school levels throughout the state.

Third, teacher participants will be asked to complete the survey online. While providing easier and less costly access to participants, conditions under which surveys will be completed will be out of the control of the researcher. Teachers may be less likely to complete an online survey than one administered during a faculty meeting. Interpretation of directions could differ among participants, skewing responses.

\section{Definitions of Terms}

Operational definitions of terms to be used in the study are listed in this section. Academic Index for Elementary Schools (AI): The Academic Index is derived from academic content values in Reading (22\%), Mathematics (22\%), Science (14.5\%), Social Studies (14.5\%), Writing on Demand (7.25\%), Writing Portfolio (7.25\%), Arts and Humanities (5\%), Practical Living and Vocational Studies (5\%) resulting from student scores on the Kentucky Core Content Test (KDE, 2008a). 
Analysis of learning: Lambert's (1998) high student achievement explained as successful performance of tasks which are "based on real tasks that have a relationship to work and life in society or in the family" (Lambert p. 23). In this study, analysis of learning will be used for Lambert's high student achievement construct.

Commonwealth Accountability Testing System (CATS): The total program designed to monitor improvement in student learning. The CATS includes the Kentucky Core Content Test (KCCT), writing portfolios and prompts, alternate assessments for students with severe to profound disabilities, the ACT, PLAN, and non-academic components (KDE, 2008b).

Core Content for Assessment: The content and skills identified as essential for all students to know and be able to apply. The Core Content for Assessment provides the parameters for development of the Kentucky Core Content Test (KDE, 2008c).

Elementary School: School which serves students from the Primary Level, beginning with kindergarten, through a minimum of the Intermediate Level, usually Grade Five (KDE, 2008d).

Green River Regional Educational Cooperative (GRREC): One of eight regional groups in Kentucky which offer assistance and expertise for the benefit of their member school districts. GRREC provides comprehensive educational services and programs that support member districts and their schools in school improvement efforts (KDE, 2008e).

Kentucky Core Content Test (KCCT): The KCCT is the most important component of the Commonwealth Accountability Testing System (CATS) because of its contribution to a school's Academic Index. The KCCT measures student mastery of the Kentucky Core Content for Assessment as well as higher order thinking and 
communication skills. Composed of both open response items and multiple choice questions, the KCCT is administered each spring to Kentucky public school students. All students in Grades Three through Eight take Reading and Math tests. Students in Grades Four and Seven also take tests in Science, Practical Living / Vocational Studies, and complete a Writing Portfolio. Students in Grades Five and Eight take tests in Social Studies, Arts and Humanities, and complete a response to a Writing-on-Demand prompt. High school students also participate in the KCCT. Tenth grade students are assessed in Reading and Practical Living/Vocational Studies, and the PLAN (part of the Educational Planning and Assessment System). Eleventh grade students take tests in Math, Science, Social Studies, Arts \& Humanities, as well as the ACT (part of the Educational Planning and Assessment System). Twelfth grade students complete a Writing Portfolio and respond to a Writing-on-Demand prompt during the fall of their senior year (KDE, 2008f).

Kentucky Performance Report (KPR): The KPR gives detailed information about the results of the KCCT and other components of CATS for each school and district (KDE, 2008g).

Teacher leader: A certified teacher who leads and engages in some or all of the following: (a) shared decision making, (b) collaboration to improve teaching and learning, (c) processes that support school improvement, (d) individual and collegial professional learning, and (e) advocacy to affect change (Muijs \& Harris, 2006). A teacher leader may be a classroom teacher who performs these functions in addition to teaching or may be employed as a full-time teacher leader. 


\section{Summary}

Teacher leadership has been a topic of school reform research for the past two decades. Studies have examined teacher leadership through a variety of lenses ranging from the wider view of "assuming greater leadership of the organizations in which they work" (Murphy, 2005, p. 3) to a narrower focus of "influencing and engaging colleagues toward improved practice" (Wasley, 1992, p. 21) within a school. While some results assert "the central place of teacher leadership in the school improvement plan has been identified" (Murphy, 2005, p. vii), others would caution that the direct link between teacher leadership and student achievement remains unclear (Harris, 2005; Leithwood \& Jantzi, 1999, 2000; Silins \& Mulford, 2004).

Kentucky public schools have invested heavily in developing teacher leaders. Many districts and schools have created full-time teacher leader positions to support the instructional leadership work of the building principal. Leadership teams made up of fulltime teachers are becoming the rule rather than the exception. Some Kentucky colleges and universities are re-designing graduate programs to include a teacher leader endorsement which would be recognized by the Kentucky Educational Professional Standards Board. The lack of recognized teacher leader standards and guidelines exacerbates the re-design process. Moreover, the current dearth of teacher leader training programs in Kentucky colleges and universities indicates a dissonance between common practice and substantiating data.

While a broad swath of research speaks to the wisdom of these initiatives, the concern remains that minimal data suggest improved student achievement as a result of teacher leadership. This study will involve a quantitative analysis of the relationship 
between teacher leader interactions with teachers and 2008 KCCT scores. Elementary teachers from 124 schools in one region of Kentucky will be asked to answer survey questions regarding interactions with the building-level teacher leader(s). Survey results and $2008 \mathrm{KCCT}$ school academic indices will be analyzed using a predictive model. The study is intended to advance the knowledge about the connection between teacher leadership and student achievement. 


\section{CHAPTER II \\ REVIEW OF THE LITERATURE}

\section{Introduction}

This study addresses the relationship between teacher leader interactions with teachers and student achievement. The literature regarding teacher leadership traces the evolution of this role through that of the classroom teacher participating in school-based decision making to a full- or part-time managerial position, i.e., department heads, quasiadministrative positions outside the classroom, classroom teacher, and finally to participation in distributed leadership functions (Silva, Gimbert, \& Nolan, 2000; Katyal \& Evers, 2005).

Research on teacher leadership continues to be an important focus for the advancement of school reform. Due to increased demands and increased complexities, school leadership in the 21 st century requires that many individuals participate in leadership functions (York-Barr \& Duke, 2004). Herzberg's Motivation Theory (Herzberg, Mausner, \& Bloch, 1959) supports the concept that schools provide responsibility, achievement, recognition, advancement, and growth opportunities to teachers which are intrinsic to the work of teaching. Teacher leadership amplifies the professional reputation of teachers and maximizes the skills of in-house experts for school improvement initiatives (Whitaker, 1997). Teacher leadership defines the processes through which teachers influence the school community to improve 
instructional practice for the purpose of enhancing student learning and increasing student achievement (York-Barr \& Duke, 2004).

Teacher leadership is primarily concerned with developing high-quality learning and teaching in schools. It has as its core a focus on improving learning and is a mode of leadership premised on the principles of professional collaboration, development, and growth. Teacher leadership is not a formal role, responsibility or set of tasks. It is more a form of agency where teachers are empowered to lead development work that impacts directly on the quality of teaching and learning. Teacher leaders lead within and beyond the classroom. They identify with and contribute to a community of teachers and influence others towards improved educational practice (Lambert \& Harris, 2003, p. 43).

This literature review focuses on the evolution of leadership roles for teachers and the impact of these leadership roles on collegial interactions for improved teaching and learning. This chapter identifies overall themes and trends in teacher leadership research conducted since 1990. Sections are included regarding a) teacher leadership roles, b) teacher leadership and school leadership trends, c) teacher leadership and student achievement, d) a theoretical framework for the study, and e) a summary of findings.

Research procedures consisted of searching online university databases (EBSCO Host, EBSCO Academic Premier, and ProQuest) to obtain references of peer-reviewed scholarly studies related to the topic of teacher leadership. There was purposeful omission of teacher leadership studies pre-dating those reviewed in this chapter, i.e., those published before 1990, with the exception of several seminal studies. The purpose was twofold: (a) to examine teacher leadership from a post-reform perspective, i.e., 7 years after the release of A Nation at Risk (National Commission on Excellence in Education, 1983) and post-Kentucky Education Reform Act (KERA, 1990); and (b) to examine the most current literature available to determine what remains untested and unknown about the effects of teacher leadership on student achievement. The majority of 
the literature review is descriptive rather than empirical. This is due to the nature of the studies conducted about teacher leadership during the past 17 years. Numerous qualitative studies readily illustrate the experience of teacher leaders, while fewer quantitative studies wrestle with the influence of teacher leadership on student achievement.

\section{Teacher Leadership Roles}

Teacher leaders envision themselves as facilitators of improving instructional practices within their schools. Teacher colleagues, however, may not share the same perception (Smylie \& Denny, 1990). As school reform initiatives have evolved since the publication of A Nation at Risk (National Commission on Excellence in Education, 1983), so have the insights about the nature and role of teacher leaders in the implementation of distributed leadership. Teacher leaders serve in a variety of formal and informal positions in the daily operation of schools. Formal positions may include department head, curriculum specialist, mentor, or member of a school-based management team. Informal roles may include peer coach, facilitator of teacher-studygroups, or modeler of best instructional practices. Some teacher leaders assume full-time leadership positions while others maintain full-time jobs as classroom teachers while assuming other leadership responsibilities (York-Barr \& Duke, 2004). The studies addressed in this section examine the role of teacher leaders in school-wide decisionmaking, in classroom teaching, and as instructional change agents. Teacher leaders' perceptions of their functions and how these functions depend upon principal leadership are also reviewed. 


\section{Teacher Leaders as School-Based Decision-Makers}

One of the first waves of educational reform in the 1980 's called for increased involvement of teachers and parents in decisions impacting all elements of school life (Smylie, 1992a). Teacher involvement in this aspect of school life, previously reserved to school administrators, posed new opportunities and new challenges. One of the purposes of increased teacher participation in decision making was to use teacher expertise to improve both the quality of decisions to meet student needs (White, 1992) and to improve teacher motivation in executing decisions (Smylie, 1992a).

Smylie (1992a) tested the influences of different organizational and psychological factors on teachers' willingness to participate in decision making at the school level. The researcher used the literature to determine the factors which would provide a framework for analysis of teachers' willingness to participate in different areas of decision making. The independent variables in the study included (a) the principal-teacher working relationship, (b) norms influencing work relationships among teachers, (c) teachers' perceived capacity to contribute to or make decisions, and (d) teachers' sense of responsibility and accountability in their work with students. The dependent variable was teacher willingness to participate in decision making in areas of (a) personnel, (b) curriculum and instruction, (c) staff development, and (d) general administration.

The sample for the Smylie (1992a) study consisted of 200 teachers in a midwestern metropolitan K-8 school district. The board of education and the teachers' association of the district agreed on a contract which stipulated the establishment of school councils in each school. The purpose of the school councils was to give teachers meaningful opportunities to participate in school-level decision making. School councils 
had the authority to make decisions about organizational, administrative, and instructional issues, as well as all other professional matters which dealt with district goals. While teachers were not required to participate as council members, each school's principal and faculty were mandated to determine council membership structure.

The instrument Smylie (1992a) used for data collection was a survey distributed in faculty meetings and by mail to the district's 200 teachers during the first year of school council implementation. The response rate was $60 \%(N=116)$. The survey used a 4-point Likert-type scale with anchors ranging from $1=$ not willing to $4=$ very willing. Survey items represented areas of decision making in which teachers were willing to participate: (a) personnel, (b) curriculum and instruction, (c) staff development, and (d) general administration. Internal reliability coefficients for scale scores ranged from 0.6001 for personnel decisions to 0.9068 for staff development decisions. Five variables defined the principal-teacher working relationship: (a) teacher participation in school decision making, (b) openness of expression, (c) principal's emphasis of school goals, (d) autonomy at work, and (5) principal's facilitation of teachers' work. Four variables defined teacher's working relationships with other teachers: (a) professional equality, (b) professional autonomy, (c) professional privacy, and (d) opposition to peer judgment. Two variables defined teachers' perceived capacity to contribute to decisions: (a) professional outcome expectancy and (b) certainty of teaching ability. Professional outcome expectancy meant teachers' knowledge about the relationships between instructional strategies and student outcomes. Certainty of teaching ability meant teachers' perceptions of their own ability to achieve desired student outcomes. Two variables described teachers' sense of responsibility and accountability: (a) teachers' 
responsibility for student learning, and (b) teachers' belief that they should be held accountable for their work with students. Survey items representing these variables came from previous studies of social organization of schools and teachers' working relationships with administrators and other teachers. Internal reliability coefficients ranged from 0.8191 for teacher participation in decision making to 0.6652 for autonomy at work. Factor analysis tested for possible multi-collinearity. Comparison of mean scores assessed differences in teachers' willingness to participate in different areas of decision making. The researchers used multiple regression to analyze the relationships between teachers' willingness to participate in decision making and each set of independent variables.

Findings of the Smylie (1992a) study indicate that teachers' relationships with their principals significantly influenced teachers' willingness to participate in all areas of school decision making. The norm of professional privacy provided a slightly significant negative impact on teachers' willingness to participate in decision making about curriculum and instruction as well as staff development. A combination of factors influenced teachers' willingness to participate in personnel decisions: (a) principalteacher relationship, (b) responsibility for student learning, and (c) teacher accountability. Opposition to peer judgment indicated a significantly negative predictor of teachers" willingness to participate in personnel decisions. Smylie (1992a) noted that opposition to peer judgment is a pivotal norm that defines teachers' relationships with colleagues and could mediate the influence of their sense of responsibility and accountability for work with students on their willingness to make decisions about personnel.

While Smylie (1992a) noted that study results were consistent with other research, 
study limitations consisted of the context of a single school district and a specific contractual agreement. However, the findings indicate that simply having established policies and procedures for teacher participation in decision making does not make willing and meaningful participation occur. Teacher participation in decision making would require individual and organizational change that cannot be legislated.

Professional learning opportunities for principals and teachers regarding work roles and working relationships that support participatory decision making are necessary prerequisites. Teachers must be able to reconcile social and normative conflicts with colleagues in order to participate effectively in school decision making.

Smylie (1992a) suggested that future research address other factors that may influence teachers' willingness to participate in school decision making. Studies could identify relationships teachers perceive between these factors, as well as the relative importance of each one. More comprehensive studies could allow generalizability across schools and school districts.

White (1992) chose a different aspect of teacher involvement in school-based decision making to study, examining the kinds of decisions in which teachers were involved as a result of school decentralization. White (1992) studied the extent to which teachers believed that the changes caused by decentralization influenced their authority to affect school policy and enabled them to better meet student needs. The researcher used stratified random sampling to select study participants. Initial selection criteria included districts which had practiced decentralization of authority for at least 5 years; districts with high decentralization of budget, curriculum, and staff decisions; and districts with student enrollments of no more than 22,000 students. From these districts, the researchers 
selected 10 teachers from three elementary schools $(n=30)$ from three different districts in Montana, California, and Minnesota. Respondents also included principals and central office administrators from each school district.

White (1992) conducted semi-structured interviews with each participant and then analyzed the data for emerging themes related to the research purpose. Results of the study indicated that teachers with the highest degree of involvement in school decision making saw the greatest benefits of decentralization. The author surmised that a greater degree of involvement of all teachers in decision making would result in more widespread perception of benefit. However, the respondents indicated that flaws existed in the decentralization initiative. Lack of financial support for teacher training on the shared decision-making process and administrators who demonstrated reluctance to share authority and encourage teacher input emerged as the two most prominent barriers. A third roadblock named was the overall hierarchical structure of the school organization. Participants reported that teachers remained limited by traditional patterns of top-down authority structures.

Despite these shortcomings, the researcher noted three findings which indicated the symbolic importance of decentralization of school decision making. First, increased decision making authority raised teachers' interest in teaching. Second, increased decision making authority reduced teacher isolation. Third, increased teacher decision making authority improved teachers' sense of self-esteem about their jobs and their personal capabilities. While the ultimate motivation behind increased teacher participation in school decision making was improvement in student learning, the author could only imply that result. White (1992) concluded that when teachers felt better about 
their work environment and became more interested in developing new and improved instructional strategies that greater student learning would occur. The author additionally surmised that an increase in teacher self-esteem might attract students to the teaching profession.

Taylor and Bogotch (1994) extended the research about teacher leadership roles by studying the effects of teacher participation in school decision making on teacher and student outcomes. The premise behind the study was that teacher participation in school decision making should improve outcomes for teachers and students. Teacher participation referred to teacher involvement in making decisions about issues which impacted their activities or job assignments. Teacher outcomes included job satisfaction and teacher attendance. Student outcomes consisted of student achievement, behavior, and attendance. The independent variable was teacher participation in school decision making. The dependent variables were teacher and student outcomes. The study occurred in a large, diverse urban district with 300,000 students in 250 schools. The district initiated a restructuring program to increase teachers' participation in school-level decision making. The sample of schools selected for the study included elementary and senior high schools chosen from two groups of schools in the district. The first group consisted of schools that piloted the district school-level decision-making initiative. The second group included non-pilot schools that matched the pilot schools in organizational and demographic characteristics in order to compare schools. A total of 33 schools from both groups participated in the study, 16 from the pilot group and 17 from the non-pilot group.

Data collection consisted of administration of a questionnaire developed by 
previous researchers to measure teachers' involvement in 19 decision items. Respondents indicated the extent to which they actually participated in decision making for each item and the extent to which they desired participation. No anchors were given. Discrepancy scores of this instrument showed Cronbach's alpha reliability coefficients ranging from 0.66 to 0.83 . The response rate was a low $39 \%$, a limitation of the study. However, Taylor and Bogotch (1994) used the research of Wunsch (1986) to determine that this rate reflected the surveyed population with $95 \%( \pm 3 \%)$ confidence. Additionally the demographic profile of the respondents reasonably matched each school-wide profile, allowing confidence that the sample was representative of the population.

Taylor \& Bogotch (1994) also administered the Job Descriptive Index Scale (JDI), one of the most widely used measures of job satisfaction. Job satisfaction referred to feelings teachers had about their jobs. Survey anchors were not indicated. The 300 teachers who responded to the participation questionnaire received the JDI and returned these surveys at a rate of $71 \%$. The power analysis conducted before survey administration indicated that a sample of 120 participants would provide a power level of 0.70 , with an effect size of 0.30 when alpha $=0.05$. The number of respondents exceeded the estimate required by the power analysis. Data on teacher and student attendance, student achievement, and student behavior came from records provided by the district office. These variables were reported as gain/loss scores, computed by subtracting data for the school year prior to the teacher participation program from data representing the third year of program implementation. Pearson correlations determined the strength of the gain/loss scores and yielded significant correlations above $r=0.80$ for each variable except teacher attendance. The mathematics portion of the Stanford Achievement Test 
provided gain/loss scores to gauge student achievement. Teacher and student attendance was measured by the percentage of days attended during the school year. Student behavior was calculated as the percentage of students who were suspended during the school year.

The researchers used factor analysis of actual teacher participation data to identify the dimensions of teacher participation in decision making. Four factors emerged, including associated technology, managerial, instructional materials, and core technology. Associated technology included student rights, standardized testing policy, student discipline codes, student achievement reporting, grading policies, teacher's performance evaluation, staff development and students' assignment to classes. The managerial factor referred to budget development, spending priorities, staff hiring and teacher assignment, facility design, and student removal for special instruction. The instructional materials factor included which texts and workbooks were available and which were used. The final core technology factor included how to teach, what to teach, and the teacher's subject/grade assignment. Cronbach's alpha coefficients for each of the four factors were $.84, .78, .89$, and .66 , respectively. These four factors accounted for $57 \%$ of the variance in items involving teacher participation.

Teacher participation factors were then correlated to teacher and student outcome variables. A significant correlation occurred between teacher participation in decisions related to core technology and student attendance $(r=.43, p \leq .05)$, with a strong effect size of $18 \%$. There were no significant correlations between any teacher participation factor and student achievement, student behavior, or teacher attendance.

A second correlation between teacher participation factors and the Job 
Descriptive Index Scale revealed a consistent pattern about the relationship between teacher job satisfaction and involvement in specific areas of decision making, even though the largest effect size was a weak $6 \%$. These overall weak correlations caused Taylor and Bogotch (1994) to question the usefulness of teacher participation in decision making to achieve the desired outcomes that the district re-structuring model intended. A multivariate analysis of variance (MANOVA) procedure calculated the differences between the two participating school groups regarding teacher and student outcome variables and the Job Descriptive Index Scale. MANOVA results indicated no significant mean differences between outcome variables for both participating school groups. Taylor and Bogotch interpreted these results to mean that teacher participation in school decision making produced no measurable impact on student achievement, student behavior, student attendance or teacher attendance. The researchers noted that collegiality among teachers and between teachers and administrators was not evident, and that teachers who participated in decision making did not subsequently change instructional practice in order to impact student learning.

While Taylor \& Bogotch (1994) did not uncover any significant relationship between teacher participation in school decision making and student achievement, research on the impact of teacher involvement in school governance continued. Griffin (1995) used informal qualitative case study methodology to examine what teachers believed to be classroom-level consequences of their schools' efforts to re-design the role of teachers for increased involvement in school decision making. Descriptions of these efforts included site-based decision making, career ladder programs, design of horizontal school organizational structures, and creation of formal teacher leadership positions. A 
convenience sample of five teachers known to the researcher participated in the study. Each teacher worked in a different elementary school in separate school districts. The five schools ranged from large, urban, multi-ethnic and multi-lingual student populations, to affluent suburban students, and beyond to small rural community schools. The participants were veteran teachers with 7 to 17 years' experience who taught at an array of grade levels. Data collection involved recording of monthly conversations between the researcher and the individual interviewees over an approximate 3 -year period. The researcher also posed direct questions to elicit specific information. Data analysis consisted of an examination of conversations for common themes throughout the five teachers' accounts. The researcher shared analysis results with each participant, who then offered suggestions for revision.

Study results indicated that re-design for increased teacher involvement in school decision making primarily addressed school-wide issues. Those issues included evaluating student achievement practices, aligning district and school curriculum requirements, addressing methods to deal with at-risk students, and effectively managing instructional technology. Of particular note to Griffin (1995) was the lack of influence of teacher involvement in school-wide decision making on individual instructional practice. Griffin attributed this curious outcome to teachers' beliefs about their own competence and the absence of reflection on their own instructional practice. The degree to which participants perceived teaching as a private enterprise and therefore not the concern of colleagues, also contributed to this finding. The accepted belief that one's teaching practice was as good as another played a role in the absence of analysis of instructional methodology. Finally, a pervasive sense from participants of having too much to do and 
not enough time to address the joint responsibilities of teaching and leading posed a barrier to tackling any additional issues.

Griffin (1995) acknowledged that many researchers would contest the value of this study primarily because of its qualitative nature and lack of large numbers of participants. Anticipating dismissal of study results, he defended the need for "a variety of inquiry modes [which] can enrich our understanding in ways that single-minded use of one research method may not" (p. 33). However, the qualitative nature of the work did not pose the most significant limitation to the study. Rather, the researcher's failure to gather data from multiple sources, i.e., interviews, observations, and official documents, limited the reliability of the findings (Bogdan \& Biklen, 2003).

While each of the previous studies focused on teachers' willingness to participate in school-wide decision making roles or the impact of participation on teacher and student outcomes, none represented long-term evaluation of these phenomena. Smylie, Lazarus, and Brownlee-Conyers (1996) conducted a longitudinal study of the instructional outcomes of participative decision making. The study examined relationships among variations in the implementation of participative decision making, instructional improvement, and student learning over a 5-year period. The study analyzed participative influence in terms of three intermediate organizational variables including teacher autonomy, accountability, and professional learning opportunities. The theoretical framework for the study suggested that participative decision making would activate change mechanisms that would improve instruction and ultimately student learning. Change mechanisms included control, motivation, and learning (see Figure 2). The control mechanism referred to teachers' sense of accountability for their work while the 
motivation mechanism meant individual teacher autonomy. The learning mechanism implied collegial interaction, support for instructional experimentation, and willingness to share ideas. The model suggested that the greater the participative nature of decision making, the more influence these three factors would exert on instructional improvement and student learning.

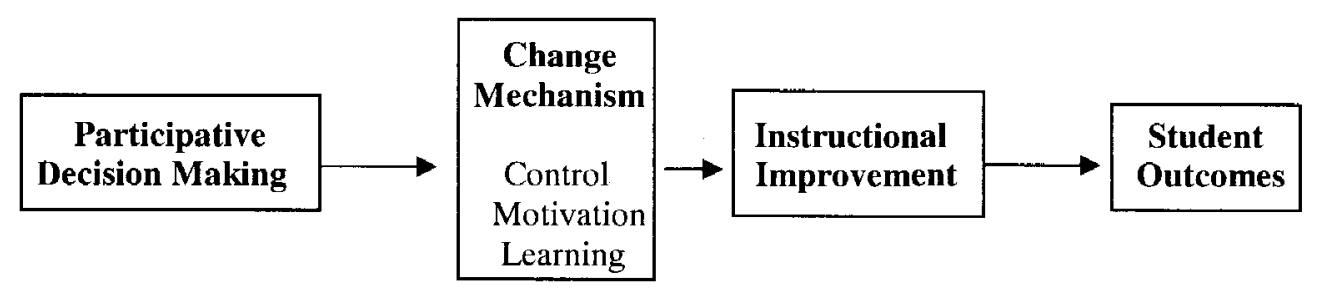

Figure 2. Theoretical framework for analyzing instructional outcomes of participative decision making.

Smylie et al. (1996) used a Midwestern metropolitan school district with seven schools averaging 3,300 students and 200 classroom teachers as the sample for the study. As a result of a collective bargaining agreement in 1989, each school had established a council. Data collection occurred over a period of 5 years, between 1990 and 1994. Faculty members in all seven schools received surveys in the spring of 1990, 1992, and 1994, corresponding to the first, third and fifth years of school council implementation. Non-responding teachers received a second survey for each of the three cycles. The 4-point Likert-type scale survey included items related to teachers' perceptions of participative decision making, individual autonomy, organizational learning, accountability, instructional improvement, and student learning. Student learning referred to student problem solving abilities, leadership capabilities, and eagerness to learn. Anchors were not identified. Response rates for 1990, 1992, and 1994 were 60\%, 79\%, and $82 \%$, respectively. Internal reliability coefficients of survey measures ranged from 0.59 to 0.94 . The researchers also conducted six to eight observations of each building 
council in the winter and spring of 1993, the fourth year of implementation. Observations provided validation of survey data as well as a deeper understanding of how teacher participation in council activities related to autonomy, accountability, organizational learning, and instructional outcomes. Observers used semi-structured protocols to focus on issues addressed by the council, teacher involvement and influence, and the principal's role on the council. Student achievement scores from the Illinois Goal Assessment Program (IGAP) standardized tests in reading and math provided data about student learning outcomes.

Data analysis occurred in three stages. Smylie et al. (1996) computed school-level means for each survey item collected during the first and last years of the study regarding measures of participative decision making, individual autonomy, accountability, and organizational learning opportunities. Computation of change scores followed in the second step. A two-tailed $t$ test determined whether the changes were statistically significant. Change scores were also determined between school-wide 1990 and 1994 IGAP math and reading scores. No tests of statistical significance were completed because these data were accessible only as school-wide averages. The third step used correlational analyses to test for relationships among the variables. The constant comparative approach provided a method of analysis of building council activity observations.

Smylie et al. (1996) found there were large, statistically significant declines in teachers' perceptions of individual autonomy across schools in the district. Results also indicated large significant increases in teachers' perceptions of accountability. Change scores uncovered small, non-significant changes in perceptions of organizational learning 
opportunities in all except two schools which displayed significant declines. While between-school results varied, teachers reported improvements in instruction and student learning as defined by the researchers. Overall IGAP reading and math achievement change scores did not show significant growth.

Smylie et al. (1996) concluded that teacher participation in school-based decision making was positively related to instructional improvement and to student learning, but had no significant impact on student achievement measured by the IGAP standardized reading and math tests. The researchers explained these discrepancies by noting that instructional improvement survey measures addressed the introduction of new programs and practices rather than improvement in existing classroom instructional strategies. Survey items which measured student learning requested subjective teacher interpretations of student problem-solving and leadership abilities rather than hard data. Thus, study results for student learning differed from results for student achievement. Generalizability was limited due to the small sample size involved in the study.

Additional studies differentiated teacher leader practices and outcomes between elementary, middle, and high schools. Stone, Horejs, and Lomas (1997) employed the case study method to examine how teacher leadership practices were similar or different among elementary, middle, and high schools. The researchers elected to integrate their case studies to compare the characteristics of teacher leadership, motivation for assuming teacher leadership roles, supports and constraints of teacher leadership, and the impact of teacher leadership on professional practice and school improvement. Selection of participants occurred by means of a survey of six elementary, six middle, and six high school teachers. Surveyed teachers indicated the names of six teachers from each school 
whom they considered teacher leaders. The case study participants were the six teacher leaders in the school identified most frequently in the teacher surveys $(N=18)$. Each of the three studies collected data using: (a) Teacher leadership Survey for Teachers at each participant's school, (b) teacher leader interviews and reflective responses, (c) informal interviews and discussions with teacher leaders, (d) informal interviews with colleagues of the teacher leaders, (e) interviews of principals, (f) teacher leaders and principals focus group discussions, (g) principals' journals, (h) document analysis, (i) archival sources, (j) observations, and (k) participant observations. Use of these multiple sources assured construct validity. The Teacher leadership Survey for Teachers consisted of multiple choice and open-ended questions. Response rates were $78 \%$ at the high school level, $76 \%$ at the middle school level, and $100 \%$ at the elementary school level. Use of patternmatching, explanation-building, and time-series analysis verified internal validity. Observations occurred over time in multiple settings by multiple observers. Participants reviewed observation and interview data for verification.

Stone et al. (1997) found that the opportunity to play a role in shared decision making was an important impetus that attracted teachers into teacher leadership. Teacher leaders at all three levels perceived themselves as facilitators and peer collaborators whose primary focus was school improvement. Primary teacher leader activities of the 18 participants mirrored this focus. Shared decision making, collaboration, and school improvement described the desired outcomes of teacher leadership activities and behaviors. All participants agreed that teacher leadership improved professional practice within their schools, and, on a personal level, increased the teacher leader's knowledge and risk-taking skills. However, no specific data indicated how teacher leadership 
improved professional practice, or to what degree this improved practice influenced student achievement at the elementary, middle, or high school level. The researchers noted lack of time, administrative support, and school climate as constraints for teacher leaders' achieving full potential.

Cameron (2005) conducted a case study to examine the tensions experienced by teachers working within collaborative decision-making structures. Collaboration referred to teachers and administrators working together to find solutions to school issues. Structure referred to the overall organization of the school which impacted how it worked and how its members interacted. Structure included the values and beliefs held collectively by school personnel. Cameron believed that the study was important because school structures could constrain or enhance instructional practice and student learning. Identifying points of tension that inhibited effective collaboration would provide insight into the collaborative process.

In order to focus more clearly on issues effecting successful teacher collaboration in a specific school-based decision making setting, Cameron (2005) conducted a theoryseeking case study, one which described the complexity of a setting without evaluating the situation in order to trigger change. The goal of theory-seeking case study is to gain insight into a specific situation. The setting for this study was an urban secondary school located on the west coast of the United States. The school consisted of an ethnically diverse student population of 800 students, 38 teachers, and 24 classified staff members. This school relied on collaborative models of interaction for decision making about teaching and learning practices within the school. The primary decision-making process was consensus building. The governing body of the school consisted of eight people: a 
volunteer parent, a student elected from the student body, five teachers elected by the teachers, and the principal. The school governing body controlled hiring, teacher development, the yearly calendar, and which decisions to send to the staff for consensus.

Cameron (2005) conducted unstructured interviews with 20 of the 38 teachers, the principal, the assistant principal, the counselor, and ten students. The interviews followed participants' streams of thought, with responses directing subsequent questions. The researcher knew most of the staff and students from previous employment at the school as a teacher, a potential source of bias. The author failed to discuss methods used to establish reliability.

Overall findings indicated four primary areas of tension for teachers. The first area of tension identified was the time required of teachers to run the school's collaborative programs. Teachers felt pulled in different directions and complained of not having enough time to work on class preparation. Collaborative work proved draining and time-intensive for teachers. The second area of tension was between the autonomy of classroom practice and the requirements of collective agreements. Teacher autonomy conflicted with the ability of departments to make collective decisions which influenced classroom instructional practice. The third area of tension existed between positional power and the norms of equal status among teachers. Teacher leaders experienced criticism about decisions from their teaching colleagues. Members of the governing body felt trapped between the positional power of the group and equal status with teaching colleagues. The principal shared school management with the governing body, but leaned toward hidden power initiatives when dealing with issues of personal and professional concern. The fourth area of tension lay with the public exposure of teacher leaders. Over 
a period of several years, the school appeared unable to generate sufficient interest among staff members to participate in the school governing body. Teachers expressed reluctance to expose themselves to conflict with teaching colleagues. Conflict occurred in the school when the governing body chose not to allow staff consensus on specific decisions, but maintained the prerogative of the governing body to decide. The intended collegial nature of the school opened these decisions to staff evaluation. Such conflicts became difficult to resolve while simultaneously maintaining a collaborative decision-making model within the school. Cameron (2005) concluded that collaborative structures have the ability to develop teaching practice, group support, and student learning experiences in a thriving environment. He cautioned, however, that tensions identified in the study must be viewed in balance with the potential of collaboration.

The studies reviewed in this section used qualitative (Cameron, 2005; Griffin, 1995; White, 1992), quantitative (Smylie, 1992a; Taylor \& Bogotch, 1994) and mixed methods (Smylie et al.; 1996; Stone et al., 1997) to reach common conclusions about teacher leader involvement in school-level decision making. Overall, the studies revealed that difficulty in dealing with colleague opposition (Cameron; Smylie, 1992a; Stone et al.) and the principal-teacher relationship (Smylie, 1992a) played significant roles in teacher willingness to participate in school-level decision making. Smylie (1992a) called for more comprehensive studies about the relationships that exist between factors that influence teachers' willingness to participate in school decision making. The strong cultural norm of individual teacher autonomy repeatedly surfaced as the primary reason for teacher reluctance to challenge peers in decision making for school change (Cameron; Griffin; Smylie, 1992a). Teachers who did choose to participate at the school level 
experienced more positive feelings about their work environment, experienced improved self-esteem (White), and became more interested in developing new and improved instructional strategies (Stone et al.; White). However, this interest did not produce significant change in classroom teachers' instructional practice (Griffin; Taylor \& Bogotch) or student academic achievement (Smylie et al.; Stone et al.; Taylor \& Bogotch). The assumption that greater student learning would naturally occur as a result of greater teacher participation in school decision making (Taylor \& Bogotch; White) was not validated. For this outcome to be achieved, the focus of decision-making groups must be instruction and student learning (Taylor \& Bogotch). Few specific, objective, measurable student achievement outcomes were designated in these studies, nor were actions of school decision-making groups linked to student achievement. None of the studies in this section yielded results which verified teacher decision making as a significant means of improving teaching practice and student learning.

\section{Teacher Leaders as Classroom Teachers}

School reformers hoped that teachers' work with school-wide decision making would translate into improved teaching and learning (Smylie et al., 1996). According to Leithwood and Duke (1999), teacher leadership parallels instructional leadership. Instructional leadership addresses behaviors of teachers when they perform activities which directly affect the growth of students. Some teacher leaders serve as full-time building curriculum leaders, coaches, or quasi-administrators. Others maintain full-time jobs as classroom teachers while assuming additional leadership responsibilities (YorkBarr \& Duke, 2004). This section addresses the strand of teacher leadership research focused on teacher leaders whose primary role is that of classroom teacher. 
Cheng (1994) investigated the relationship of the teacher leadership style on use of power, student affective performance, social climate, and perception of physical environment in a sample of primary schools. The researcher also profiled four different teacher leadership styles to deepen understanding of leadership styles and to facilitate improvement of classroom processes. Cheng used an organizational perspective to study the impact of teacher leadership on classroom practice. Defined as a social organization, a classroom included a group of students and their teacher, who acted as the leader and who wanted students to follow. Because leadership theories developed from studies of adults in organizations, Cheng wanted to know if the same theories applied to children in classrooms as they interacted with their teachers. The study used a cross-sectional survey with data taken from an ongoing large-scale research project, Education Quality in Hong Kong Primary Schools: Indicators and Organizational Determinants, conducted by the author under the auspices of a government grant. The independent variables were teacher leadership characteristics. The dependent variables included teacher use of power, student affective performance, social climate, and perception of physical environment. A pilot study involving 58 classes of 1,842 sixth grade students in 12 primary schools in the same Hong Kong school district tested instrument reliability. Sixth grade students from 678 classes in 190 primary schools in Hong Kong acted as the sample for the study with a participation rate of $100 \%$. Two groups of students randomly selected from each sixth grade class completed the surveys.

The survey instrument used in the study to measure teacher consideration behavior and initiating structure was the Leader Behavior Description Questionnaire (LBDQ). Initiating structure referred to the establishment of well-defined patterns of 
organization, channels of communication, and procedural methods. Consideration behaviors referred to the existence of friendship, mutual trust, respect, warmth, and interest in the relationship between the teacher and the class. One instrument developed in previous research measured use of power. An adaptation of another instrument assessed classroom social climate. Cheng (1994) developed the instrument used to measure students' perceptions of the quality of the classroom's physical environment, as well as the instrument used to assess affective measures of students' educational outcomes. The mediating outcomes included self-concept, attitude to peers, attitude to school, attitude to teachers, self-efficacy of learning, homework overload and drop-out intentions. Survey instruments demonstrated acceptable reliability due to use in previous research by the author.

Data analysis included correlational analysis to determine how teacher leadership styles and power exercised related to classroom performance measures. Two-way multivariate analysis (MANOVA) scrutinized initiating structure and consideration components of leadership styles on the use of power, social climate, perceived physical environment, and student affective performance. Cheng (1994) found that teacher classroom leadership and use of power strongly related to climate measures of teacher support, rule clarity, task orientation, involvement and innovation, with cumulative Eigenvalues explaining $90.9 \%$ of the variance and high correlation coefficients $(0.83)$. Classroom teacher leaders tended to use expert power $(0.48)$ and personal power $(0.55)$ rather than coercive power $(-0.40)$ to influence students. Teacher leadership style related significantly to student affective outcomes, with Eigenvalues explaining $72.2 \%$ of the variance and a high correlation (0.73). Two-way MANOVA main effects $(F=7.57$, 
$p<0.001)$ and interaction effects $(F=2.26, p<0.05)$ indicated that teacher leaders with high consideration or high initiating structure tended to influence students through expert and personal power. These results supported the belief that a significant relationship existed between teachers' observable leadership behaviors and profiles of power bases in classroom processes. The significant interaction effect on expert power $(F=4.48$, $p<0.05)$ emphasized that high consideration was more important than high initiating structure. However, the contribution of initiating structure in a low consideration group was stronger than in a high consideration group. Two-way MANOVA analysis of the effects of leadership styles on classroom environment and student affective performance yielded significant main effects $(p<0.01)$. Students taught by a high consideration teacher tended to be more satisfied with the classroom's physical environment and to possess positive perceptions about the class. The main effects of consideration on measures of student affective performance, specifically in attitude to school, attitude to teachers, self-efficacy to learning, feeling of homework overload, and intention to drop out, were significant $(p<0.001)$.

Averaging the standardized scores of all dependent variables within each leadership style group provided Cheng (1994) with data for a leadership style profile map. Map analysis indicated that classroom teacher leaders emphasized both task achievement and relationships with students and that teacher leaders relied upon expert and personal power. Students' perceptions of these teachers' classrooms were that they were comfortable, clean, and conducive learning environments. The social environments in these teachers' classrooms were positive, friendly, supportive, and orderly, with organized activities and assignments, a clear set of rules, innovative teaching strategies, 
and emphasis placed on accomplishing the learning tasks. Students in these teachers' classrooms felt confident and competent and indicated that it was unlikely that they would drop out of school. The significant relationship between leadership style and classroom performance in climate and affective measures supported the need for a balanced leadership style. Both task orientation and relationship-building behaviors were effective in achieving desired outcomes. A leadership style of high consideration and high initiating structure was the preferred method for leading students. These findings suggested that generalization of ideas and theories developed from adult organizations could be applied within the context of school classrooms.

Transferring task-orientation and relationship-building behaviors from adult organizational context to a student classroom context proved to be appropriate. For teachers adept at working in a student classroom context, the transfer of task-orientation and relationship-building behaviors into a collegial setting posed challenges. Ovando (1996) studied teacher leaders' perceptions associated with their twofold duties of teaching and leading to determine the opportunities and challenges posed by working in a dual capacity. Teacher leader meant a teacher with leadership duties in a school-based management environment in addition to classroom teaching responsibilities. Teaching practice meant normal teaching duties such as planning and implementing instruction in a classroom setting. A school district in Texas known for successful 3-year implementation of a Peer Assisted Leadership and Support Program and school-based management structure supplied the sample for the study. Flexible criterion-based sampling yielded 25 teacher leader participants from a pool of 132 teacher leaders. The sample represented different levels of teaching and diverse teacher leadership positions. Data collection 
included administration of an open-ended questionnaire to gather teacher leaders' insights and perspectives. The researcher chose the open-ended questionnaire method to eliminate pre-categorization of responses. The instrument included questions related to leadership responsibilities, time of leadership performance, preparation for leadership development, influence of leadership duties on teaching practice, and satisfaction with working in two roles. Data analysis included synthesis of responses followed by content analysis for pattern identification.

Ovando (1996) found that teacher leaders had a variety of leadership opportunities. The range of positions reflected the unique characteristics of each school. Lack of time to carry out leadership tasks so that the quality of classroom instruction did not suffer posed a significant challenge. Teacher leaders in the study maximized opportunities for professional growth, but needed on-going leadership skills development with continuous feedback. Teacher leaders indicated mixed reactions about combining leadership and teaching responsibilities. While leadership opportunities generated insight and new teaching ideas, time spent engaged in leadership duties detracted from a clear focus on classroom instruction. All participants experienced a reduction in school time for planning and preparing for instruction, as well as increased use of personal time to accomplish school tasks. Teacher leaders indicated satisfaction and enthusiasm to some extent regarding dual roles. While teacher leadership provided teachers with opportunities to influence school-wide leadership in site-based decision-making schools, teacher leadership created challenges related to teacher work load, pressure, responsibilities, and increased work time. Ovando (1996) recommended that site-based managed schools reduce the challenges and improve the opportunities for teacher leaders 
to better serve students' needs. Ovando also called for further in-depth studies of teacher leadership effects to identify the benefits of teacher leadership for improving teaching and learning.

Similar to Ovando (1996), Silva, Gimbert, and Nolan (2000) conducted three exploratory case studies to understand how teachers experience leadership from inside the classroom. The researchers' definition of teacher leadership stemmed from what was termed the third wave of teacher leadership literature. The first wave focused on formal teacher leadership roles, (i.e., department head, head teacher, or master teacher), all utilizing teacher leaders in managerial roles. The second wave dealt with teachers as instructional leaders in team leader, curriculum developer, or staff development teacher positions. Both of these trends took teachers out of classrooms and removed them from direct interaction with students. The third wave definition addressed teacher leadership as part of a teacher's daily work with children, but also included collaboration with other teachers to explore, investigate, discuss, and share any practice that would support teaching and learning. The researchers described member choice as a unique case selection procedure, which based participant selection on a specified characteristic intrinsic to the population. Each participant met four criteria based upon the unique case selection procedure: (a) a minimum of 10 years' teaching experience, (b) considered and named a teacher leader by at least three colleagues within the school district, (c) primary responsibility viewed as being a classroom teacher, and (d) a history of serving the district in an acknowledged leadership task. Teachers in the district nominated possible participants who met the established criteria through phone interviews conducted by the researchers. Telephone interview results led to the acceptance of all three nominated 
teachers for participation in the study.

Silva et al. (2000) collected data using 90- to 120 -minute semi-structured interviews with each subject. Additional probing questions allowed for clarification and elaboration. Interviews focused on background biographical information, the teacher's experiences as a teacher leader, and observations about teacher leader prospects in the school district. Tape-recording and transcription of interviews followed. Each participant received a copy of transcribed interview data. Data analysis included repeated readings of interview transcriptions to uncover over-arching themes within and between each case. An unbiased research colleague interviewed each participant and reviewed all transcripts to afford trustworthiness of data. The work of the unbiased interviewer/reviewer eliminated the need to address inter-rater reliability. Within-case analysis indicated that (a) principals sometimes became barriers to teacher leadership, (b) school structures and schedules appeared to be more valuable than people, and (c) there was insufficient time and opportunity for teachers to share knowledge and plan together. Between-case analysis yielded five assertions about teacher leadership: (a) teacher leaders navigate school culture and organizational structures in various ways; (b) teacher leaders nurture relationships; (c) teacher leaders encourage professional growth; (d) teacher leaders help others with change; and (e) teacher leaders challenge the status quo on behalf of the needs of their students.

Based on these findings, Silva et al. (2000) recommended that professional development schools provide training in navigating school organizational structures to teacher interns and mentors. The researchers further suggested that principals needed to learn how to allow teachers to exercise collaborative decision making for impact on their 
students' needs. Development of teachers must become more valuable than efficient school structures.

The assertions of Silva et al.(2000) about teacher leadership reinforced the benefits of teacher leader interactions with colleagues and students. Sawyer (2001) conducted a longitudinal study of three teachers to examine how each teacher deepened a sense of purpose and skill by developing collaborative skills. The researcher noted that collaboration can create a climate of mutual security where professional openness can flourish so that instructional practice might improve and result in higher levels of student learning. Data for the study came from a larger 10-year longitudinal study about recruitment, preparation, teaching, induction, and retention of alternate route and college prepared teachers. Participants included one middle school mathematics teacher, one high school mathematics teacher, and one high school English teacher. All participants worked at different schools and did not know each other. Data collection consisted of gathering participants' self-reports of their work as well as results of four surveys and semistructured interviews. The participants completed four surveys and participated in four interviews during the first 6 years of the study. Over the next 4 years, respondents participated in four more interviews.

Sawyer (2001) used both descriptive and explanatory narrative. Descriptive narrative served to describe accurately the interpretive, narrative accounts that participants used to develop sequences of events in their lives in order to make them meaningful. Explanatory narrative explained the connections between the events as cause and effect. Each participant received a reconstruction of their collaborative history over their first 10 years of teaching. Narrative data in these reconstructions included 
information from interviews and surveys. The participants interpreted and discussed their perspectives of how their collaborative lives evolved. The narrative served as a phenomena being studied as well as a method of studying the data. Data analysis occurred in a multi-step process. After editing of initial interviews and coding of the data, Sawyer indicated that he "held a conversation with the data" where he looked for patterns. The researcher then arranged organized units of data from interviews into chronological order, assigned primary codes, and identified primary themes. The researcher re-read interviews to identify additional or more meaningful examples of themes. The final collaborative history reconstructions woven into narratives provided material for discussion between the participants and the researcher for member checks. Sawyer (2001) found that each teacher shared a deep concern for the learning of all their students. Each teacher initiated collaborative work because of questions about teaching. Each teacher demonstrated a willingness to expose her work to public scrutiny and each believed in experiential learning. All participants believed that a shared philosophy of teaching and learning was more critical to collaboration than common teaching practices. Participants discovered in the first 3 years that the greatest motivation for collaboration was their awareness that cooperation improved their instructional practices. Motivation shifted to curriculum development by the fourth year. Each teacher eventually established a pattern of collaboration by which to evaluate a variety of curriculum approaches. As collaboration evolved, so did each teacher's approach to instruction. Each became more tolerant of the ambiguity within themselves and the curriculum and more knowledgeable about instructional practice. While participants reported that administrative and departmental assistance for collaboration was essential, 
each teacher acknowledged different degrees of support. The collaborative process itself provided strong motivation for continuing engagement in collaboration. All three teachers' practical knowledge of teaching improved, and all three developed a greater understanding of the support provided by existing school structures.

Sawyer's (2001) conclusions about the evolution of teachers' collaborative abilities through practice over time led to further investigation about teacher leader behaviors which impacted classroom practice. Cruz (2003) examined the characteristics and behaviors of middle school mathematics teacher leaders in their classrooms using a mixed methods design. The population consisted of 112 middle school mathematics teachers in four school districts in western Washington. Each of these 112 middle school mathematics teachers received a one-page questionnaire for the purpose of naming teachers whom they considered teacher leaders in the middle school mathematics classroom. Questionnaire response rate was $50 \%(N=56)$. The questionnaire solicited information about special characteristics found in those teachers named as leaders in the middle school mathematics classroom. Responses indicated that identified teacher leaders were clearly articulate in their teaching, approachable, personable, creative, innovative, flexible, fair, and always willing to share ideas. These teacher leaders' primary focus was student learning.

From the 20 teachers nominated through the questionnaire, 15 agreed to be interviewed. The interview gathered demographic and educational background information about each participant, as well as data about teaching experience and use of technology in instruction. Questions also inquired about math programs and types of instructional strategies used for instruction. Common methods of assessment, a personal 
definition of teacher leadership for middle school math teachers, and leadership abilities essential for middle school mathematics teachers completed the question topics included in the interview portion of the study. The Teacher Roles Observation Schedule (TROS) served as a data gathering tool for the ethnographic observations of 14 participants. This instrument helped describe the nature and patterns of teacher behaviors within instructional programs. The researcher used the TROS to record teacher leaders' classroom behaviors in non-continuous, 60 -second interval observations. Recorded observations were 4 minutes apart.

Cruz (2003) found a pattern of questioning, explaining, commenting, and listening among the mathematics teacher leader instructional behaviors when interacting with students and named it the Quadad Cycle. The Quadad Cycle referred to a union or grouping of four closely related teacher behaviors associated with the nature of teacherstudent interaction. After member checks, Cruz concluded that this process described active communication. Additional findings of the study indicated that teacher leadership could not be summarized in a one-sentence definition. Teacher leadership encompassed mentoring, facilitating, sharing ideas, materials, and teaching strategies to support the learning of the greater school community. Teacher leadership included behaving in positive, caring, and encouraging ways because of a love of students and content. Cruz (2003) determined that teacher leaders spent more time teaching the whole class $(67 \%)$ more so than small groups $(13 \%)$ or individual students $(20 \%)$. Observation patterns showed that teacher leaders attended to, in descending order, task products $62 \%$ of the time, the task process $56 \%$ of the time, task procedures $21 \%$ of the time, and task content $11 \%$ of the time. Interaction with students in an instructional format occurred 
$93 \%$ of the time. Use of a variety of instructional strategies was evident, such as direct instruction, cooperative learning, and real-world problem-solving. Methods of assessment varied as well, including observations, classroom tests, portfolios, interviews, and projects. Use of technology for instruction was common but did not include computers due to lack of time with math curriculum and lack of appropriate software. All observed teacher leaders provided students with the help and materials needed for success and exhibited respectful behaviors toward each student. Teacher leaders noted that their goal was to introduce math content and skill to students where students could learn it. In this study, Cruz did not investigate the differences between teacher leader classroom behaviors and practices and those of regular teachers. A comparison of this nature would be of interest.

In another study of classroom teachers who acted as teacher leaders, Middlebrooks (2004) examined teacher professionalism and leadership in a mixed method study. Acting as a non-participant observer, the researcher conducted interviews and observations focusing on leadership and professional behaviors that described teachers as teacher leaders in secondary schools. Descriptive statistics reported response patterns to interview questions. The sample consisted of 43 comprehensive secondary schools, 35 in the New York boroughs of Brooklyn, the Bronx, Manhattan, and Queens, and eight schools in the metropolitan area of Atlanta, Georgia. All schools in the sample followed the traditional school organizational model with one principal who managed and supervised all programs and personnel.

Middlebrooks (2004) conducted interviews with a total of 185 students in focus groups ranging in size from 3 to 16 . Administrators or teachers selected the students for 
the focus groups. Fifty-two administrators participated in individual interviews before the classroom observations. Ninety teachers participated in interviews after classroom observations. School personnel at each school chose the teachers for the observations. Each classroom observation was approximately 50 minutes long. Data analysis consisted of organizing and coding information to identify emergent themes related to teacher leadership and professionalism, with attention focused on the unique perceptions of students, teachers, and administrators. Identification of sub-themes helped organize and interpret the data. The researcher's use of a narrative reporting style knitted relevant literature together with findings from the study.

From his study, Middlebrooks (2004) confirmed the importance of leadership skills and professional behavior. Teachers in the study demonstrated leadership in the academic setting and acted in the roles of manager, decision maker, role model, change agent, and advocate. The opportunities afforded teachers to use leadership skills affirmed the need for leadership preparation in teacher preparation programs. Interview results also showed that students, teachers, and administrators agreed that teaching students leadership skills ensured their success in any career. Leadership skills included communication, thinking, interpersonal, and social proficiencies.

Teacher leadership skills and professional behavior in the classroom setting continued to intrigue researchers. Katyal and Evers (2005) assumed a unique approach toward teacher leadership in their qualitative study of three schools in Hong Kong. These researchers classified teacher leadership as the holistic influence that teachers had on students, regardless of the teacher's formal or informal leadership role within the school. Participants consisted of 14 teachers, 12 parents and 3 student groups of approximately 
10 students per group.

Although Katyal and Evers (2005) reported no information regarding time frame for the study or number of contacts with participants, interviews revealed that leadership was not principally about the teacher's role with other teachers, but about the relationships and connections with the students in the school. Participant data indicated that effective teachers were committed to their students, fair, empathetic, supportive, approachable, hardworking, and possessed good communication and instructional skills.

In a movement away from definitions of teacher leaders as efficient managers, instructional guides, or collegial professionals, Katyal and Evers (2005) concluded that teachers are leaders who do not need the sanction of empowerment from outside sources, i.e., administrators or peers. Teacher leadership constituted actions between teachers and their students through instruction and social modeling, rather than between teachers and their colleagues. Effective teachers made learning in school authentic and meaningful through connection to real-life application. Socialization comprised an equally important part of the teacher leader definition. Through their personal values and beliefs, as well as their work ethic, teachers exerted significant influence on their students. Teacher leadership signified the continuous process of shaping student engagement regardless of organizational titles or structures.

Six of the seven studies in this section were primarily qualitative in nature, describing the characteristics, concerns, and general experiences of classroom teachers who also act as teacher leaders. However, the researchers reviewed in this section did not define the focus of teacher leadership in the same way. Cheng (1994) and Katyal and Evers (2005) viewed teacher influence on students as the focal point of all teacher 
leadership. Classroom management style related significantly to student affective outcomes (Cheng), thus teachers who were successful classroom leaders relied upon expert and personal power rather than positional power to create an effective classroom environment. Teacher leadership was not about the impact of teachers on other teachers, but the holistic influence that teachers had on students through instruction and social modeling (Katyal \& Evers). Cheng's study, however, did not make a connection between teachers leading students and teachers leading teachers. One might hypothesize that the same leadership characteristics of high consideration and high initiating structure which proved effective with students could also be successful in leading collaborative experiences with other teachers. However, there was neither the intent nor the evidence to make that leap in this study.

The remaining five researchers reviewed in this section (Ovando, 1996; Silva et al., 1996; Sawyer, 2001; Cruz, 2003; Middlebrooks, 2004) approached classroom teachers as teacher leaders from the perspective of how these teachers influenced other teachers while simultaneously fulfilling the obligations of a full-time classroom teacher. This approach yielded common conclusions. Teacher leaders who combined teaching responsibilities with additional leadership responsibilities experienced mixed reactions (Ovando; Silva et al.). Opportunities to collaborate with other teachers (Silva et al.; Sawyer; Cruz), to develop knowledge and insight about instruction (Sawyer) and to influence school-wide leadership decisions were offset by additional work load, less personal time, and loss of undivided attention to classroom instruction. However, classroom teacher leaders saw their work as important for the growth of their students and their school (Cruz; Katyal \& Evers; Middlebrooks; Ovando; Silva et al.). While these 
studies together described the characteristics of teacher leader performance from the perspective of two different definitions, the qualitative nature of the work could not yield measurable results of the impact of the classroom teacher leader on student learning.

\section{Teacher Leaders as Instructional Change Agents}

Katzenmeyer and Moller (2001, p. 17) refer to teacher leaders as those who "influence others toward improved educational practice." Spillane et al. (2003) describe instructional leadership as the influence exerted over teachers' instructional practices. Lambert (1998) explains instructional leadership as building capacity by "learning together, constructing meaning and knowledge collectively and collaboratively...so that a school community can keep moving when current leaders leave" (pp. 5, 8). York-Barr and Duke (2004) assert that educational improvement at the instructional level must involve leadership by teachers. The hope is that teacher leaders will move schools toward continuous improvement in teaching and learning (York-Barr \& Duke). The studies in this section investigate the impact of teacher leaders on the instructional practices of their colleagues.

In a study about the perceptions of teacher leader functions, Smylie and Denny (1990) examined the definition and performance of teacher leadership roles, including peer reactions which could impact both role definition and performance. The sample included 13 teacher leaders from a K-8 metropolitan school district. Five of the teacher leaders worked at the primary level, five worked at the intermediate level, and three worked at the middle school level.

Smylie and Denny (1990) utilized a systematic, multi-stage, interactive method of data collection, analysis, and interpretation. In the first stage, each of the teacher leaders 
participated in unstructured, tape-recorded interviews. Questions focused on how each teacher leader characterized the role, the specific leadership activities each initiated and guided, and issues each felt impacted the evolution and performance of their role as teacher leaders. Recurring themes emerged from these data through comparative analysis. The researchers then validated these themes by submitting them for evaluation to four independent university educational leadership researchers and district-level personnel responsible for developing the teacher enhancement initiative. Codification of themes into written Likert-type questionnaire items followed. The first section of the questionnaire addressed teacher leaders' perceptions of their specific leadership roles with anchors ranging from $1=$ not at all to $5=$ to a very great extent. The second questionnaire section measured activities of teacher leaders by time spent, with anchors ranging from $1=$ least time spent to $5=$ most time spent . The third section evaluated certainties and uncertainties associated with teacher leadership roles, with anchors ranging from $1=$ very uncertain to $4=$ very certain . The fourth questionnaire segment reviewed teacher leaders' needs related to performance of their leadership roles, with anchors from $1=$ not at all to $5=$ to a very great extent . The final questionnaire section gathered information about tensions that teacher leaders experienced in performance of leadership duties with anchors ranging from $1=$ not at all to $5=$ to a very great extent . The 13 teacher leaders responded to the survey allowing researchers to compare these results to previous interview results with this same group for verification and validation. The researchers then discussed the results of interviews and surveys with the 13 teacher leader participants.

The second level of data collection consisted of the administration of surveys 
regarding classroom teachers' perceptions and reactions to teacher leaders. Ninety randomly selected teachers from the school district participated. The survey consisted of open-ended questions asking participants to recount the roles that teacher leaders played in their schools; activities in which teachers had participated with teacher leaders; and any benefits to the school, the district, or themselves as teachers that resulted from interactions with teacher leaders. The 90 participating teachers received the surveys through the mail. A total of 56 teachers returned usable surveys, for a response rate of $62 \%$. Recurring themes again emerged from the data through comparison analysis. Smylie and Denny (1990) employed the appraisal skills of the same four educational leadership university researchers and the same district level personnel to validate and verify identified themes. The researchers shared survey theme outcomes with representatives of survey participants for validation.

From this study, Smylie and Denny (1990) found that teacher leaders viewed their primary role as helping and supporting the work of teachers in their buildings through bolstering teachers' daily work with students and facilitating improvement in instructional practice. Ironically, further findings revealed that teacher leaders in the study spent the least time working in these capacities when compared to time spent on other job responsibilities. The researchers contended that lack of adequate time during the school day prevented access for interaction between teacher leaders and teachers in the school. Teacher leaders also appeared hesitant to challenge the norms of privacy and autonomy among teachers. Teacher survey data indicated a significant disparity in perceptions about what teacher leaders do and should do. Contextual organizational factors may strongly influence the definition and performance of teacher leaders; 
therefore, teacher leadership development should be approached as an element of organizational change and not only as a way to improve one teacher's leadership capacity. The researchers noted that teachers themselves may be one of the greatest barriers to the success of teacher leaders.

In a separate study, Smylie (1992b) investigated organizational and psychological factors that influence interactions between teachers and teacher leaders regarding classroom instruction. One hundred and sixteen out of a possible 200 teachers from a midwestern metropolitan school district participated in the survey study. The interval level dependent variable was the interaction between teachers and teacher leaders. The interval level independent variables included opportunities for interaction between teachers and the teacher leader in the school, the school's social context, and teachers' beliefs concerning teachers' working relationships. Opportunities for interaction referred to how accessible building teacher leaders were to teachers. School social context referred to what the school culture dictated about teacher/teacher leader interactions. Beliefs derived from six variables found in previous studies related to teachers' professional interactions: (a) norm of professional equality, (b) norm of professional autonomy, (c) norm of privacy, (d) opposition to peer judgment, (e) belief that giving and receiving advice imply status differences, and (f) belief that receiving advice implies obligation. Teachers responded to these variables on a 4-point Likert-type scale survey. A factor analysis revealed significant inter-correlation among the six variables related to teacher beliefs.

Smylie (1992b) asked teacher participants to mark all items on a 20-item checklist which best described their interactions with building teacher leaders during the past year. 
The types of interactions included (a) observation and discussion of instructional methods, (b) developing curriculum or instructional tools, (c) learning and practicing new instructional strategies, (d) developing classroom management techniques, and (e) brainstorming solutions for meeting the needs of individual students. Then, from the list of items checked, teachers selected and ranked the five interactions from the survey in which they most frequently engaged with teacher leaders. Rankings of these top five interactions were added to determine relative frequency of occurrence. A square root transformation normalized the distribution of cases before further analysis.

Least squares multiple regression procedures disclosed three statistically significant relationships $(p<.01)$ between teachers and teacher leader interaction and variables representing opportunities for interaction, school social context, and psychological orientation. Advice implying obligation demonstrated the greatest influence on teacher interaction with teacher leaders $(\beta=-.3328)$. Professional equality $(\beta=-.2363)$ and opportunity for interaction $(\beta=-.2240)$ emerged as significant influences as well. The more strongly teachers believed that receiving advice from a teacher leader implied obligation to implement recommendations and the more strongly teachers believed in professional equality among teachers, the less likely interaction regarding classroom instruction would occur between teachers and teacher leaders. An increase in the number of opportunities to interact resulted in a greater number of reported interactions. Smylie (1992b) concluded that teachers' beliefs about interdependence and professional status proved to be primary predictors of the level of interaction between teachers and teacher leaders. Professional working relationships already in existence in schools where teacher leadership roles are then introduced do not 
necessarily predict the development of positive, collaborative relationships between teachers and the teacher leader.

Interactions between teachers and teacher leaders pose new dilemmas when teacher leadership roles deviate from historically-accepted positions. Little (1995) conducted a 2-year case study of the development of teacher leadership in two high schools heavily involved in restructuring. Participant selection relied upon high school restructuring grant award recipients meeting three criteria. These criteria included (a) evidence of efforts to design a rigorous core curriculum for all students, (b) efforts to develop unity and natural connections across core curriculum, and (c) processes to support a successful school-to-work transition. Data collection consisted of interviews of 53 teachers, including 21 past and present teacher leaders. Open-ended, semi-structured interview questions probed participant views and experiences of leadership. Observations of teachers engaged in committee meetings, planning sessions, in-service activities, as well as informal observations of a regular work day, supported interview data. Analysis of demographic data, restructuring records, and faculty teaching responsibilities contributed to data triangulation.

Little (1995) determined that each high school approached newly designed, crosssubject leadership roles differently; thus, new interpretations of the responsibilities of department heads resulted. In the first high school, principal-appointed teacher leaders led cross-subject departments and joined teachers in interdisciplinary partnerships in two broad divisions. These cross-subject partners received support through scheduling of classes and common planning. Partnered groups of interdisciplinary teachers met regularly to discuss curriculum and assessment. The broadened school leadership 
structure consisted of two division teacher leaders and a number of associate teacher leaders responsible for individual subjects (see Figure 3). Associate teacher leaders were former department heads. Content expertise did not determine teacher leadership selection.
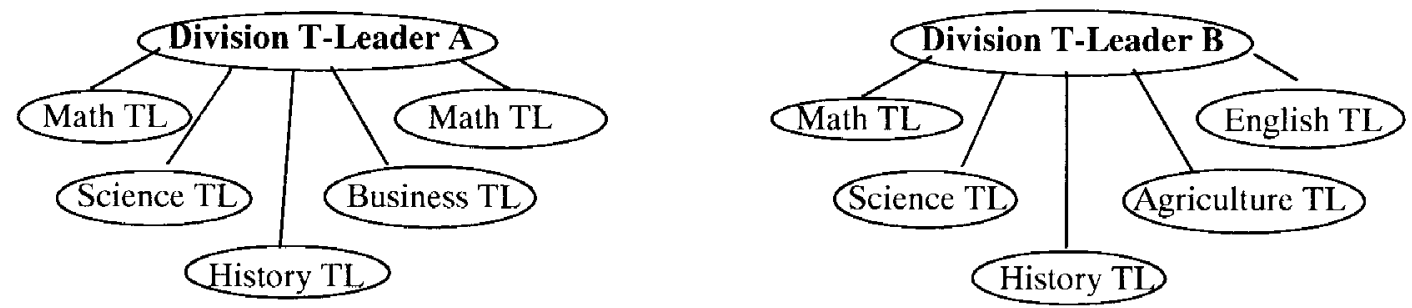

Figure 3. Framework for interdisciplinary cross-subject high school leadership partners.

In the second high school, designated team leaders appointed by the principal led interdisciplinary teams. These team leader positions supplemented other individuals retained in department chair positions, which Little (1995) referred to as the most common form of teacher leadership. Department heads cited content expertise and teaching experience as criteria for leadership. Little found that this non-conventional approach resulted in tension between team leaders and department heads. Although perceived as ineffective by content teachers and department heads due to lack of content expertise, team leaders did possess knowledge regarding restructuring procedures. Team leaders met each week with the principal, assistant principals, and counselors.

Department heads met periodically with the principal.

Little (1995) concluded that while the two approaches to school restructuring were compatible, the latter approach challenged the historical culture and the autonomy of department heads as content specialists. Teaching within and across contents could provide a more balanced learning experience for high school students. However, tension 
developed between department heads and team leaders. Separating leadership roles from traditional content-specific departments and assigning leadership authority to the development of common instructional practice among disciplines presented significant challenges to both groups.

Peer acceptance of a colleague's leadership for instructional change describes challenges faced by teacher leaders who act as peer coaches. Kohler and Crilley (1997) examined the effects of peer coaching on teachers' adoption of a new instructional practice and the processes connected with adopting new practices. The independent variable was peer coaching; the dependent variable was change in classroom instruction. For the purpose of this study, peer coaching referred to observation of a prescribed lesson format, followed by reflective discussion of the lesson's success, and culminating with identification of how to improve the successful implementation of lesson procedures in order to improve student learning. The participants consisted of four elementary teachers selected by the director of curriculum and instruction. A retired teacher familiar with peer coaching strategies served as the peer coach for the study. The four teachers received training about the goals and activities of the study and then selected the curriculum to be taught. Required lesson structure, based on a direct instruction model, included a minilesson, reciprocal learning, and closure activity.

Kohler and Crilley (1997) collected data by gathering information around four themes. The first theme consisted of an 11-item checklist which assessed how teachers organized and conducted the lesson. The second theme encompassed instructional processes utilized in delivery of the lesson. A predetermined coding system accounted for frequency of instructional functions, teacher behaviors, student behaviors, subject matter, 
and student groupings. Observations centered on one student each day based on random selection. The third theme involved use of a checklist to appraise how teachers cooperated with the peer coach with regard to lesson organization, student performance, and other content variables. The fourth theme dealt with a seven-statement survey which addressed the participants' level of satisfaction with the lesson approach. The survey utilized a 5-point Likert scale with anchors ranging from 5 agree strongly to $1=$ disagree strongly. Participants responded to the survey after every four to five lessons. A multiple-baseline research design allowed for sequential and staggered administration of the survey conditions across teachers. After the initial participant training, each teacher completed the baseline phase. This phase consisted of each teacher independently planning how to implement the required lesson structure. Teachers participated in assessments regarding the organization of the lessons and observations of lesson procedures during this phase.

One teacher continued to the peer coaching phase, while the remaining three maintained the baseline phase. The peer coaching phase included seven collaborative sessions between the teacher and the peer coach. The peer coach monitored and facilitated student participation during the lesson, then met with the teacher following the lesson. The peer coach and teacher discussed issues regarding the lesson and brainstormed ideas for improvement. Each teacher successively entered the peer coaching phase. The final phase involved maintenance, which matched the independent work level to the baseline condition. Teachers worked without assistance from the peer coach. Kohler and Crilley (1997) determined that teachers made more procedural changes in the peer coaching phase than in the initial independent work phase. Areas that teachers did 
not discuss with the peer coach remained unchanged. The researchers concluded that (a) teaching processes could be reviewed each day, (b) instructional change was more likely to occur with collaboration rather than working independently, and (c) instructional change could be maintained over time. Kohler and Crilley found that the processes occurring during the lesson procedures were fitting for the lesson purposes. Reciprocal learning, characterized by student talk with a partner, proved successful in generating student engagement and learning. Peer coaching resulted in a positive impact on teacher practice and subsequently on student engagement. However, the researchers did not include data to validate how student learning improved.

Kohler and Crilley (1997) found that peer coaching facilitated instructional change. School conditions that promote openness between teaching colleagues are a necessary precursor to peer coaching collaboration. Beattie (2002) conducted a qualitative research study to describe how teacher leaders create a culture of connectedness and creativity to allow the development of shared values and a common vision that coincide with the growth of individual teacher independence, interdependence, and student commitment. The participants consisted of teacher leaders at Corktown Community High School, 1 of 10 alternative secondary schools in a large urban Canadian school district, and the site of previous investigation by the researcher. Because one principal administers the alternative schools, all teachers at Corktown Community High School take 2-year turns serving as teacher leaders and share administrative responsibilities normally handled by the building principal and assistant principal. Teacher leaders at Corktown have a lighter teaching load in order to address their additional administrative tasks. Data collection included two-hour, open-ended interviews 
with each current teacher leader. Classroom and administrative observations of these same individuals from the researcher's previous study at Corktown Community High School provided additional information. The constant comparative method of data analysis revealed recurring themes.

Beattie (2002) determined that teacher leaders placed high priority on modeling behaviors, skills, and attitudes that lead to developing supportive relationships, ongoing dialogue, and shared decision-making among teachers and students. Teacher leaders reported physical, emotional, and intellectual tensions due to scarce teaching resources. Creating a culture of collaboration, connectedness, and commitment to the community required teacher leaders to develop their own personal competencies to respond, reciprocate, and connect with teachers and students. Authenticity and openness about their work, their dealings with others, and their own professional growth required acknowledgement and ownership of personal and professional imperfections. In short, teacher leaders gained respect and influence through transparency. The downside of this transparency was the demand of the teacher leader's time, energy, and willingness to treat everyone with respect regardless of circumstance. Exhaustion, health problems, lack of time to do needed work, and a marked imbalance between professional and personal life were typical among them. Teacher leaders remained committed to those whom they served as teacher and administrator and accepted these dilemmas as part of life.

While Beattie (2002) found that teacher leaders effected most change with colleagues through transparency of their attitudes and practice, Frost and Durrant (2002) investigated how teacher leaders could best facilitate teacher-led work, conducting a qualitative study to develop a framework of ideas to clarify factors that should be 
considered when planning teacher-led development work and assessing the impact of that work. A purposive sample of 12 teachers comprised the study participants. Each teacher was already a member of one of three programs supporting teachers in school improvement endeavors. These programs were familiar to the researchers and provided participants experienced with launching and cultivating change for growth within their schools.

Frost and Durrant (2002) collected data through interviews and mining of documents. The researchers each conducted one interview using exploratory questions. A research officer used the same interview protocol with the remaining 10 participants.

Recording, transcription, and coding of all interviews by the research officer followed. The coding process respected the configuration of a previously established, tentative framework. Each participant also provided planning documents, portfolios, and graduatelevel research that supplied evidence of completed teacher-led development work (See Figure 4).

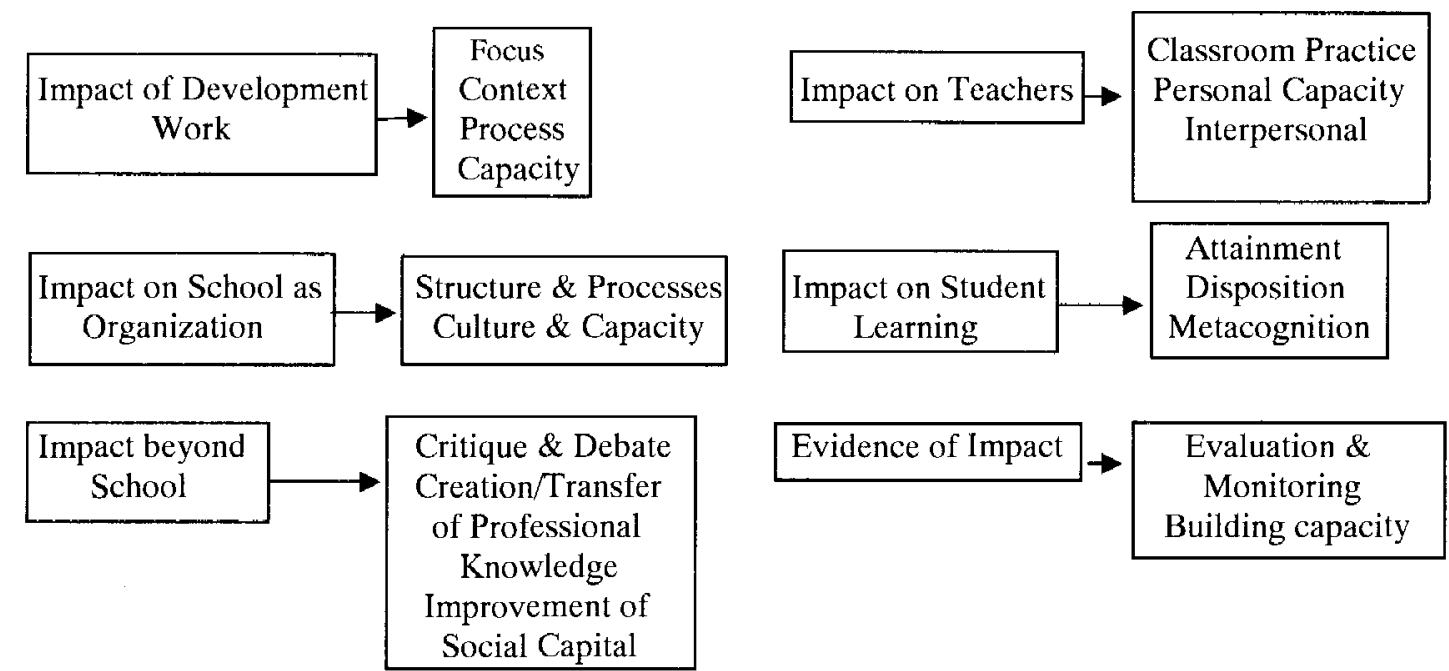

Figure 4. A conceptual framework for the impact of teacher-led development work. Data analysis of interviews and documents resulted in the development of a 
revised framework of ideas that could be used to design instruments, workshop strategies, and additional materials to support and assess teacher-led development work. The revised conceptual framework on the impact of teacher-led development work listed six main topics and several subordinate ideas for each topic. Focus, context, and process were subtopics of factors which could affect the impact of development work. Classroom practice, personal capacity, and interpersonal capacity described the impact on teachers. Structures and processes, followed by culture and capacity, described the impact on the school-as-organization category. Critique and debate, creation and transfer of professional knowledge, and improvements in social capital in the community completed the impact beyond-the-school topic. Attainment, disposition, and metacognition described the factors impacting pupils' learning. Evaluation, monitoring, and building leadership capacity described processes for measuring impact. Frost and Durrant (2002) concluded that support of teacher leaders is essential for improvement in student achievement. The framework developed as a result of their study could be used as a tool to support teacher-led development work (See Figure 4).

While Little (1995), Kohler and Crilley (1997), Beattie (2002), and Frost and Durrant (2002) each examined teacher leaders as instructional change agents with qualitative methods, Miller (2002) used a different qualitative approach, choosing to explore the role of school-insider-change-leader with naturalistic, ethnographic methods. Because Miller occupied the role of researcher and change leader within the school context, he recognized the need to eliminate bias through triangulation of methods, data, investigator, and theory. Based on the research of Denzin (1989), Miller understood that ...to limit bias in the data, multiple research methods should be used ... since any element of interaction - time, rules, relationships, objects, characteristics, 
observers and observed - can cause distortion. He further argued that besides methodological triangulation as a means of limiting bias in the data, other forms of triangulation should be considered, including data triangulation,investigator triangulation, and theory triangulation (p. 345).

Thus, Miller (2002) used questionnaires, field notes, interviews, and documents to triangulate methods. Data triangulation was accomplished by (a) soliciting interview information from specifically selected individuals and gathering questionnaire data from all staff, (b) collecting field notes, questionnaire, and interview data at different intervals over a 4-year period, and (c) gathering questionnaire data in the same locations at different times and recording field notes and interview data in a variety of locations. Investigator triangulation occurred through the collaborative observations of a colleague regarding field notes. Theory triangulation was accomplished by reviewing theories of culture and change from three different researchers.

Change referred to changes in teachers' practices and behavior, evident in attitudes and thinking. Participants in this ethnographic case study consisted of the faculty, staff, and students in an international school serving students aged 11-18 over a 4-year period. Data collection consisted of structured interviews with a balanced number of males and females who held a variety of positions in the school. All staff members completed questionnaires. Document analysis and recording of field notes completed the data collection process.

Miller (2002) concluded from the study that teachers are intrinsically and morally motivated; thus, leadership for change must emanate from credible, moral authority. Credible insiders who possess such moral authority can lead change provided they maintain a vision that builds upon and surpasses the existing culture. Sustaining interactivity between the current culture and the insider change leader is critical for 
change to become institutionalized.

Spillane, Hallett, and Diamond (2003) expanded upon the credible, moral authority identified by Miller (2002) as a prerequisite for effective teacher leadership. Spillane et al. reviewed how different types of capital affected teachers' construction of instructional leadership in specific contexts. The operational definition of instructional leadership used in this study was the influence exerted over teachers' instructional practices. The researchers posited that leadership is an interactive process where followers designate others as leaders based on valued forms of human, cultural, social, and economic capital. Human capital referred to skills, knowledge, and expertise. Cultural capital designated the style of interaction with others. Social capital applied to trusting relationships and networks. Economic capital referred to material goods and resources. Construction was defined as beliefs, opinions, and attitudes about another that prompted not only the recognition of leadership qualities within the other, but also respect and deference to their judgment. Specific contexts referred to a subject area.

The participants in the study included 84 teachers from eight Chicago elementary schools. Sample selection occurred by means of selective and theoretical sampling. The researchers wanted schools designated as high poverty, urban schools with dissimilar demographics. The researchers additionally wanted schools where student achievement in math, science, or reading had improved, as well as schools where there had been no improvement. The longitudinal database of the Consortium on Chicago School Research provided the necessary information that led to selection of schools meeting the researchers' criteria. Spillane et al. (2003) conducted a 6-month pilot study with seven public elementary schools in Chicago. The formal study involved eight Chicago 
elementary schools, two of which participated in the pilot study.

Data collection consisted of interviews with 84 teachers from eight schools. Observations preceded $45 \%$ of the interviews. Interview questions focused on (a) instructional practices, (b) who or what influenced teachers' instructional practices, and (c) why these individuals wielded influence (Spillane et al., 2003). Data analysis occurred through coding interview and observation information into categories. Three criteria formed the basis of the coding process: (a) who or what influenced classroom instruction, (b) the part of instruction that was influenced, and (c) which characteristics lent legitimate authority to those who influenced instruction. Collaboration by all researchers in development of the coding categories, joint analysis of interview and observation results, and regular meetings of the researchers to achieve a common understanding of the data provided reliability.

Findings showed that $83.3 \%$ of interviewed teachers judged that principals impacted their instructional practice, while $28.6 \%$ attributed this function to assistant principals. More significantly, however, were results showing that $79.8 \%$ of teachers interviewed considered other teachers as having substantial influence on instructional practices. Spillane et al. (2003) found that teachers perceived administrators to influence instruction when cultural capital characterized their interactions with teachers. Teachers exercised influence on instruction when they interacted with other teachers utilizing cultural, human, and social capital. Teachers' construction of leadership occurred within a system of social construction. Leadership in schools must be distributed throughout an array of functions, and many individuals who have the influence of leaders are not positional leaders. Spillane concluded that cultural capital is essential for the construction 
of effective leadership, but questioned how leaders might acquire this attribute.

Rather than focusing on teacher leader interactive style as a predictor of teacher leader influence (Spillane et al., 2003), Hatch, White, and Faigenbaum (2005) conducted case studies of four teachers to examine their influence on colleagues due to levels of proficiency, integrity, and influence, regardless of holding any formal position of authority. The four teachers who participated in the study were members of the Carnegie Academy for the Scholarship of Teaching and Learning (CASTL). Each participant represented a range of different geographic contexts, different grade levels taught, different types of teacher network involvement, and different spheres of influence.

Hatch et al. (2005) found that the teachers involved in the study exerted influence on a variety of audiences at one time. That influence emanated from (a) sharing concrete ideas about classroom practice, (b) acting as teaching models for colleagues, and (c) speaking as a voice for teachers in contexts where teachers were not typically heard. Using data collected by the teachers, researchers categorized the kinds of contributions these four teachers made to the professional development of their colleagues. The first category included influence teachers had on classroom practices of their colleagues. Impacting district policies characterized the second category, while the third category consisted of influencing state and national projects through presentations and publications.

The researchers discovered that peers found the work of the teachers involved in this study significant because the work grew out of classroom practice and was then presented in formats that colleagues could understand. In addition, each of the four teachers worked to remove their recognized status as leaders from their work with 
colleagues in order to avoid giving any pre-imposed credence to their work. These teachers desired that their work remain open and public to everyone, allowing others to freely examine and improve it. This approach, they believed, modeled the attitudes with which all teachers should operate. The four teachers in the study served as bridges between their peers and external organizations. Hatch et al. (2005) concluded that this study pointed to the importance of facilitating teachers" development of inquiry skills, of involving them in learning experiences that promote representation of new ideas and insights, and providing them with opportunities to share those results with a wide audience. The researchers also construed from the study that teachers need time to reflect upon their practice in order to write and speak intuitively about the results so that other teachers may benefit. Teachers also need opportunities to network with peers in other schools to develop a better understanding of successful practice. Finally, educational organizations need to initiate opportunities which enable teacher inquiry so that valuable, reflective work might be shared with wider teacher audiences.

Hatch et al. (2005) found that teacher leaders encountered less resistance from other teachers when shared information grew out of the teacher leader's individual classroom practice. Mangin (2005) examined how teacher leaders cope with issues of teacher resistance, the strategies teacher leaders develop to gain access to classrooms, and the consequences of those efforts. The premise of the study rested on the notion that the strategies which teacher leaders used to gain access to teachers established the foundation for future interaction between teacher leaders and teachers. Mangin framed the study within the context of developing instructional leadership capacity, instructionally-focused professional development, and distributed leadership theory. The investigation used 
comparative case study methodology to review the leadership roles of 12 elementary teacher leaders. Released from classroom teaching responsibilities to work with colleagues on improving instruction, these teacher leaders were non-supervisory, schoollevel staff. Based on data from a larger study of teacher leadership, this study used results of interviews with teacher leaders and classroom teachers to focus on strategies teacher leaders used to gain access to classrooms.

Mangin (2005) used a purposive sample with selection criteria requiring formal elementary teacher leaders based in schools with low socioeconomic student populations. Five school districts in New Jersey met the sampling criteria. A representative sample of 12 teacher leaders who worked primarily to improve math instruction in 15 schools within the five districts provided the teacher leader sample. Teacher leaders and principals in the 15 schools helped with selecting the teacher participants by suggesting one typically receptive math teacher and one typically resistant math teacher from each school. Data collection with teacher leaders consisted of a pre-observation interview, a day-long observation, and a post-observation interview. Interviews were semi-structured, used open-ended questions, and were recorded for transcription. Pre-observation questions dealt with the teacher leader's work, including responsibilities, types of interactions with teachers, methods of classroom access, and factors that supported or restricted task performance. Observations sought to witness interaction processes between the teacher leader and classroom teachers. An established record-keeping guide maintained the researcher's focus during the observation. The post-observation interview allowed clarification of information gained from the observation. These methods provided data triangulation, contributing validity to the study (Creswell \& Miller, 2000). 
Mangin (2005) collected data from each of the 30 teachers using semi-structured interviews with open-ended questions. Interviews lasted 45 minutes, were audio-taped, and later transcribed. Questions centered on the kinds of interactions between teachers and teacher leaders and the teachers' perceptions of the purpose and anticipated results of the teacher leader's job. Data analysis employed matrices, memoranda and contact summary sheets to promote a systematic method for organizing and analyzing all information. The analysis focused on comparing experiences of teacher leaders in methods of negotiating classroom access and helping teachers to improve instruction. Data analysis of teacher interviews employed a computer software program. These parallel analyses provided triangulation of findings.

Mangin (2005) acknowledged that study limitations included challenges to external reliability, specifically the degree to which findings may be generalized to other settings due to contextual conditions. The study's focus on classroom access strategies also limited the findings. Other factors impacting the school setting where teacher leaders worked were not explored, nor did the study present longitudinal data required for predicting instructional outcomes. Findings showed that teachers in the study commonly used three methods to gain access to classrooms: (a) developing relationships with teachers, (b) engaging in non-threatening leadership, and (c) targeting subsets of teachers. Developing relationships included explicit self-introduction, covert self-introduction, third-party introductions, and other teachers' reports of the teacher leader's helpfulness in improving student achievement. Non-threatening leadership practices included nonintrusive assistance, reinforcement of teacher leader as a peer or an extra pair of hands to help with instruction, and other teachers' reports of the teacher leader's assistance. 
Targeting subsets of teachers included teachers perceived as in most need of help, such as new teachers, teachers who had changed grade levels, or teachers who taught in tested grades. Other teacher subsets were teachers who were highly receptive to assistance and who requested help.

Findings also indicated that administrators influenced teacher leaders' access to classrooms by setting expectations for the faculty, supporting instructional change, and offering guidance to teacher leaders. Setting faculty expectations meant communicating to the faculty the teacher leader's job responsibilities and how the teacher leader would interact with teachers. Supporting instructional change included administrators' clear expectations that teachers improve instruction and that teacher evaluation results would be tied to the level of improvement. Offering guidance meant principal direction about how teacher leaders should do their jobs and with which teachers the principal wanted the teacher leader to work. Mangin (2005) called for further research to examine how teacher leaders' relationships with teachers evolved over time, how teachers are affected by the access strategies that teacher leaders use, and the subsequent impact of teacher leadership on instructional improvement.

In a departure from previous studies about teacher leaders' gaining acceptance by colleagues and access to their classrooms, Henning (2006) investigated techniques used by teacher leaders in working on specific tasks with teachers. Henning used a case study to describe how 24 teacher leaders analyzed standardized achievement test scores from their schools in order to improve instruction that would, in turn, improve student achievement. The study participants were members of a graduate program for teacher leaders. Membership in the teacher leader program required recommendations by 
principals. All members were experienced teachers and served in various teacher leader positions in the school or district.

Data collection occurred as the 24 participants formed teams to analyze Iowa Test of Basic Skills scores for the students in their schools. Each team performed three analyses. One analysis reviewed total building scores, another compared all student subgroups within the school, and the last one scrutinized performance of one specific subject area in the school. Each team's written analysis resembled the format of a research report, with the addition of visual aids to communicate findings. Data analysis included separation of reports into one of five categories: (a) correlation, (b) disaggregation, (c) trend analysis, (d) trend analysis based on norms, and (e) trend analysis based on disaggregated data. Further categorization of reports focused on differences in research questions, the period of time covered by the analyzed data, grouping of students based on scores, and comparison of scores to national norms (Henning, 2006). Research findings showed that one fourth of the reports focused on data from 1 year. Of those six reports, three used correlation procedures and three used disaggregation strategies. The remaining 18 reports used fewer than 5 years of data to perform trend analyses. Participants noted inadequate access to student achievement data older than 4 years; thus, trend analysis might have been limited due to inadequate storing and organization of pertinent information. Lack of historical baseline data might also impede the worth of trend analysis. The researcher noted, however, that participants in this study successfully used 1 year of data using correlation and disaggregation procedures.

Additional findings revealed that use of visual displays of data provided both clarity and insight that may have otherwise been missed. Henning (2006) recommended 
that instructors in the teacher leadership program teach data manipulation and transformation strategies, i.e., histograms, charts, graphs, or frequency distribution charts. Henning further suggested that instructors emphasize that conclusions drawn from data analysis must match the statistical procedure used. These descriptive study findings finally revealed that student achievement data can be analyzed in a number of ways, including the use of strategies not used by participants in this study. Any appropriate methodology employed by teacher leaders and school administrators could inform decisions about instructional practice and school initiatives.

Studies in this section primarily focused on qualitative methods to investigate how teacher leaders act as instructional change agents. Common findings showed that teacher leaders view their primary role as helping and supporting the instructional practice of teachers in their schools (Beattie, 2002; Frost \& Durrant, 2002; Hatch et al., 2005; Little, 1995; Mangin, 2005; Smylie \& Denny, 1990; Spillane et al., 2003). Ironically, teacher leaders spent the least time working in this instructional support capacity when compared to other job responsibilities (Smylie \& Denny). Teacher leaders displayed hesitancy in challenging the norm of teacher autonomy (Smylie \& Denny; Smylie, 1992b). Other teachers sometimes presented the greatest impediment to the success of teacher leaders (Little; Smylie \& Denny). Instructional change was more likely to occur as a result of working collaboratively with another teacher rather than by working independently (Frost \& Durrant; Hatch et al.; Kohler \& Crilley, 1997; Mangin; Miller, 2002; Spillane et al.). Teacher leader transparency in attitude and instructional practice, the development of cultural and social capital, resulted in gaining respect of colleagues (Beattie; Frost \& Durrant; Hatch et al.; Mangin; Miller; Spillane et al.). The 
downside of teacher leader availability to colleagues included exhaustion, lack of time to perform necessary tasks, and an encroachment upon personal time (Beattie).

Each of these studies contributed to the understanding of the lived experience of teacher leaders who act as agents for instructional change. Ten of the eleven studies used qualitative or mixed methods to describe how teacher leaders are perceived by classroom teachers, how teacher leaders approach their multiple responsibilities, and how teacher leaders address barriers that impede their influence on teachers' classroom instruction. However, only one of the eleven presented a quantitative measure of the degree to which teacher leaders actually influence teacher behaviors.

Teacher Leadership and School Leadership Trends

The literature on educational leadership indicates that school success follows effective leadership, specifically in the person of the school principal (Harris, 2002; Waters, Marzano, \& McNulty, 2003). Descriptions of what determines effective leadership within schools range from the characteristics of the leader to the quality of the school organization (Spillane, Halverson, \& Diamond, 2004). Lambert (2003b) asserts that "how we define leadership frames how people will participate in it" (p. 4) and also that "leadership is an essential part of a professional life" (p. 4). The studies in this section address teacher leaders' perceptions of their roles, how their roles support the work of the principal, and finally, how teacher leadership manifests as distributive leadership to build leadership capacity within schools.

\section{Teacher Leader Role Perceptions}

Wasley (1991, p. 4) defined teacher leadership as the "ability to encourage colleagues to change, to do things they wouldn't ordinarily consider without the influence 
of a leader." Teacher leaders' perceptions of their abilities to influence others may impact their willingness to assume such a leadership task (Smylie, 1992b). The studies in this section discuss teacher leaders' perceptions of characteristics needed to be effective.

Vail and Redick (1993) investigated the extent to which vocational and nonvocational teachers performed selected teacher leadership behaviors and then identified predictors for the emergence of teacher leadership performance. The researchers undertook this study due to the lack of literature which specifically addressed vocational teachers' leadership behaviors. The authors used variables that the literature indicated might influence the leadership behavior of teachers to develop a teacher leadership model. The model included personal characteristics, professional characteristics, school health, teacher leadership importance, and vocational/non-vocational teacher classification as contributing factors to overall teacher leadership performance. The independent variables included personal characteristics, professional characteristics, school health, and teacher leadership importance. The dependent variable was teacher leadership performance.

Vail and Redick (1993) used a stratified random sample of 61,813 Ohio secondary teachers, 51,994 non-vocational and 9,819 vocational teachers, resulting in a random selection of 500 vocational and 500 non-vocational teachers for the study. Each participant received a pre-mailing postcard, followed by a mailed survey, and then a reminder postcard, followed by a second mailed survey, if necessary. The survey consisted of three separate parts. The Personal and Professional Characteristics Survey assessed personal and professional characteristics of participants. The Organizational Health Inventory (OHI) measured school health, and used a 4-point Likert scale with 
anchors ranging from $1=$ rarely occurs to $4=$ very frequently occurs. The reliability coefficients of the OHI were .91 from a pilot study and .93 for this study. The Teacher Leadership Scale determined teachers' perceptions of their own teacher leadership behaviors and their perceptions of the importance of teacher leadership in general. The Teacher Leadership Scale used a 4-point Likert scale with anchors ranging from $1=$ not important to $4=$ very important. Reliability coefficients for the Teacher Leadership Scale were .88 for the pilot study and .93 for this study. The researchers established content validity for the Teacher Leadership Scale through a panel of educational experts and a group of secondary teachers. Data analysis consisted of correlation and multiple regression procedures.

A revision of the model proposed by Vail and Redick (1993) specified which variables from the study significantly predicted teacher leadership performance. The perception of vocational and non-vocational teachers of the importance of teacher leadership was the most significant predictor of teacher leadership performance $\left(R^{2}=.232, p<.05\right)$. School health followed as the second highest predictor of teacher leadership activity $\left(R^{2}=.069, p<.05\right)$. Professional characteristics such as current assignment $\left(R^{2}=.028, p<.05\right)$, availability of an aide $\left(R^{2}=.007, p<.05\right)$, and number of additional roles performed $\left(R^{2}=.006, p<.05\right)$, provided the next level of influence on the likelihood of teacher leadership performance. Whether or not a teacher served in a vocational or non-vocational capacity served as a minimal predictor $\left(R^{2}=.007, p<.05\right)$ of teacher leadership performance. These variables accounted for $34.9 \%$ of the variance in teacher leadership performance.

Vail and Redick (1993) recommended that efforts to develop teacher leaders 
among vocational and non-vocational teachers should be combined since these groups were more alike than different. Administrators need to provide teachers with tasks and responsibilities perceived by teachers as meaningful and important in order to develop teacher leadership capabilities. The researchers encouraged further research on what contributes to or inhibits performance of teacher leadership activities, other predictors of teacher leadership, clarification on the concept of teacher leadership, and the use of other methodologies to investigate teacher leadership.

Similarly, to understand better the characteristics and needs of teacher leaders, LeBlanc and Shelton (1997) investigated how teacher leaders perceived themselves and others as they worked in their teacher leadership roles. The qualitative research design required data collection through interviews. A purposive sampling technique secured five participants for the study. Each participant met the required selection criteria:

(a) a teacher who had participated in leadership training, (b) a teacher identified by administrators as an exemplary teacher leader, and (c) a teacher identified by peers and colleagues as one who was respected for implementing meaningful change in a school. In addition, the participants were geographically accessible for the study and represented diversity in race, ethnicity, gender, and number of years teaching. After examining teacher leadership literature for topics related to teacher views, initial interview questions surfaced. The coordinator of the Leadership Development for Teachers course at the South Florida Center for Educational Leaders reviewed the interview questions for validity. The researchers conducted a field test of the interview questions with a randomly selected teacher leader from the participant pool. Following the field test and participant verification of resulting written transcripts and other collected data, the 
researchers produced a final revision of the interview questions. Interviews followed a semi-structured protocol and ranged in time from 1 to 2 hours at the participants' schools. The same individual conducted all of the interviews. Typing of interview notes immediately followed the interviews. The addition of comments and descriptions derived from the interview provided thick description. Within 5 days of the initial interview, and before the interview of another participant, the researchers augmented initial transcripts, thus, allowing time for reflection to uncover themes within the interview data. Each participant then reviewed the data for validation and made comments or revisions to guarantee accuracy.

LeBlanc and Shelton (1997) separately coded the pilot interview results and identified underlying themes based on their teacher leader experiences. A referential technique using a review of teacher leadership literature corroborated the initially identified themes. The addition of other categories followed, as the researchers chunked interview ideas according to the following coding criteria: content, change in meaning within the excerpt, and sensibility when removed from the rest of the excerpt. Each successive interview transcript followed this protocol. With the use of this iterative development process, the researchers could identify, collapse, or refine all categories.

Results of the study provided insight about teacher leader perceptions, feelings, leadership methods, and environmental needs. Teacher leaders need lifelong learning opportunities and positive relationships with their peers. Teacher leaders possess an enthusiasm for teaching and experience job satisfaction. Teacher leaders want opportunities to learn about multiple teaching methodologies which can affect student learning and training in developing collaborative and communication skills with teacher 
colleagues. Teacher leaders need time to negotiate the challenges of change and recognition for the work which they accomplish. Teacher leaders' need for recognition of achievement frequently conflicts with the need for affiliation and can cause jealousy, apathy, or lack of recognition among peers. These outcomes can work against the collaborative goals of teacher leadership. LeBlanc and Shelton (1997) called for further research that would identify specific ways in which teacher leaders' needs might be met while decreasing the conflict between affiliation and achievement needs. The researchers also called for further study about factors that contribute to the successes and failures of teacher leaders' collaborative endeavors.

While LeBlanc and Shelton (1997) chose to focus on teacher leaders at all levels, Birky and Ward (2002) examined the experience and individual perspectives of secondary teacher leaders and what they found meaningful in their role as leaders. The researchers undertook this study to fill the void in the literature noted by Silva et al. (2000, p.781) who suggested that "too few studies document how teachers themselves experience teacher leadership." Birky and Ward assumed that teachers must find meaning in their leadership work since the involvement of those studied was voluntary and required additional time and work.

The sample selected for the study included four teacher leaders from four different high schools and school districts. The principal and two other teachers at each of the high schools viewed the participants as teacher leaders involved in educational reform and leadership activities. Recognized as informal teacher leaders, these teachers volunteered their time in activities such as curriculum writing; school, district, or state level committees; grant writing; or making presentations to colleagues. These four 
teacher leaders held no leadership titles (Birky \& Ward, 2002). Data collection processes included surveys, interviews, and e-mail reflections. Each participant first completed a survey, followed by in-depth, open-ended, conversational interviews on three separate occasions. After each interview, the four participants e-mailed reflections on the interview to the researchers. The interviews focused on personal history, information about current work experience, and thoughts about the meaning of that experience in relationship to teacher leadership. Data analysis included development of a profile of each participant to provide a context for their experiences. Identification of recurring topics and categories followed, based on concept similarity, key words, and assignment of meaning. A computer software program provided a coding method to group similar data and identify prominent and emerging themes. Use of a variety of data sources, the employment of extensive narrative data, and a conscious effort on the part of the researcher to eliminate bias in interview protocols provided trustworthiness.

Birky and Ward (2002) identified one main theme and five sub-themes from the collected data. Each of the sub-themes described the main theme of satisfaction: enjoyment, rewards, curriculum work, colleagues, and stimulation and challenge. Teacher leaders experienced satisfaction with teacher leadership activities, which provided meaning in their professional lives. This sense of contentment came from enjoying their jobs, specifically their work with students and colleagues. Teacher leaders enjoyed writing curriculum and expected that new curriculum would improve teaching and learning. Teacher leaders felt that they received rewards from making a difference in their students' lives. Finally, the four teacher leaders indicated that teacher leadership work provided stimulation and challenge which clearly related to their personality traits. 
From these findings, the researchers raised the following questions: (a) Can a teacher be a good teacher leader and not be a good teacher? (b) Do the qualities identified about teacher leaders in this study hold true for other teachers? (c) Are teacher followers needed to follow a teacher leader's example? (d) Should all teachers be prepared to act as teacher leaders? and (e) Could the statements made by the teacher leaders in this study also be made by those who are not clearly identified as teacher leaders? Birky and Ward (2002) recommended studies to compare the role of teacher and teacher leader to answer these questions. The researchers also noted that emphasis on collaborative experiences positively affected teacher leaders in the study and called for training future teachers in collaboration skills. The study findings also included implications for teacher education, administration, and staff development. Teacher educators could benefit from understanding teacher leadership activities such as curriculum writing and collaboration. Teacher educators could subsequently better prepare teacher candidates by developing skill sets that promote collaborative behaviors. Administrators could more clearly identify characteristics and collaborative skills of future teacher leaders when selecting teachers for employment. Teacher leaders could share perceptions about curriculum and collaboration with those who plan staff development experiences.

Using a different qualitative approach, Beachum and Dentith (2004) conducted an ethnographic study to uncover teacher leaders' understandings of their leadership roles in their schools, as well as how their perceptions of this role impacted practice. Connecting these perceptions and roles would reveal insights about effective school leadership structures. In addition to structured interviews, Beachum and Dentith observed 25 teachers at five schools in one school district throughout an 8-month time period. The 
five schools consisted of two elementary schools (Pre-K through Grade 5), one middle school (Grades 5-8), one K-8 school (Pre-K through Grade 8), and one high school. Member checks of interview data afforded trustworthiness. Non-participant observations of teacher-subjects included committee work, team meetings, and large faculty meetings.

Beachum and Dentith (2004) identified three themes from the qualitative data analysis about the roles of teacher leaders, as well as the school structures that support them. Specific types of school structures and organizational patterns contributed to the success of teacher leaders. Specific practices and identities were common to all teacher leader subjects. Intentional use of outside resources and ongoing relationships with the community were universal. Strong teacher teaming, regular teacher work on relevant school issues, and the employment of teachers in quasi-administrative positions described common school structural and organizational patterns. Teacher initiation of changes in school programs or practices, experiential knowledge of administrative support and encouragement to take risks, and a strong belief that their opinions were heard and respected led to the shared realization that everyone must participate in leadership behaviors. Participants indicated that their work at school reflected their beliefs in justice, community building, and service to others. Obtaining and utilizing grant money and other types of community support forged connections between these teacher leaders' schools and the larger community.

Beachum and Dentith (2004) found that schools that actively supported teacher leaders did not separate leadership from management. Shared responsibilities in both arenas moved the culture of the schools toward greater inclusion and collaboration. The researchers suspect that these types of school structures may improve student 
performance.

The researchers reviewed in this section, with the exception of Vail and Redick (1993) used qualitative methods to determine teacher leaders' understandings of what characteristics were necessary for effective teacher leadership. Vail and Redick's quantitative study revealed that the strongest predictor of teacher leadership performance was the perception of the importance of teacher leadership activity, followed by school health, and third, by the personal characteristics of the individual teacher. These findings support Lambert's (1998) assumption that "leadership is not trait theory" (p. 8). Teacher leaders possess an enthusiasm for teaching and experience job satisfaction (Beachum \& Dentith, 2004; Birky \& Ward, 2002; LeBlanc \& Shelton, 1997). The researchers question if these characteristics are true for all teachers and if all teachers should be prepared to act as teacher leaders? Teacher leaders pursue lifelong learning opportunities and desire positive peer relationships (Birky \& Ward; LeBlanc \& Shelton). Teacher leaders want opportunities to engage in meaningful, challenging work which involves collaboration with colleagues who share the same values (Beachum \& Dentith; Birky \& Ward). Teacher leaders also desire recognition for the role they play and the unique tasks they accomplish (LeBlanc \& Shelton) which can conflict with the goal of affiliation and collaboration with colleagues. Such a contradiction moved LeBlanc and Shelton to call for additional research about factors that contribute to the success or failure of teacher leader collaboration ventures. This issue may not be problematic where the school structure places teacher leaders in quasi-administrative positions, and centers on opportunities for regular teacher-team work on relevant school issues, with administrative encouragement to take risks (Beachum \& Dentith). While the role of the principal is key 
in developing leadership within the school (Lambert, 2003b), the issue remains regarding the connection between resulting teacher leadership and student learning.

\section{The Role of the Principal}

According to Moller and Katzenmeyer (1996), teacher leaders develop at different rates. Principals promote teacher leadership by facilitating and modeling change within the school, by providing time for collaboration to occur, and by developing a supportive school culture through professional dialogue. A key factor in nurturing teacher leadership is the supportive behavior of the principal (Katzenmeyer \& Moller, 2001). Lambert (2003b) sees principal leadership as "key to development of leadership capacity" (p. 53). She previously defined leadership capacity as "broad-based, skillful participation in the work of leadership“ (Lambert, 1998, p. 12). The studies in this section address the role of the principal in teacher leader work.

Smylie and Brownlee-Conyers (1992) explored the key factors related to the development of new working relationships between teacher leaders and their principals from the perspectives of both teacher leaders and principals. This study also attempted to examine how these factors functioned in the development of teacher leader and principal relationships over multiple years. Smylie and Brownlee-Conyers determined that this topic warranted examination due to the fact that principals play a pivotal role in implementing change at the building level. Principals would be the first to support and facilitate the teacher leader position or block its development. Secondly, teachers and principals work apart from one another and their relationships are shaped by deeplyrooted standards of accountability and control. Teachers and principals act from different perspectives and use different skill-sets needed for their respective responsibilities. 
The sample for the Smylie and Brownlee-Conyers (1992) study was a midwestern suburban K-8 school district with approximately 230 teachers and 3,100 students. A district-wide teacher career enhancement program initiated teacher leadership positions as a result of a negotiated teacher contract. The purpose of the teacher leadership position was to develop new opportunities for teacher professional learning and development, recognize and reward excellence in teaching, and redesign and expand teachers' professional roles and responsibilities at the school and district levels. The final sample for this study consisted of seven teacher leaders whose roles dealt with building-level instructional leadership. Their principals also participated in the study.

Data collection occurred through individual 60- to 90-minute semi-structured, tape-recorded interviews of the teacher leaders and building principals. Questions addressed leadership activities in which both principals and teacher leaders jointly participated as well as the nature of their personal interactions. Other questions sought comparative information about the principal/teacher leader working relationship at the time of the teacher's assumption of the teacher leader position to the current working relationship. Additional questions probed the factors which influenced the development of their working relationship, the strategies used to shape the working relationship, and turning points that led to the current status of their working relationship. Finally, all participants responded to questions about the impact of identified turning points in the principal/teacher leader working relationship. Data analysis made use of the constant comparative method of content analysis. This method allowed identification and classification of data themes across data from all participants. Smylie and BrownleeConyers (1992) established validity by presenting and discussing the identified themes 
with two independent university researchers who had no affiliation with the study but who were experts in educational leadership. The researchers next conducted member checks by sharing themes with all study participants as well as a district-level administrator who acted as a participant observer for the implementation of the district teacher leader program. The authors of the study incorporated feedback from these three groups into the study.

Results of the Smylie and Brownlee-Conyers (1992) study reflected strategic, interactive, and contextual elements which impacted the development of both the teacher leader initiative and the subsequent development of principal/teacher leader working relationships. First, ambiguities and uncertainties associated with new teacher leader roles had significant implications for changing working relationships between principals and new teacher leaders. The greater the perceived ambiguities, the more likely that principals and teacher leaders tried to formalize definitions and routines related to their roles and relationships. Second, the directions in which principals and teacher leaders tried to define their roles were strongly slanted by the perceptions about the role that each individual brought to the table. Principals and teacher leaders shaped new roles according to their own perspectives. Third, principals and teacher leaders used strategies that influenced the development of the new roles and working relationships. These strategies, whether formal, planned meetings, or unplanned conversations, shaped both the tasks and the interpersonal elements of the working relationship. Fourth, events not directly associated with daily work played significant roles in the development of new working relationships between principals and teacher leaders. Outside events could accelerate development of new relationships or simply reinforce use of normal relations. Fifth, 
development of new working relationships between principals and teacher leaders could involve a move from internal, self-interest to mutual interdependence focused on school improvement. The researchers concluded the most important implication of the research findings was that the success of principal/teacher leader relationships related to teacher work re-design was not just the structure of new roles for teachers, but the development of the interpersonal elements of their working relationships. Teachers and principals needed to resolve interpersonal tensions and establish trust, as well as develop a method for effective communication so new roles could function effectively. Districts should work proactively to foster interpersonal knowledge and skill needed for principals and teacher leaders to work together successfully.

Negotiation of principal/teacher leader roles framed other studies. Hart (1994) conducted a comparative case study of two schools engaged in career ladder reform to examine organizational change in the form of teacher work design. The goal of the analysis was to learn from the people involved directly in the reform, to review their evaluation of the important values and activities in their schools as impacted by a change in teaching and leadership roles. Comparative case study methodology provided opportunities to identify differences in the experiences of similar groups. The sample for the study included volunteer faculty members from two junior high schools implementing the teacher career ladder program in a single school district. Data collection included systematic field notes collected through non-participant observation for 2 days a month at each school from September through May and structured and unstructured interviews. The researcher conducted a total of 164 structured interviews with teachers (career ladder participants, non-participants, and novice teachers), principals, and assistant principals in 
three cycles during the school year. Interviews ranged from 30 to 60 minutes in length, were tape-recorded and transcribed. Field notes chronicled data from informal, unstructured interviews with students, teachers, and administrators throughout the year. Other data consisted of surveys, audiotape transcripts from meetings the researcher did not attend, teacher journals, notes, and transcripts of post-observation conferences between teacher leaders and novice teachers and between teachers and the principal. Additional documents reviewed but not created for the study included the career ladder plan, job descriptions for each school, newsletters, in-service training materials for career-ladder teachers, official minutes and teachers' notes of task-force and faculty meetings, and newspaper articles related to career ladder events.

To moderate investigator effects, a teacher at each school watched for behavior changes that occurred during or after observations or interviews. Teachers and administrators named one experienced, respected teacher at each school who opposed the career ladder structure and did not participate in the career ladder program. Hart (1994) conducted formal interviews and numerous informal conversations with these two teachers. The researcher also interviewed unsuccessful applicants for the career ladder program and members of the selection committee at each school. Subsequently Hart conducted member checks of conclusions with teachers and administrators after finishing the data-gathering phase of the study. Data analysis consisted of coding all transcript data, journals, and field notes using role theory concepts. Gathering quotations and field notations into data summary sheets provided comprehensive collections of specific examples of a role theory concept evident in the raw data. Summary sheets also indicated the role associated with the quotes, i.e., teacher, teacher leader, principal. Comparison 
matrices facilitated the analysis of interaction patterns. The matrices compared role theory occurrences by frequency, time, school, and position, revealing information about perceptions about role-specific performance effects.

Three components of role theory provided the theoretical framework for Hart's (1994) work. These components included (a) roles existing as identities within systems, (b) the enforcement and evolution of roles, and (c) the social processes that constructed the interpretations and shared reality of the study participants. Data analysis based on these criteria revealed career ladder jobs as a set of new positions within the school social system, rather than as adjustments in the structure of existing teacher roles. The role and function of teachers became unclear during the career ladder implementation process, complicated by disagreement over whether the career ladder fundamentally restructured the role of teachers or whether it modified teacher job categories. Administrators and teachers formed group alliances in favor of or against the career ladder change that defined teacher work.

Teachers' professional identities influenced teachers' and administrators' reactions to new career ladder positions in both schools in the study. While the principal and teacher leaders at one school worked together to define their roles, advertise their activities, and openly deal with questions from the faculty, the teachers in career ladder positions at the other school did not coalesce into a core group with a published set of job responsibilities and goals. The principal in the first school set up opportunities for career ladder teacher leaders to exercise leadership, while the principal in the second school was silent about the role of the teacher leaders, and left those individual teachers with no systemic support (Hart, 1994). Role theory suggests that the enforcement of roles can be 
powerful forces in stable organizations. Thus, the values and norms of each school in the study influenced how teacher leaders in the career ladder program exerted influence. In practice, administrators and teachers at the first school transformed the career ladder program from teacher promotion and incentive to staff development and a school improvement initiative. The latter design matched the existing beliefs and practices of the building faculty. In the second school, career ladder teacher leaders met critical resistance from teachers and silence from the principal.

Hart (1994) concluded that leaders must attend to individual teacher's roles and the faculty's assessment and review of those roles. The attention and support of each principal significantly contributed to the importance with which teachers viewed those in teacher leadership positions. Different signals from the two principals in the study produced significantly different results among faculty members' perceptions about the importance, professional authority, and acceptance of teacher leaders. Principals needed to offer visible and frequent reinforcement of teacher leader work and attend to the social-emotional adjustments required by leadership. In this study, the pressure to implement change collided with the pressure to resist change. One school successfully facilitated faculty growth and development. The other school could not negotiate the uncertainty and discomfort of change. The difference between the two outcomes rested with a core support group of a well-functioning principal and teacher leaders.

In a separate investigation, Brown and Anfara (2002) directed an exploratory qualitative study which was part of a larger study on middle school principals. The purpose of Brown and Anfara's work was to shed light on collaborative efforts between administrators and teachers in order to improve teaching and learning. The participants 
solicited for the study included 125 Pennsylvania and New Jersey middle school principals and yielded a response rate of $60 \%$. Data collection consisted of gathering survey data and conducting semi-structured interviews. Surveys posed both open- and closed-ended questions concerning the participants' (a) educational, professional, and personal background; (b) understanding of middle school philosophy; (c) school reform and change agent experience; (d) views regarding parent involvement in school, and (e) understanding of special education matters. Closed-ended survey responses were scaled and described descriptively. Open-ended response questions were categorized by common themes. Selection of 17 survey participants who characterized the larger sample followed. These 17 principals participated in the interviews, which were tape-recorded and then transcribed. Data analysis included numerous re-readings of transcripts to identify persistent themes, coding of transcript information, and computation of survey data. Triangulation of interview and survey data, use of precise quotes, maintenance of an audit trail, and member checks assured internal validity.

Brown and Anfara (2002) concluded that the study participants believed administrators must engage in specific behaviors in order to overcome walls of division that separate teachers and administrators so that focus can be maintained on teaching and learning. Principals must (a) be present and available to teachers, (b) actively and attentively listen to staff ideas, (c) create a positive school climate through appreciation and support, (d) collaborate and share decision making, and (d) manifest their passion for their students by inspiring others to that same enthusiasm. Brown and Anfara concluded that leadership has become more reliant on task competency rather than formal position, and because of that evolution, principals must identify, nurture, and rely upon the vast 
competencies of the teachers within their schools.

Andersen (2004) conducted a multi-site case study to contribute to a better understanding of the nature of teacher leadership and how teacher leaders interacted with principals. The definition of teacher leadership employed by Andersen in this study consisted of setting directions as well as influencing others to move in those directions. Teacher leadership implied mutual influence between teacher leader and teacher follower and could be exercised formally or informally. Six Canadian schools participated in the study, based upon shared criteria of an active school improvement process and location in the same general geographic region. With the exception of one school, five people from each school agreed to be interviewed. These individuals consisted of two teacher leaders, two teachers who named peers as being teacher leaders, and the principal. Only three individuals participated in one school, one from each category, due to low staff numbers. Formal teacher leadership roles did not exist in the majority of the participating schools. The interview questions used in the study emanated from previous research on the nature of teacher leadership, providing previously established reliability for the instruments. Data analysis consisted of coding and counting frequency of ideas offered through interviews. Frequency data provided a discussion focus that assisted in shrinking the amount of data to a workable size as well as upholding the integrity of each participant's perspective.

Andersen (2004) found that all participants recognized that teacher leaders exerted influence on principals. Significantly greater influence of principals on teacher leaders also emerged from the data. However, leadership reciprocity became overwhelmingly evident from the study respondents. Three models of influence between 
principals and teacher leaders became apparent from the data.

The Buffered Model placed the principal in the center, surrounded by teacher leaders who isolate and insulate the principal from other teachers and outside pressures. This model appeared to Andersen (2004) to limit the widening of teacher leader influence to informal teacher leaders. The Interactive Model witnessed the principal interacting with all staff, as well as distributing decision making among all teachers. In this model, informal and formal teacher leaders were interrelated, and the principal was available and visible to all. Transformational leadership blossomed in the Interactive Model. The Contested Model positioned the principal outside the circle of influence and interaction, in opposition to teacher leaders. The dominant-dependent paradox between principals and formal teacher leaders pitted the two entities against each other and brought progress to a standstill. Andersen concluded that formal teacher leadership, such as department head positions, both hindered and prohibited the development of other forms of teacher leadership. Collegiality was more common in the participating schools where formal teacher leadership positions did not exist.

In a separate study, Foster (2004) investigated the relationship between secondary school leadership and successful schooling through the perspectives of principals and other school members. Two research questions framed the study. How do school members (principals, teachers, students, and parents) in secondary schools which have implemented school improvement plans construct the concept and practice of leadership? How do these school members perceive and understand the relationship between leadership and school improvement?

Foster (2004) used a case study design because of its constructivist assumptions: 
(a) there are multiple ways of viewing and interpreting reality; (b) individuals know and understand reality through their interactions with others; and (c) knowledge is socially constructed. Conducted over 8 months and in three stages, the study involved two secondary schools in western Canada. Phases 1 and 2 consisted of data collection, analysis and report writing in the first and then the second school. Phase 3 included analysis of themes that emerged from the data gathered in Phases 1 and 2. The two secondary schools in the study possessed populations ranging from 1,000 to 1,200 students. Both participated in multi-year school reform initiatives and enjoyed positive reputations among school and community members.

Foster (2004) collected data through individual and focus group interviews, classroom observations, and document analysis. Those who participated in the individual interviews $(N=31)$ also participated in the focus group interviews. Participants from each school included the principal, assistant principals, six to seven teachers, four students, and two parents. Purposive sampling, based on diversity of perspectives, gender balance, and student participation in Grades 10-12 determined individual participants in each school. Each individual in the study participated in two, independent, 30-minute interviews. The four different focus groups in each school (principals, teachers, parents, and students) participated in two, independent interviews. The first focus group interviews preceded the initial individual interviews, and the second focus group interview followed the conclusion of all individual interviews. All interviews were taperecorded and transcribed; copies were then given to participants for member checking. Data analysis consisted of multiple reviews of field notes, documents, and written transcriptions of interviews. Use of the constant comparative method to code all data led 
to identification of emergent themes. Member and expert checks helped confirm interpretations and enhance trustworthiness of findings.

Findings of the study indicated that leadership in schools focused on improving student learning comes from varied sources, should not be equated with the principal or others with designated leadership roles, and is a shared social influence process. Principals and teachers believed that they shared responsibility and influence in goal setting for improving student learning. Teachers believed teacher collaboration and continuous professional learning were critical for developing leadership for school success. Overall, students and parents were excluded from participating in the leadership process for school improvement, viewing themselves as consultants who could offer opinions only when asked. Foster (2004) called for further research that adopted a more pluralistic approach in uncovering diverse perspectives and social interactions within the school context. She encouraged future researchers to adopt approaches which recognize leadership varies from place to place. This insight could help address blank spots in understanding the role of leadership in school improvement.

Studies in this section were all qualitative, examining the nature of teacher leadership, the interactions and relationships of teacher leaders with their building principals, (Andersen, 2004; Smylie \& Brownlee-Conyers, 1992), as well as the nature of collaboration between principals and teacher leaders (Brown \& Anfara, 2002; Hart, 1994). Findings about the principal/teacher leadership relationship showed that the greater the level of perceived ambiguities in a new teacher leader role, the more likely that principals and teacher leaders would try to define their roles formally (Smylie \& Brownlee-Conyers). Principals and teacher leaders shaped the new teacher leader roles 
according to their own perceptions (Brown \& Anfara; Hart; Smylie \& BrownleeConyers). Outside events played important roles in the development of new working relationships between principals and teacher leaders (Smylie \& Brownlee-Conyers). Development of new working relationships between principals and teacher leaders meant moving from independent behaviors to interdependent behaviors focused on school improvement (Foster, 2004; Hart; Smylie \& Brownlee-Conyers), although the nature of school improvement was not defined. Development of the interpersonal elements of the working relationship between principals and teacher leaders proved to be the leading factor in predicting the success of the teacher leader position (Brown \& Anfara; Smylie \& Brownlee-Conyers). While principals influenced teacher leaders more than teacher leaders influenced principals (Andersen), findings showed principals relied upon the skills of the teachers within their schools to maintain focus on teaching and learning (Brown \& Anfara). Principals who effectively supported teacher leadership paid attention to the teacher leader's role as well as the faculty's perception of that role. Additionally, these principals offered frequent, visible reinforcement of teacher leader work to support collaborative efforts (Hart).

This section also addressed the role of the principal with teacher leaders where multiple teachers acted as leaders (Andersen, 2004; Brown \& Anfara, 2002; Foster, 2004). Interestingly, collegiality was more common in schools where formal teacher leader positions did not exist (Andersen). Schools where effective principal leadership led to leadership as a shared social influence process saw the principal and all teachers sharing responsibility and influence to improve student learning. Teachers in this setting believed teacher collaboration and continuous professional learning were of highest 
importance for school success (Foster). These results raise questions about where more significant impact on teaching and learning might occur: with one building teacher leader who works with the principal; with one building teacher leader who supports the principal in developing collaborative teams of teacher leaders within the school; or with the principal developing effective collaborative teams throughout the school without benefit of a formal teacher leader? How effective are the interactions of teacher leaders with teachers in improving teaching, learning, and student achievement?

Teacher Leadership and Distributed Leadership

Distributed leadership theory proposed that schools share leadership in a more democratic and collective form of leadership (Gronn, 2002; Muijs \& Harris, 2003). Moller and Katzenmeyer (1996) defined teacher leaders as those who "contribute to school reform or student learning (within or beyond the classroom), influencing others to improve their professional practice, or identifying and contributing to a community of leaders" (p. 5). According to Muijs and Harris, distributed leadership clarified the work of teacher leadership for three reasons. First, distributed leadership addressed the work of multiple groups within a school who guide instructional growth. Second, distributed leadership suggested social distribution of leadership where leadership tasks were stretched over the work of many persons and where the task of leadership was achieved through the interaction of multiple leaders. Third, distributed leadership involved interdependence rather than dependence. The emphasis of teacher leadership upon collaborative action and empowerment of all toward accomplishment of a shared goal reflected distributed leadership theory (Muijs \& Harris). The studies in this section address teacher leadership as an enactment of distributed leadership through planned 
change and shared responsibility for school improvement.

Heller and Firestone (1995) studied the sources of leadership for planned change in eight elementary schools. With the help of the Social Problem Solving (SPS) Organization, the researchers identified eight schools in the institutionalization stage of implementing the SPS program. The SPS program teaches skills that assist students in problem solving and decision making to support successful social behaviors and interpersonal relationships. The institutionalization stage follows the adoption and implementation stages and occurs 1 to 2 years after adoption. Institutionalization marks the point when the practice becomes a normal part of life.

In this study, Heller and Firestone (1995) sought to balance the number of schools that experienced complete or partial success in operationalizing the SPS program. Success meant that both SPS experts and researcher interviews of school personnel indicated reliable program implementation. The participant sample included seven average socioeconomic, suburban elementary schools with minimal student diversity, and one very poor, inner city elementary school. The data collection tools included structured, open-ended interview questions to investigate three areas. The first queried the level of staff perception of the success of SPS implementation. The second addressed school-wide leadership supporting change required for SPS success. The third assessed the contributions key position holders in the school made to the first and second areas of staff perceptions and school leadership for change. Pre-testing of interview questions occurred twice with schools and principals employing the SPS program to assure clarity of language.

Heller and Firestone (1995) conducted interviews of each principal and three 
teachers selected by the principal. An additional interview occurred with SPS specialists assigned to each district plus a district level employee who served as the SPS supervisor. Researchers conducted 42 interviews over a 4-month period. Teacher interviews lasted between 30 and 40 minutes, depending upon the length of planning periods. All other interviews consumed longer periods of time based on subject availability. Data analysis included original verification of institutionalization of the SPS program through SPS experts and later, through teacher interviews about the SPS curriculum, teachers' weekly SPS lessons, and teachers' observations of SPS impact on student behaviors. Teacher interviews served as a means of data triangulation and verified initial SPS specialists' evaluations of institutionalization. Next steps in data analysis included review of principal, district supervisor, and SPS district consultants' interviews. Finally, interview data ascertained which specific job positions performed tasks that led to the success of the SPS program. Data triangulation within school positions, as well as across schools, improved accuracy.

The outcomes of the study indicated functions supporting program institutionalization included sustaining a clear vision for change, providing formal and informal encouragement, and monitoring the progress of the program. Assurance of adequate resources and adjustments of school schedules and procedures also enhance institutionalization. No one person was identified as the sole facilitator for program change. On the contrary, data indicated that redundant leadership roles for change existed in the participating schools. Many individuals who held different positions in the school acted as change leaders. Researchers noted task redundancy among the multiple leadership roles as well. Teachers assumed active roles in sustaining the vision of the SPS 
program, monitoring its progress, and providing encouragement to one another. According to study findings, Heller and Firestone (1995) concluded teacher leadership can work in current school organizational structures, playing a significant role in supporting other leadership positions. Teacher leadership can provide leadership for change through shared redundant tasks. Redundancy of shared tasks connoted performance of the same function by many people in different roles and provides mutual reinforcement of the desired change. Redundancy of shared tasks assured attention to critical issues.

In a separate investigation, Whitaker (1997) conducted a descriptive case study to examine how teacher leaders and other teachers and staff members viewed the teacher leader management team model. The study investigated (a) how teacher leaders viewed their positions, (b) how teacher colleagues viewed individuals who held teacher leader positions, (c) the advantages and disadvantages of the teacher leader construct as interpreted by teachers and other management team members, and (d) insights regarding the role the principal played with teacher leaders in the management team model in the early phases of implementation.

The unique case selection process, based upon a distinctive characteristic intrinsic to the population, guided selection of the school site. The 2-year study involved five teacher leaders, 31 staff members, and the principal in one elementary school. Data collection consisted of interviews, observations, and document mining. During the first year of the study, Whitaker (1997) conducted semi-structured interviews with 13 teachers. Criteria for interview selection included a representation of different grade levels and number of years' teaching experience. The researcher shared interview data 
with participants to check for accuracy. During the second year of the study, Whitaker conducted another round of interviews with the school secretary, 13 teachers who represented each grade level and various years of teaching experience, the teacher leaders, and the principal. Whitaker observed management team meetings and faculty meetings, as well as how management team members executed leadership tasks. The researcher categorized data from interviews, observations, documents, and field notes into categories.

The findings revealed that faculty members approved of the idea of teacher leaders serving on the school management team. Faculty members viewed teacher leader roles as helpful to the school and endorsed a collaborative leadership style. Data showed communication issues existed between faculty and management team members and that teacher leader roles lacked clarity. Teacher leaders handled more administrative duties rather than the curricular and instructional responsibilities which they anticipated and wanted to manage. Failure to concentrate teacher leaders on instructional concerns was due to the principal's lack of foresight and understanding of curricular primacy. The absence of a pre-existing, trusting relationship between the principal and the management team inhibited the success of the management team model. Whitaker (1997) concluded that principals need knowledge and understanding of organizational change models, as well as good communication skills, in order to lead teachers successfully into the collaborative structures of shared leadership.

In another school reform study, Datnow and Castellano (2001) conducted a qualitative investigation of leadership in Success for All (SFA) schools. SFA was a research-based whole-school reform model which organized resources to ensure that 
students succeeded in reading in elementary school. SFA took an aggressive stance in changing teaching and learning. The program was highly specific in implementation guidelines and materials for students and teachers. Ongoing teacher professional development supported program implementation. The principal and school facilitators were critical factors for SFA success because the program demanded substantial administrative support and monitoring. SFA called for a strong school-based teacherfacilitator, recognizing that a principal cannot manage reform alone and that the best support for teachers was a teacher colleague. Because SFA focused on the details of teaching and learning, the reform initiative provided an opportunity to evaluate how leadership improved both teaching and learning.

The sample for the Datnow and Castellano (2001) study consisted of six elementary schools which used the SFA program, three in different school districts in California, and three in the same district in the southeastern United States. All schools in the study served primarily low-income students. Principals in the study were both new and experienced, while teacher-facilitators were all former teachers with 15 or more years of teaching experience. Data collection occurred through interviews with principals, teacher-facilitators and teachers, either individually or in small focus groups. The researchers conducted a total of 103 interviews over a 2-year period. Teacher interviews took approximately 45 minutes, while interviews with teacher-facilitators and principals averaged 1 hour or longer. The authors elected to conduct informal interviews with school staff members. All interviews used semi-structured protocols and were taperecorded and transcribed.

Data analysis occurred in several steps. The researchers coded interview 
transcripts with the purpose of the study in mind, first using an open coding method. Open coding referred to the process where concepts were identified and developed through asking questions about the data and then labeling and grouping similar events and incidents into categories. Axial coding followed open coding. Axial coding referred to grouping the data from the open coding process into more specific categories. The next step involved reducing the data within some codes to a series of matrices to conduct within and across-school analysis. The researchers recorded operational notes during the coding processes. These notes documented observations of code descriptions and issues that emerged from the coding. These operational notes prevented researcher bias and logged ongoing records of the data analysis process.

Datnow and Castellano (2001) found that the leadership of the principal served as a primary factor in adoption of the SFA reform model. Once in operation, the SFA program shaped the role of the principal and the principal's leadership style shaped the implementation of the SFA model. The most significant role change for principals due to SFA implementation was a more precise focus on teaching and learning. SFA gave principals a new opportunity to become knowledgeable about classroom reading instruction. SFA teacher-facilitators provided a critical leadership position for program success. Teacher-facilitator roles included monitoring program implementation, teacher and student progress, student assessment, and teacher training. The researchers observed that the SFA teacher-facilitator role demonstrated less ambiguity than teacher leader roles in other contexts. SFA teacher-facilitators operated under clear job descriptions which required extensive time, effort, and management of multiple, defined functions. Ironically, the researchers found that SFA teacher-facilitators endured levels of role 
ambiguity. The SFA teacher-facilitator position fit neither the role of administrator or teacher, but some combination of the two. Because of this uncertainty, teacher-facilitators experienced conflict with principals and with teachers. While the teacher-facilitators worked at building trust with both groups, the relationships required on-going work. These findings about the tensions in the role of teacher-facilitator reflect the literature about teacher leadership.

Implications for the study suggested that the SFA model could focus school administrators on school improvement through effective reading instruction. One downside of the model included principal and teacher-facilitator monitoring teacher implementation of the SFA curriculum. This monitoring process placed teacherfacilitators in an evaluative role and teachers in a compliance role, limiting shared leadership at the classroom level. SFA called principals to more managerial and administrative leadership instead of creative or inclusive leadership. The researchers clearly believed one teacher-facilitator per school was not enough to bring teachers and administrators together in shared leadership. Datnow and Castellano (2001) recommended further research of the change in relationships between teachers and administrators and among teacher-roles intrinsic to other school reform models.

A study published the following year presented just such research data about relationships between teachers and administrators as well as among teachers. Chrispeels and Martin (2002) examined the roles and responsibilities of school leadership teams as well as how perceptions of their place in the organization influenced their roles. The researchers also investigated the types of actions the leadership teams took in support of student achievement and in the overall organizational structure of the school. This study 
centered on the concept that leadership teams would have a greater positive effect on student outcomes over the impact of a single teacher leader. Selection of the sample was purposive, consisting of school leadership teams from four middle schools considered representative of middle school teams who participated in the California School Leadership Team Professional Development Program (CSLA). The CSLA viewed these four schools as making progress in implementing program goals. The four schools represented three school districts and three geographical areas of California. Three schools were urban and one was suburban. All schools represented ethnically diverse student populations.

Data collection included interviews, observations, and video data from two training sessions, as well as surveys. Interviews consisted of semi-structured, open-ended questions which explored the roles each team assumed at their school, the activities of the team, and the challenges the team faced. Interviews with each team member lasted from 30 to 60 minutes and were tape-recorded and transcribed. Chrispeels and Martin (2002) reviewed each school's organizational chart to determine how the school leadership team related to the total school structure. Observation of video data from school leadership training sessions offered understanding of the range of topics addressed, as well as insights about how the leadership team functioned and the relationships between the principal and team members. Data analysis consisted of independent review of all transcripts by three researchers connected with the study. After each researcher identified major themes, the three researchers compared and discussed the themes for agreement. Organizational theory, systems theory and micro-political theory provided an additional interpretive framework. Coding and tabulation of interview data preceded chart design to 
identify specific categories of team interaction, roles, relationships, team function, and team power and influence regarding school decisions. The researchers drew conclusions based on the charts and verified findings from the interviews.

Findings from the Chrispeels and Martin (2002) study revealed that all participating schools had a multi-committee structure when the school leadership team program began. Designing a school organizational chart helped leadership teams from each school identify patterns of power, communication flow, and social relationships. Each leadership team assumed responsibility for communication, staff development, problem solving, and decision making. The School Leadership Team seminars provided significant opportunities for teacher and principal collaboration, which laid the groundwork for teachers to become competent reform leaders. The training decreased teacher isolation and brought teacher leaders together as an energized, focused learning community. The training also surfaced organizational and political conditions which leadership team members faced. Organizational learning became an important factor for establishing their role as a school leadership team. Gaining knowledge about the school organization gave each team a source of expert power and the potential influence to shape school decisions. Each team took action and exerted influence on their teaching colleagues as well as on school decisions, many of which impacted teaching and learning. Each team engaged in a continuous process of defining themselves during the 3-year training period. The teams' opportunities to use expert power flowed from positional power. Teams that gained authoritative position in the school after 3 years maximized their expert power to influence teaching and learning.

Chrispeels and Martin (2002) suggested that the findings had implications for 
policymakers and educational leaders. First, development of new structures such as school leadership teams could be the catalyst for changing power and authority relationships. These changes should be followed over time to understand their genuine impact on the organization and on student learning. Second, school leadership teams need knowledge of existing organizational structures, rules and relationships, and an understanding of how to negotiate a place within the system. Context mattered. Third, training gave school leadership teams the authority to act as school leaders and the knowledge and skills to work with their schools. Fourth, there was no set formula for understanding how a new structure within the school, such as a school leadership team, would be situated within the school. Fifth, teams must learn how to read the political dynamics of their schools and engage in organizational learning in order to assume leadership and develop qualities needed to renew existing school structures. Changes in relationships and behavior patterns took time. Finally, the process of preparing teacher leadership teams required time and active direction and support. Organizational reconfiguration and change in role relationships were difficult undertakings.

The impact of school context on distributed leadership structures and processes presented another question. Harris (2002) conducted qualitative case studies of 10 schools facing challenging circumstances in England. Challenging circumstances included having $25 \%$ or fewer students in the school passing an external exam at 16 , or $35 \%$ of students receiving free meals. Two criteria determined the 10 schools selected for the study: (a) representation of a wide range of geographic, socio-economic, and cultural populations; and, (b) student achievement trend data and inspection reports that confirmed successful leadership. The purpose of the study was to contribute to the 
research base about successful leadership practices within difficult school contexts. Data collection took place through interviews and mining of data. The researchers conducted 50 semi-structured interviews with head teachers, middle managers, and classroom teachers across the 10 participating schools. Interviewees reviewed transcripts for correction and validation. Researchers also collected and analyzed a wide range of documents and contextual data from each participating school. Initial reporting of withincase analysis preceded between-case analysis and checking of findings with head teachers from a group of schools facing challenging contexts but not participating in the study. The aim of these procedures was to present a thick description of leadership practices within the 10 participant schools and the extent to which the specific school context shaped leadership practices.

Harris (2002) found that leadership approaches varied depending upon the stage of the school's development. These school leaders eventually moved from an autocratic leadership style to one that empowered others in the school to lead. Various forms of teacher leadership were common in the 10 schools studied. This form of distributed leadership impacted problem solving and decision making within the schools. Equity and empowerment emerged as hallmarks of leadership in these improving schools with challenging contexts. Instructional innovation and risk taking were laudable actions. Individual and staff development opportunities, involvement in decision making, and granting professional autonomy to teachers facilitated the building of trusting relationships within the school community and shared ownership of the school's mission. An emphasis on people more than systems characterized the invitational style of leadership evident in the participant schools and facilitated their movement toward 
professional learning communities. Effective leadership meant building leadership capacity in others in order to engage all teachers in the school. The clear focus on improving students' academic achievement provided the common vision that unified the school communities. This focus guided school leaders to understand that if learning opportunities for students were to improve, then opportunities for teachers to grow professionally and develop and share expertise also must improve.

In another school reform study, Camburn, Rowan, and Taylor (2003) analyzed survey data gathered from samples of elementary school leaders during the second year of participation in the Study of Instructional Improvement (STI). The Study of Instructional Improvement housed an ongoing research study about the design, implementation, and instructional effectiveness of three of the largest and most widely implemented comprehensive school reform (CSR) models in the United States, namely, the Accelerated Schools Project (ASP), America's Choice (AC), and Success for All (SFA). Camburn et al. used data from the second year of the study to examine how implementation of the CSR model affected the leadership activities of those who occupied roles generally charged with exercising leadership in elementary schools. These roles included principal, assistant principal, program coordinator, and other leadership positions created by the specific comprehensive school reform model, such as instructional coach or facilitator. Independent variables consisted of three forms of comprehensive schools reform, namely, ASP, AC and SFA. The dependent variable was leadership activities of those charged with leadership roles in elementary schools.

Camburn et al. (2003) addressed three questions. (1) Did the CSR schools have a greater number of formally-designated leadership positions than schools that did not 
participate in the CSR program? (2) How were leadership functions distributed across the formally-designated leadership positions in the CSR schools? (3) Did schools in the CSR programs display the kind of widely distributed and redundant pattern of instructional leadership that previous research on distributed leadership suggested for successful program change and instructional improvement? Participant selection occurred in four steps. The researchers first compiled a list of all public schools in the United States affiliated with ASP, AC, or SFA in the 1998-1999, 1999-2000, or 2000-2001 academic year. Schools were divided into 17 geographic regions for selection purposes. The researchers attempted to equalize school samples based on the length of affiliation with one of the three CSR programs, and according to socioeconomic disadvantage. Researchers also selected a set of comparison schools from the 17 geographical regions. Participant schools selected for the study included 114 schools, in 45 different school districts, in 15 different states, and in 17 different metropolitan areas.

Two instruments, the School Leader Questionnaire (SLQ) and the School Characteristics Inventory (SCI), facilitated data gathering. The SLQ measured instructional leadership, building management functions, and boundary spanning functions with alpha reliabilities of 0.77 to 0.90 . The SCI recorded data on the structural features of schools, including the full-time equivalent appointments in various formally designated positions in schools. Five hundred and three elementary school leaders received the SLQ and returned responses at a rate of $88 \%$. Principals from all 114 schools received the $\mathrm{SCI}$ and $96 \%$ responded. Camburn et al. (2003) used multiple regression to examine leadership configurations and whether or not schools with CSR models used different leadership configurations than non-CSR schools. Results indicated that larger 
schools and schools which served more disadvantaged populations generally had larger administrative staffs. Schools which implemented one of the three CSR models had a proportionally greater number of leadership positions than the comparison schools. In addition, schools which implemented any of the three CSR models allocated more personnel resources to subject area and program coordinators than did other schools.

The second level of data analysis addressed how time spent engaged in instructional leadership functions varied within and across schools. Principals, assistant principals, and CSR staff reported addressing above average priority to instructional leadership, indicating that leadership was distributed across these roles redundantly, as well as possible specialization among leadership group members. Coaches in CSR schools reported giving more time and priority to instructional leadership than leaders in other positions outside of principals and assistant principals. CSR programs appeared to impact the distribution of leadership by re-configuring the size and composition of leadership. In addition, CSR participation generally affected the amount and kind of leadership functions performed, leadership functions directly supportive of instructional growth (Camburn et al., 2003).

The CSR "coach" position was the most significant factor in observed levels of instructional leadership. The primary function of the instructional coach was to develop instructional capacity within the school. Leaders who engaged in extensive professional development increased reflection upon instructional practice as well as levels of instructional leadership. CSR processes clearly identified instructional leadership roles and provided extensive training in instructional leadership practices. These leadership processes appeared to be associated with higher levels of instructional leadership. Thus, 
active communication of expectations through staff development could encourage instructional leadership practice rather than more passive methods of role definition and role description. Camburn et al. (2003) noted limitations of the study. All information on leaders' activities came from self-reported data and represented a cross-section of participant schools. Longitudinal observations of changes in leadership activities would provide a deeper, more accurate understanding of how CSR programs reconfigured leadership structures.

Turnbull and Mee (2003) examined the concerns, issues, and ideas of school personnel directly involved in school-based management teams, both team members and non-team members. The researchers sought to examine management team member feedback from the perspectives of those required to monitor team progress or assist teams with self-review and evaluation processes. The sample for the study consisted of 31 property-poor districts in New Jersey who received a government mandate to implement a school management team (SMT). The purpose of the SMT was to guarantee participation of school staff members, parents, and the local community in school-level decision making, as well as to develop a culture of cooperation, accountability, and commitment among all stakeholders to improve student achievement. The primary responsibility of each SMT included development of a total school reform implementation plan based on a school-wide needs assessment. Seventy-two schools, referred to as Cohort One, implemented SMT during the 1998-1999 school year. Another 83 schools, referred to as Cohort Two, began SMT at the beginning of the 1999-2000 school year. The sample for the study consisted of teachers in 55 property-poor schools from six school districts in New Jersey from Cohorts One and Two. Each of the six 
districts volunteered to participate in the study.

Data collection occurred through administration of a teacher questionnaire which included three open-ended questions: (a) How would you describe your involvement in school-level decision making? (b) In what types of decisions or areas of decision-making have you been involved so far this year? and (c) Are you satisfied with your level of involvement in school-level decisions? The questionnaire asked if the participant was a member of the SMT and the number of years of teaching experience. Turnbull and Mee (2003) initially distributed the questionnaires at school site visits and sent follow-up letters with another questionnaire and a postage-paid envelope to those who did not respond. Participants received three rounds of questionnaires each year of the 2-year study. The response rate for the first year was $53 \%(N=670)$. During the second year, only $58 \%(N=384)$ of the first year's $53 \%$ returned useable responses. Data analysis began with organizing information in a nested manner so that responses could be examined by item, by teacher, and by school. Two researchers worked independently to code survey data and then exchanged data to examine and discuss any category differences. After reaching agreement on categories for all data, the researchers searched for explanatory patterns to connect each coded category. Six issues summarized the results.

The first issue concerned teacher participation in decision making. The form of participation influenced how teachers viewed the participation. Unless it was obvious to teachers that the SMT used teacher input, teachers did not perceive their involvement as active participation in decision making. The researchers observed that how SMT involved teachers, then gathered and subsequently used teacher input, was significant regarding 
teachers' perceptions of the SMT and ownership of SMT decisions (Turnbull \& Mee, 2003).

The second issue addressed problems with information sharing. Staff members did not receive reports from the SMT in a timely manner. Lack of additional staff to handle communication created a time lapse in disseminating SMT information. Turnbull and Mee (2003) noted that systematic and user-friendly communication procedures were essential for participatory decision making.

The third issue concerned a "we/they" mentality between SMT members and nonmembers. An attitude of mistrust existed between some SMT members and non-members due to several factors, including the political context of SMT implementation. Another possible factor included the allegation that some SMT's chose reform models based on personal preference rather than on school-wide needs. Traditions of teachers-as-peers prohibited acceptance of teacher authority personified in the SMT (Turnbull \& Mee 2003).

The fourth issue addressed a request from every participating school for more training and more effective state help. According to Turnbull and Mee (2003), teachers expressed worry that SMT members were unprepared and lacked the necessary knowledge to plan for school improvement. Lack of sufficient background knowledge could have potentially resulted in decreased decision-making authority for the SMT.

The fifth issue raised a question about the jurisdiction of the SMT. Turnbull and Mee (2003) indicated teacher uncertainty about whether the principal or the SMT possessed authority over core school issues. The authors interpreted the data to mean that teachers might not have completely understood the extent of the authority of the SMT. 
The sixth issue addressed a lack of tolerance for anyone who disagreed with SMT decisions. SMT members considered non-member teachers who questioned SMT resolutions uncooperative and disloyal. Turnbull and Mee (2003) observed that evaluators needed to help schools develop a culture of trust and mutual respect.

Turnbull and Mee (2003) concluded that these six issues were not unique to the schools involved in this study. These problems remain common for many school governing bodies. The data from this study suggested that many schools do not know how to work through these issues and need external help to name the problems and generate solutions. These six issues could provide a starting point for SMT developers in working with schools implementing the school management team concept.

Thompson, Gregg, and Niska (2004) employed mixed methods to examine whether or not teachers and the principals in six middle schools believed their schools were professional learning communities, as well as whether students learned as a result of this status. Participants included principals and teachers from three urban and three suburban schools, all from six separate school districts. Additionally, the principals from these schools acknowledged publicly their schools' implementation of Turning Points 2000 guidelines. Data collection consisted of interviews with principals, focus groups with teachers, and administration of a truncated form of the Learning Organization Practice Profile Survey to teachers. Principal interviews uncovered beliefs about leadership and learning. The 25-question teacher survey revealed characteristics of schools as learning organizations in relationship to systems thinking, personal mastery, mental models, shared vision, team learning, relationships, and data-informed decision making. Cross-section teacher focus groups offered member checks for trustworthiness. 
Thompson et al. (2004) found that all six participating middle schools viewed themselves as learning organizations. Principal interviews ascertained with specific evidence that everyone in the school was involved in learning. Teacher surveys and focus groups reiterated the systems and practices that substantiated the schools' status as learning organizations. Emphasis on leadership which encouraged job-embedded professional development determined by the teachers significantly contributed to the culture of learning for teachers and students.

Temperley (2005) used a mixed-methods study to examine how distributed leadership affected school improvement efforts at seven low performing elementary schools in New Zealand over a 4-year period. The independent variable in the quantitative portion of the study was leadership processes involved in school improvement. The interval dependent variable was student literacy achievement at each of the seven elementary schools, as measured by annual student test scores (interval). After each school's annual test data were converted to $z$-scores, a one-way analysis of variance (ANOVA) compared current achievement data to the same students' achievement data from the previous year. The purpose of the ANOVA was to determine if significant differences existed among the seven participating schools.

Qualitative methods included interviews and observations of principals, literacy leaders, and three first grade teachers in each elementary school. Temperley (2005) observed one team meeting each year at each school, which included the school literacy leader and first grade teachers. Observations focused on key leadership activities of the literacy leaders while engaged with first grade teachers during the team meeting. The researchers interviewed the principals at each school once during each year of the study. 
Interviews of literacy leaders and first grade teachers at each school were constructed to investigate the purpose of the materials and activities observed during the team meetings. Frequency and types of observed materials and activities were tabulated. Conversations at team meetings were tape-recorded and then double coded, once by the researcher, and a second time by an independent party.

Data analysis determined that after the second year of the study, five out of the seven schools posted student achievement scores significantly higher than the other two schools. When all schools followed the same meeting and data analysis protocols in the third year of the study, there was no significant difference in student achievement scores. Analysis of leadership behaviors in team meetings revealed common themes. School vision was embedded in all meeting conversations and in underlying assumptions. Successful leaders articulated confidence in students' ability to learn. Discussion centered upon specific data analysis, individual student learning needs, intentional instruction, and instructional modeling. Literacy leaders acted as boundary-spanners between the principal and teacher-team members. Filling this role, literacy leaders effectively created coherence between the principal's beliefs and activities and those of the teachers. Literacy leaders assisted teacher-team members both in the process of analyzing student achievement data and in understanding the data's meaning (Temperley, 2005).

Distributed leadership led to shifts in power and relationships. Influence between literacy leaders and team-teachers was reciprocal. Materials, structures, and symbols, defined as artifacts, both enabled and inhibited effective discussion and decision making. Temperley (2005) concluded that artifacts were more successful when they matched both the role of the user and the task to be accomplished. Change in activities led by literacy 
leaders during team meetings facilitated change in teacher-team member vision about instruction that would improve student achievement. Temperley's study suggested when teacher leaders in different schools used similar, effective data analysis strategies, teachers' understanding of students' learning needs improved.

Grubb and Flessa (2006) examined schools which developed context-specific and non-traditional alternatives to the traditional policy of one principal per school. The researchers recruited participants through notices on websites, through personal contact with schools with which they were familiar, and through review of practitioner and research literature. Nine schools in California and one school in Massachusetts participated in the study. Each of the schools met the nontraditional principalship selection criteria. Models included schools with two co-principals, schools with rotating principals, and schools with no principal where teachers shared administrative and leadership tasks.

Data collection consisted of semi-structured interviews and observations over a period of 4 days at each school. The researchers adapted the interview protocols from the Distributed Leadership Study at Northwestern University, developing one interview procedure for school principals and district supervisors and a separate, parallel interview procedure for teachers. Parent focus groups also participated in interviews. The first segment of each interview sought information about the specific leadership format used in the school. The second segment asked for general information about the school. Other questions addressed the cost incurred, the impact on instruction, as well as student, parent, and faculty response to the change of leadership model. All interviews were taped and transcribed. Grubb and Flessa (2006) developed observation procedures for use 
during faculty, grade-level, and teacher team meetings. The researchers elected to use a selective-verbatim note-taking strategy during observations to log school personnel interactions and the researchers' impressions of tension or agreement. Data analysis included review of interview transcripts, site visits, artifacts, and observational field notes. The research team developed a profile for each school and used these profiles as the basis for identifying initial, emergent themes. The lead collaborators elaborated on the work of the research team and organized findings around specific themes. These themes include (a) the beginnings of the alternative administrative plan in each school; (b) the nature of distributed leadership in each school; (c) the benefits and costs of alternative leadership; (d) the role of district leadership in non-traditional school leadership success; and (e) conditions necessary to make alternative leadership models work.

Most of the schools in the study adopted an alternative leadership model because the school became too large and the workload unmanageable for one principal to handle. The distribution of decision-making, interchangeability, and specialization according to interest and expertise characterized distributed leadership in these schools. Dual principals and teacher-led schools allowed for (a) shared decision making, (b) the opportunity for principals, working together to share information, to cover the wide variety of required meetings, and (c) the opportunity for principals to specialize in elements of school, i.e., instructional improvement or support services, based on professional and personal interest and expertise. Distributed leadership offered more opportunities for teacher leadership, from teacher-led committees to responsibility for school operations. The consistent benefits reported from non-traditional leadership models included sharing the burden of decision-making with others, and increased 
accessibility of students, parents and school personnel to school leaders. Grubb and Flessa (2006) noted that these new approaches to the principalship made schools more effective, but did not resolve the problem of overwork. When school leaders were active and innovative, responsibilities expanded to fill the time and resources available.

Financial costs noted in the study varied from school to school. Potential benefits, such as increased instructional effectiveness, appeared to outweigh costs. Districts received low marks for bureaucratic interference, lack of communication, and reluctance to support fully implementation of alternative leadership models. The researchers observed that districts demonstrated no real efforts to support schools where teachers and principals initiated different leadership structures.

Grubb and Flessa (2006) identified reasons why alternative school leadership models mattered. Alternative models offered more attention to instructional practices, support services; and greater availability to students. Alternative approaches could resolve the work overload experienced by most principals and promote understanding of leadership work. Specialization and interchangeability demonstrated the possibilities of distributing responsibility between leaders and teachers. Grubb and Flessa called for researchers to consider a variety of alternative leadership practices instead of assuming that the solo-principal or hero-principal is the only workable model.

Muijs and Harris (2006) accepted a commission from the General Teaching Council for England in conjunction with the National Union of Teachers to conduct a study regarding the extent to which teacher leadership was relevant for education in the United Kingdom. Teacher leadership referred to the capacity for teachers to exercise leadership for teaching and learning both within and beyond the classroom. This study 
addressed three aims: (a) to identify different models and approaches to teacher leadership as practiced in the United Kingdom, (b) to investigate how teacher leadership might best be facilitated and developed, and (c) to determine potential relationships between teacher leadership as a form of professional collaboration and school improvement. The operational definition of teacher leadership for the purpose of this study meant facilitation of purposeful collaboration and cooperation between teachers for instructional purposes.

The researchers used a case study design. Participant selection occurred through purposive sampling. Local Education Authority (LEA) advisers and national groups recommended schools based on evidence of teacher leadership in the school that appeared to contribute to school improvement. After advisers generated an initial list of school participants, Muijs and Harris (2006) asked additional questions to shorten the list to a more reasonable number. The selection of ten schools, five primary and five secondary, resulted from the process. Four schools represented disadvantaged inner city areas. Four schools represented middle class, suburban areas, and two represented rural populations. A variety of school types and demographics in the South and Midlands of England allowed examination of teacher leadership within different contexts. Data collection consisted of semi-structured interviews with a cross section of school staff, including teacher leaders, classroom teachers, middle managers, school governors, and head teachers in each of the ten schools. School development plans and reports to the government agency responsible for school inspection provided documents. A thematic analysis framework permitted identification of emerging themes. The constant comparative method, involving anticipation, immersion, validation, interpretation, and 
analysis, guided scrutiny of all data. While pre-determined codes provided a set framework, ongoing generation of new themes and codes occurred during each round of analysis after initial coding. The researchers used a software program to code and analyze data.

Findings of the Muijs and Harris (2006) study supported five dimensions of teacher leadership in the existing literature. Teachers identified as teacher leaders shared decision making, engaged in collaboration to improve teaching and learning, actively participated in school improvement processes, participated in professional learning individually and with colleagues, and acted as advocates to affect change. Teachers in the study acknowledged participation in these shared leadership activities, even when they did not refer to themselves as teacher leaders. Teacher leader behaviors in most of the participating schools consisted of informal groups of teachers gathering to work for specific purposes. Some evidence existed about externally driven or funded projects that facilitated teachers meeting with one another both within and across schools. Schools which did not have external support expressed difficulty with providing teachers the time to meet for collaboration.

The majority of respondents in the study viewed teacher leadership as a key contributing factor to school improvement because it allowed teachers to share creatively in leadership work and responsibility, ultimately improving instructional performance. School leaders intentionally created conditions that supported teacher involvement in decision making for school-wide development. Teachers indicated that they were more likely to remain in schools where teacher collaboration with colleagues and leadership opportunities existed. Evidence from this study indicated that several initiatives 
undertaken by teachers directly contributed to school improvement. Muijs and Harris (2006) concluded that specific conditions appeared to support the emergence and maintenance of teacher leadership in schools. These conditions included a collaborative culture, effective organizational structures, strong leadership, commitment to action inquiry and data richness, innovative forms of professional development, coordinated school improvement initiatives, high levels of teacher participation and involvement, collective creativity, shared professional practice, and recognition and reward.

Muijs and Harris (2006) also noted a wide variety of barriers to the development of teacher leadership. External educational contexts, especially accountability demands and top-down government-mandated programs, placed significant weight on teachers and administrators, making distributed leadership more difficult and stifling teacher leadership initiatives. The lack of time teachers had to participate in activities outside of classroom teaching and administration proved to be an obstacle to teacher leadership development. While some teachers were willing and able to assume additional responsibilities, teachers also wanted to have a life outside of school. Teachers in some of the schools expressed a lack of confidence in their abilities to perform leadership tasks, while others identified general teacher apathy and unwillingness to assume new responsibilities as barriers to teacher leadership development. School administrators who communicated poorly or who did not want to share power or responsibility with teachers proved to be hindrances to teacher leader development. Successful school administrators in the study took active approaches, intentionally designing school improvement teams and encouraging teachers to play dynamic roles.

Muijs and Harris (2006) cautioned against over-stating effects of teacher 
leadership on school and student outcomes, maintaining that not enough is currently known about how teacher leadership influences schools. The researchers stated that professional learning communities appear to create and support sustainable improvement because they build teachers' skills along with the capacity to keep growing. Because more studies point toward implementation of professional learning communities and teacher change, future studies should address the relationship between teacher leadership and professional learning communities, with specific focus on their effects on schools and students.

Findings from the studies in this section presented descriptions of the purpose of distributed leadership (Camburn et al., 2003; Datnow \& Castellano, 2001; Grubb \& Flessa, 2006; Muijs \& Harris, 2006: Temperley, 2005; Thompson et al., 2004) how it works, what it looks like (Chrispeels \& Martin, 2002; Harris 2002; Heller \& Firestone, 1995), as well as barriers that could prevent it from being successfully implemented (Turnbull \& Mee, 2003; Whitaker, 1997). As a model of distributed leadership, teacher leaders provided leadership for change through participation in shared redundant tasks, allowing teachers to share creatively in the work and responsibility of leadership with the intention of improving teacher instructional performance (Chrispeels \& Martin; Heller \& Firestone; Muijs \& Harris). Distributed leadership experienced through various schoolreform models led to teacher acquisition of expert power and greater influence on other teachers' practices as well as school decisions. The hope and intent of this intentional influence was to improve teaching and student learning within the school (Camburn et al.; Chrispeels \& Martin). Findings reiterated the critical influence of the leadership style of the principal in the implementation of distributed leadership and its subsequent success 
or failure to impact instructional practice (Datnow \& Castellano; Grubb \& Flessa; Harris; Muijs \& Harris; Turnbull \& Mee; Temperley; Whitaker). Successful execution of a distributed leadership model led principals to spend more time focusing on instruction (Datnow \& Castellano) and developing instructional capacity within the school (Camburn et al.; Chrispeels \& Martin; Grubb \& Flessa; Muijs \& Harris).

Ironically, the eight qualitative studies, two mixed methods studies, and one quantitative study reviewed in this section did not include any hard data regarding the desired outcome of distributed leadership, namely improved student achievement. While the studies described how distributed leadership through teacher leadership improved both instructional practice and student learning, no data confirmed a correlation or causal connection. The research focused more on teacher and principal leadership behaviors than significant student learning outcomes. Muijs and Harris (2006) recognized the need for additional research and issued a call for more study about the relationship between teacher leadership and professional learning communities with specific focus on how these two distributed leadership models affect schools and students.

\section{Teacher Leadership and Student Achievement}

The direct link between teacher leadership and improved student achievement is far from clear (Harris, 2005). Katzenmeyer and Moller (2001) claimed that students perform better in schools where teacher leadership thrives. Barth (2001) maintained that teacher leadership allows students to observe and experience democratic leadership in schools, and thus, become the beneficiaries of better decisions about their life in school. Barth (2001) further asserted that only when teachers learn will their students learn. This section addresses research which seeks to understand the connection between teacher 
leadership and student achievement.

Crowther and Olsen (1997) considered the effect of teacher leadership on student achievement in schools with large numbers of at-risk students. The researchers conducted a qualitative study of 15 teachers and paraprofessionals identified as effective in leading change and improvement in socio-economically disadvantaged schools in Australia where they worked. Four criteria developed by the researchers and a representative group of administrators determined the fifteen participants in the study. The criteria included (a) concrete evidence of a significant contribution to a social justice in the school; (b) highly esteemed in the community, especially among socio-economically disadvantaged individuals or groups; (c) recognized by colleagues as very influential in school decision-making processes; and (d) accorded a high level of school-based responsibility by colleagues and the school administration. The purpose of the study was to determine what characteristics distinguished the work of these teachers who had achieved success in socio-economically disadvantaged schools.

Data collection occurred through participant self-recording of critical incident strategies, interviews, and observations over a 6-week period. Critical incident strategies referred to participants' written descriptions of their perceptions and responses to specific situations involving socio-economic disadvantage which occurred in their workplace. The 15 participants recorded a total of 43 critical incidents over a 4 -week period. Crowther and Olsen (1997) conducted on-site, 1-hour interviews with all participants to clarify their written responses and to discover the processes that resulted in their high levels of influence. The researchers also facilitated two 3 -hour focus group meetings that included all 15 participants. One session occurred before participants recorded experiences of 
socioeconomic disadvantage and the other after on-site interviews had been completed. During these focus group meetings, the 15 participants reflected upon their work and discussed the types of leadership activities and styles that proved successful for each of them. Data analysis involved three processes. First, a holistic profile was developed for each participant. Second, the researchers categorized data to generate theories of disadvantage, educational strategies, and leadership. Third, researchers shared findings with study participants to determine if the outcomes reflected real life.

Results of the data analysis indicated four different types of disadvantage that influenced the schools and students of the study participants. These included (a) deficits in social competence, (b) material means, (c) relationships with authority, and (d) attitudes toward school. Essential leadership characteristics that afforded the participants success in dealing with these types of disadvantage reflected transformational, educative, and strategic approaches to leadership. Clear views about marginalized individuals and groups, contagious enthusiasm, the ability to inspire others, and persistent optimism were related to transformational leadership characteristics. Continuous involvement in solving problems not of their making, collaboration to raise awareness of issues, and advocacy for the powerless signaled educative leadership characteristics. Organization of tasks with relative ease and promotion of collaboration described strategic leadership skills. Crowther and Olsen (1997) concluded from the teacher participants that their roles as teachers were both inextricably linked to, and absorbed into, their roles as influential leaders within their schools and communities. While the researchers' qualitative approach did not yield measurable data about the impact of teacher leadership on student achievement, Crowther and Olsen added to the 
literature about the nature of teacher leader work in relationship to student achievement.

Searching for quantifiable evidence, Leithwood and Jantzi (1999) explored the effects of principal and teacher leadership on student engagement in school, as well as school conditions that impact principal and teacher leader influence. The study also examined the variation in school conditions explained by teacher and principal leadership and the variation in student engagement explained by school conditions. School conditions acted as a mediating variable, one that intervenes between the independent and dependent variable and transmits the effect of the independent variable to the dependent variable. School conditions included (a) purposes and goals, (b) planning, (c) organizational culture, (d) structure and organization, and (e) information collection and decision making. Purposes and goals referred to appropriate school-wide improvement plans owned by all school members and evident in school-wide instructional and professional practice. Planning meant understanding the significance of the outcomes of goal achievement. Organizational culture referred to shared beliefs, values, accepted practices, and assumptions which shaped the decisions, policy implementation, and how people interpreted both of these behaviors. Structure and organization meant the relationships between and among individuals and groups in the school, and between the school and its external publics. Information collection and decision making referred to the kinds of information collected and used to make decisions, how school personnel used available information, who was involved in decision making, and how decisions evolved.

The study also examined the variation in principal leadership and student engagement explained by family educational culture. Leithwood and Jantzi (1999) 
selected family educational culture instead of socio-economic status because the literature indicated that socio-economic status did not reflect the innumerable family interactions which impact student perceptions and attitudes about education. Family educational culture served as a moderating variable, that is, one which influences the relationship between two other variables and produces an interaction effect between itself and other variables. Family educational culture referred to family work habits, academic guidance and support, parents' academic and occupational aspirations, home health and nutritional conditions, and a physical setting conducive to academic work.

The interval level dependent variable of interest for this study was student engagement measured through survey data. The operational definition of student engagement incorporated both behavioral and affective elements. The behavioral element included the level of student participation in school activities inside and outside of class time. The affective element was the degree to which students felt they belonged to the school. The researchers based this operational definition on previous research which concluded that students who feel that they belong to the school will value school-defined success, i.e., academic success. The interval level independent variable was principal and teacher leadership measured through survey data. Teacher leadership meant the level of influence that teacher leaders exerted as individuals or in groups in formal or informal leadership functions. Principal leadership referred to practices by the principal that impacted teaching and learning in the school.

Data collection consisted of the administration of two surveys in one eastern Canadian school district representing median income families. Leithwood and Jantzi (1999) received complete survey data from 110 out of 123 schools, yielding a response 
rate of $89 \%$. The Organizational Conditions and School Leadership Survey gathered data from 1,762 teachers with regard to school conditions and leadership. With 142 items related to school conditions and the perceptions of teacher leader and principal influence in the school, this survey employed a 5-point Likert scale rating system with anchors ranging from 1 = strongly disagree to 5 = strongly agree and a sixth option of not applicable. Another part of the survey utilized a 4-point Likert scale with anchors ranging from $1=$ minimal influence to $4=$ very strong influence to measure the level of teacher leader influence on school activities. A similar section of the survey assessed the influence of the school administrators. The 52-item Student Engagement and Family Culture survey assembled information from 9,941 students regarding their level of engagement with school and their family's educational culture. A 5-point Likert scale with anchors ranging from $1=$ strongly disagree to $5=$ strongly agree with a not applicable option gauged student experience.

Leithwood and Jantzi (1999) conducted an extensive research review about school conditions that affect student engagement. The researchers convened a panel of teacher and administrator volunteers who assessed, clarified, and revised survey items. The researchers then performed a pilot test in one school district before making further revisions and publishing the surveys. A final step in establishing content validity consisted of two additional reviews by two different sets of teachers, consultants, and administrators who made additional revisions. After both the initial pilot test and their 1998 study, Leithwood and Jantzi calculated Cronbach's alpha for all scales in both surveys to establish internal reliability. Scale reliabilities ranged from .70 to .97 in the pilot test and .70 to .97 in the 1998 study. 
Due to the large number of items to be measured, Leithwood and Jantzi (1999) elected to develop two forms of the Organizational Conditions and School Leadership Survey. Schools with fewer than 10 teachers completed both forms of the survey at two different times due to length of time needed to respond to all items. In all other schools in the district, each teacher randomly received either Form A or Form B. The number of respondents for Form A was 888 and 874 for form B, a total response rate of $71 \%$. All students in one class of the three highest grades of each school completed the Student Engagement and Family Educational Culture Survey. Response rate was $100 \%$ of students attending class on the day the survey was administered by the principal, a representative $22 \%$ of the district's student population. The researchers combined individual responses by school and computed means, standard deviations, and Cronbach's alpha for scales measuring each variable. Analysis of school conditions occurred through extraction of principal factors with varimax rotation to determine the maximum variance provided by each factor (Vogt, 2005). Analysis of covariance tested for validity of causal inferences for pairs of variables, while controlling for the effects of other variables. A series of regression analyses followed to estimate how principal and teacher leadership impacted school conditions listed in the study, as well as how these school conditions affected student engagement.

Study results indicated that the effects of teacher leadership on student engagement were not significant. Principal leadership, although not strong, possessed some statistical influence on this student variable. Leithwood and Jantzi (1999) suggested that student engagement, as defined in their study, unlike student achievement, typically was not tied to classroom instruction. 
Leithwood and Jantzi (2000) chose to repeat their 1999 study regarding the relative effects of principal and teacher leadership on student engagement (Leithwood \& Jantzi, 1999). The researchers asserted that replication of earlier research built a strong knowledge base, and therefore was valuable. The interval level dependent variable was student engagement, which included both behavioral and affective elements. The behavioral element meant the extent of students' involvement in all school activities, academic and extracurricular. The affective element referred to the degree to which students felt part of the school organization. Interval level independent variables consisted of principal and teacher leadership, with moderator variables of school and classroom conditions, as well as family educational culture. Family educational culture referred to family work habits, academic guidance and support, parents' academic and occupational aspirations, home health and nutritional conditions, and a physical setting conducive to academic work. Leadership referred to the process of influencing others and being perceived as a leader. Principal leadership indicated behaviors and procedures that directly affected teaching and learning. Teacher leadership referred to the degree of influence that informal or formal teacher leaders, individually or collectively, had on the school. School conditions addressed purposes and goals, school planning procedures, school organizational culture, and, finally, school structure and organization. Classroom conditions referred to instructional services by teachers and policies and procedures that guided decision making for school initiatives.

The participant pool consisted of 2,424 teachers and 7,251 students from 98 elementary schools in a large school district in central Canada. This school district possessed similar demographic characteristics to the school district used in the original 
study. Data collection occurred through the use of two surveys. The first survey measured teachers' perceptions on school and classroom conditions and on leadership roles within the school. The original survey consisted of 228 items rating eight different sets of information on a 5-point Likert-type scale, using anchors of $1=$ strongly disagree to 5 strongly agree, with an option for not applicable. Due to the length of the survey, researchers developed two separate forms and randomly distributed them to participants. Both forms gathered information about some school and classroom conditions and all leadership roles. The return of 1,818 useable surveys from 94 schools resulted in a $75 \%$ teacher response rate (Leithwood \& Jantzi, 2000). The second survey measured students' views on their level of engagement with school and their families' educational cultures. Students in the highest grade at each elementary school had an opportunity to respond to the survey administered by the school principal. Out of 7,251 possible participants, 6,490 students contributed data, a response rate of $90 \%$. Prior use of both surveys revealed high degrees of internal reliability. Data analysis consisted of principal components analysis with varimax rotation. LISREL assessed the direct and indirect effects of principal and teacher leadership on student engagement through an analysis of covariance structure approach to path analysis. In addition, teacher and student survey results were linked to their specific schools.

Results indicated that principal leadership $(r=0.73)$ had a more significant relationship with school conditions than did teacher leadership $(r=0.48)$. Neither principal leadership nor teacher leadership showed a significant relationship with family educational culture or student participation, a component of student engagement. Principal leadership was significantly related with student identification $(r=0.25)$, the 
second element of student engagement, while teacher leadership was not. Family educational culture had a significant relationship with both elements of student engagement, participation $(r=0.70)$, and identification $(r=0.71)$. Principal leadership explained $7 \%$ of the variation in teacher leadership. Leithwood and Jantzi (2000) concluded from this study, as from the study in 1999, that neither principal nor teacher leadership accounted for a significant percentage of the variance in student participation. Principal leadership explained a significant percentage of variance only in student identification with the school. In spite of the advocacy for teacher leadership as part of school reform, there remains no quantitative evidence to support its positive impact on student engagement.

Similarly, Wenglinsky (2002) used quantitative methods to study directly the link between student outcomes and teacher quality, although this investigation was for academic achievement. Teacher quality referred to classroom practices, professional development activities, and background characteristics. The study was important because quantitative research previously reported that effects of student background on student achievement overshadowed the impact of classroom instruction. Based on the literature, the author hypothesized that teacher classroom practices had the greatest effect on student academic performance, followed by professional development and background characteristics, respectively. An additional hypothesis claimed that all three criteria of teacher quality were as strongly related to student academic performance as were student background characteristics. The independent variables included teacher background, professional development, and classroom practices. The dependent variable was student academic achievement. 
The sample included math data from 7,146 eighth graders who took the 1996 National Assessment of Educational Progress (NAEP) mathematics assessment. Test scores measured student achievement. A student questionnaire gathered data about student background. The NAEP gathered data about mathematics teachers' education levels, recent professional development activities, and typical classroom strategies (teacher quality) through questionnaires. Wenglinsky (2002) used factor analysis and path analysis models to analyze the data. The factor analysis model related observed variables to a latent variable. Latent variable refers to an underlying characteristic that cannot be observed or measured directly, but is presumed to exist in order to explain factors that can be observed (Vogt, 2005). The path analysis model connected the latent variables to one another in a set of hypothesized relationships designated by the researcher.

Path analysis results of the study by Wenglinsky (2002) indicated that the teacher's level of education was not significant to student achievement $(\beta=.09)$. The effect of student socioeconomic status $(\beta=.76, p<.05)$ was largest. The path analysis for professional development indicated that a significant relationship existed between student achievement and teachers' professional development experiences regarding special student populations $(\beta=.21, p<.05)$ and training in higher order thinking skills $(\beta=.12$, $p<.05)$. Student socioeconomic status yielded the largest effect $(\beta=.83, p<.05)$ in the professional development path model. The path analysis for classroom practice noted significant relationships between student achievement and use of hands-on learning activities $(\beta=.25, p<.05)$, solving unique problems $(\beta=.13, p<.05)$, and refraining from use of authentic assessments $(\beta=-.18, p<.05)$. Again, student socioeconomic 
status produced the highest effect size $(\beta=.74, p<.05)$. The researcher computed total effect size for each path analysis by summing the direct and indirect effects of each teacher factor in each separate path analysis. The total effect size of teacher background on student achievement was .09 , while the total effect size of teacher professional development experience on student achievement was .33. Effect sizes for classroom practices equaled .56.

Wenglinsky (2002) concluded that professional development in higher order thinking skills and in diversity, as well as hands-on learning experiences and the use of higher order thinking skills in instructional practice were positively associated with student achievement. The outcomes supported both of the researchers' hypotheses. Wenglinsky noted the strong influence of professional development on teachers' classroom practices. Study limitations included use of cross-sectional data, and the fact that the study covered data from one grade level in one subject. The study did not assess links between teacher quality and the relationship between student achievement and student socioeconomic status. Single indicator constructs in teacher and student NAEP surveys provided insufficient information about socioeconomic status, teacher professional development, and classroom practices. A significant flaw in the study stems from the unexplained differences between the original operational definitions of the NAEP questionnaire data and the post hoc meaning assigned to the data by Wenglinsky. Building upon the literature, Silins and Mulford (2004) inspected the impact of leadership practices and processes on organizational learning and student outcomes, with emphasis on the relationship of teacher leadership to two sets of variables connected to school improvement initiatives: school context variables and internal school variables. 
The interval level independent variable of the study consisted of leadership practices. School context variables consisted of socioeconomic levels and school size. Procurement of school context information occurred through analysis of school demographic data. Internal school variables included (a) resource, described as the availability of resources to teachers; (b) leader, referring to the principal's implementation of transformational leadership constructs; (c) staff valued, defined as the level at which staff members feel welcome and accepted; (d) leadership satisfaction, explained as the degree of satisfaction with all sources of leadership within the school; (e) community focus, interpreted as the scope of school administrators' working relationship with the larger community; (f) teacher leadership, meaning the extent to which teachers individually or collectively provide leadership to the school, (g) organizational learning, described as the level at which the school operates as a learning organization according to four previously established criteria; and (h) teachers' work, defined as students' views of teachers' instructional practice, class organization, and expectations of student achievement. The dependent variables for the study, measured on the interval scale, included student outcomes defined as participation and engagement with school and organizational learning. Participation meant student attendance, level of extracurricular involvement, doing extra schoolwork, setting personal learning goals, and speaking freely in class. Engagement referred to students' views on how teachers interacted with them, how students interacted with fellow students, how class work was connected to real life, and how much they felt themselves a part of the school. These operational definitions are similar to those of the Leithwood and Jantzi studies $(1999,2000)$. Organizational learning meant the extent to which school leaders include staff members in leadership 
development and decision-making, where decisions are data-driven, where a common vision is articulated, and where communication flows between all stakeholders. Data for this study came from surveys of teacher and student participants in a larger 5-year study known as the Leadership for Organizational Learning and Student Outcomes Project (LOISO). Survey data gathered from 2,503 teachers and their principals from 96 secondary schools led to identification of four characteristics of high schools managed as learning organizations. Further analysis of teacher survey responses from these surveys yielded information about transformational organizational practices. A second survey of 3,500 sophomores from the original 96 secondary schools reported data regarding family educational environment; students' views of teacher classroom work; and specific student outcomes, i.e., attendance, self-concept, participation, and engagement in school. Measures for both surveys utilized anchors which ranged from $1=$ strongly disagree to $5=$ strongly agree. Data from both of these surveys served as the basis for Silins and Mulford's (2004) study. A path model used latent variable partial least squares path analysis procedure. Each variable position relied upon its predicted influence on all succeeding variables in the model. Analysis occurred in two steps. The first step pertained to the outer path model. Factors in the outer model that retained at least twice their standard error and were equal to or greater than 0.40 were kept. The second step, dealing with the inner model, followed the same process. Deletion of the path occurred if the path coefficient was less than twice its standard error, or less than 0.10 .

Results showed five variables as direct predictors of organizational learning: (a) teacher leadership ( $\beta=0.26$ ); (b) staff valued ( $\beta=0.26$ ); (c) leadership satisfaction $(\beta=0.22)$; (d) leader $(\beta=0.19)$; and (e) resource $(\beta=0.17)$. For schools functioning as 
learning organizations, teacher leadership implied that all staff members worked to influence school activities, with some teachers serving as informal leaders. Resources referred to a positive perception of the availability of resources to carry out instructional tasks. An additional three variables were path coefficients of teacher leadership: (a) staff valued $(\beta=0.37)$, (b) leadership satisfaction $(\beta=0.36)$, and (c) socioeconomic status $(\beta=-0.21)$. Staff valued referred to all school employees feeling valued by school leadership. Leadership satisfaction indicated that school personnel were satisfied with all sources of leadership within the school. Silins and Mulford (2004) conjectured that the negative path value of socioeconomic status indicated a higher level of teacher leadership in lower level socioeconomic schools. Teacher leadership played a significant role in the organizational leadership of surveyed schools. Teachers' work in the classroom was the strongest path coefficient predictor of student participation $(\beta=0.51)$ and student engagement $(\beta=0.75)$.

Leithwood and Jantzi (2006) built upon previous research which found that school leadership effects were the same regardless of leadership style. Leithwood and Jantzi chose to test the effects of a school-specific model of transformational leadership on teachers, their classroom practices and gains in student achievement. This study was part of a larger evaluation of England's National Literacy Strategy (NLS) and National Numeracy Strategies (NNS) authorized by the British government and conducted over 4 years. The independent variable was transformational leadership and the dependent variables included teacher classroom practices and student achievement. Motivation, capacity, and work setting served as mediating variables (see Figure 5). 


\section{Leader Practices}

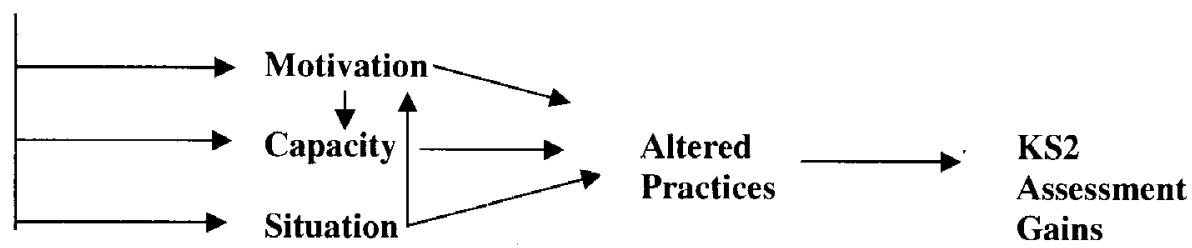

Figure 5. Theoretical framework explaining leaders' effects on teachers and their practices.

A mediating variable is one that intervenes between the independent and the dependent variable and transmits the effects of the independent variable to the dependent variable. These three variables reflected the outcomes regarding employee performance in the literature on organizational and industrial psychology.

In the study by Leithwood and Jantzi (2006), transformational leadership referred to three categories of leadership practices: setting directions, developing people, and redesigning the organization. Motivation meant the characteristics of someone who looks ahead to the future and decides if change is necessary in order to reach personal goals or develop personal capabilities. Emotion stimulated motivation and self-belief. Capacity referred to the ability to complete a task accurately. In the school setting, capacity meant more than the sum of each person's ability to do a job. Work setting referred to teachers' common practices related to reform, as well as the physical and social structures that supported these practices. Work setting also meant the effectiveness of the staff as a whole. Teachers' classroom practices consisted of implementation of changes in the teaching of literacy and mathematics and increased time spent in student learning. Student achievement referred to measures of student performance on the NLS and the NMS. The sample for the study consisted of randomly selected primary schools, including independent schools, from England's National Foundation for Educational Research database. One sample of 500 schools provided data about the NLS. Another 
500-school sample gave information about the NNS. The research instrument consisted of two forms of a teacher survey which used a 5-point Likert scale with anchors ranging from 1 = strongly disagree to 5 = strongly agree. These two survey forms measured motivation, capacity, and work setting. After field testing and other improvement processes not specified by the researchers, headteachers in the randomly selected schools received the surveys and distributed them to teaching staff. Leithwood and Jantzi gained access to each school's results for the NLS and NNS from England's Qualifications and Curriculum Authority (QCA).

The Statistical Package for the Social Sciences (SPSS) facilitated calculation of descriptive data, reliability, and correlation coefficients. Independent sample t-tests compared means of literacy and numeracy respondents. Paired sample t-tests compared means of specific strategies in each test, i.e., reading and writing in literacy. Path analysis, a form of structural equation modeling, used LISREL to determine the direct and indirect effects of leadership on the mediating variables (motivation, capacity and work setting) and teacher classroom practice. The school response rate for the NLS was $64 \%(N=340)$ and $63 \%(N=315)$ for the NNS. The teacher response rate was $20.4 \%$ $(N=1110)$ for literacy and $20.3 \%(N=1,190)$ for numeracy. The researchers indicated two methodological limitations of the study. The first was the low teacher response rate. While teacher response rate was low, Leithwood and Janzti (2006) believed that the size of the sample was significantly larger than most educational leadership research samples. The sample size was similar to the total population of primary teachers in England in age, ratio of male to female teachers, and years of teaching experience. The second noted limitation was the measure used for student achievement gains. The researchers noted 
that gain scores over only a 2-year period may be unstable and agreed that tracking gains over 3 or more years would yield more valid data. The researchers tracked numeracy data over 1 year and literacy data over 2 years in this study, and chose to run all data a second time using mean annual achievement scores instead of gain scores. There were no significant differences in the results.

Findings from this study revealed that transformational leadership had strong direct effects on teachers' work settings and motivation, but less significant effects on teachers' capacities. Transformational leadership had a moderately significant effect on teachers' classroom practices, explaining $25 \%$ to $35 \%$ of the variance. The most significant finding was that none of the variance in student achievement gains could be explained by transformational leadership practices. The researchers suggested that there is a gap between a mere change in teachers' classroom practice and classroom practices that lead to higher levels of student achievement. Leithwood and Jantzi (2006) note two implications for further study. The first implication suggests an examination of which sources would best deliver specific leadership practices that would most significantly impact student achievement. The researchers remark that distributed leadership has garnered support in school districts in spite of limited empirical evidence about its results. A second implication is related to large scale studies of leadership effects. Data collection in this study included samples of leaders who ranged from highly successful to mediocre. The authors indicated the pressing need for large-scale studies of strong leadership and the nature and size of its effects on students and schools.

The studies in this section were primarily quantitative in nature, reversing the trend noted in previous sections. While the findings added to the literature about the 
impact of principal leadership and teacher leadership on student engagement or student outcomes (Leithwood \& Jantzi, 1999, 2000; Silins \& Mulford, 2004;), neither student engagement nor student outcomes meant the same thing as student achievement. Each researcher in this section made this distinction clear through operational definitions. The assumption was that student engagement would lead to student achievement (Leithwood \& Jantzi; Silins \& Mulford). The resulting data suggested that while principal leadership had some influence on student engagement, teacher leadership had no influence on student engagement. Thus, even if student engagement connoted student achievement, the results were far from what might have been expected. To clarify, however, the survey data from Leithwood and Jantzi as well as Silins and Mulford measured teacher and student perceptions of their schools, instructional services, principal and teacher leadership, rather than hard data that measured student achievement. In this section, only Wenglinsky (2002) and Leithwood and Jantzi (2006) used hard student achievement data. Wenglinsky used data from the math portion of the 1996 National Assessment of Educational Progress (NAEP). However, Wenglinsky's study was flawed because of the unexplained differences between the operational definitions of the NAEP questionnaire data and the post hoc meanings which he later applied to that data. Leithwood and Jantzi recognized that the measure used to demonstrate student achievement gains was unstable because it did not span enough time. Even so, their 2006 study suggested that student achievement gains could not be explained by leadership practices. Thus, Leithwood and Jantzi called for studies that would indicate leadership sources which could successfully impact student achievement. 


\section{Theoretical Framework}

Lave and Wenger (1991) developed the theory of situated learning which explained learning as a social practice involving co-participants in learning by doing. Situated referred to the premise that abstract understandings or beliefs become meaningful during specific events in time and place. Learning was situated in a specific context and practice. Knowing and doing were intertwined and inseparable (Brown, Collins \& Duguid, 1999). In contrast to the perception of learning as the transmission of knowledge in a largely cerebral process, Lave and Wenger viewed learning through the lens of social practice.

Social practice emphasizes the relational interdependency of agent and world, activity, meaning, cognition, learning, and knowing. It emphasizes the inherently socially negotiated character of meaning and the interested, concerned character of the thought and action of persons-in-activity... participation is always based on situated negotiation and renegotiation of meaning in the world. This implies that understanding and experience are in constant interaction (Lave \& Wenger, pp. 50, 52).

The researchers theorized that because learning was a social practice, the learning process not only involved individual engagement in specific activities, but also involved becoming a member of a community of practice, i.e., a group of people for whom specific functions, activities, and tasks derive meaning through sharing a common goal. "Learning is not merely a condition for membership, but is itself an evolving form of membership" (Lave \& Wenger, 1991, p. 53). The community of practice developed knowledge and skills specific to their work. Active engagement in that process both reproduced and transformed the community of practice. New members were assimilated with previous members through interaction and conversation related to authentic tasks to continue sustained participation and development of the community of practice. On- 
going learning-in-practice transformed the identities of individuals into a generative community capable of "producing its own future" (p. 58).

Research regarding the community of practice (Lave \& Wenger, 1991) expanded with the work of Hord (1997); Dufour and Eaker (1998); the National Staff Development Council (NSDC) with new Standards for Staff Development (2001b); the Annenberg Institute for School Reform (2004); Dufour, Eaker and Dufour (2005); and Dufour, Dufour, Eaker and Many (2006). Hord reviewed what the literature termed professional learning community and described what happened when a school initiated this structure. The results suggested that professional learning communities could increase the school's capacity to meet student learning needs. Dufour and Eaker described a professional learning community model for schools "characterized by a shared mission, vision, and values; collective inquiry; collaborative teams; an orientation toward action and a willingness to experiment; a commitment to continuous improvement; and a focus on results" (p. 45). The NSDC contended that if staff development were to improve the learning of all students, it must "organize adults into learning communities whose goals are aligned with those of the school and district" (NSDC, 2001b, p. 1). These learning communities must share a commitment to continuous improvement in teaching practices and student learning (NSDC, 2001a). The Annenberg Institute for School Reform (AISR) at Brown University encouraged the use of professional learning communities based on research findings indicating improved instructional capacity in the classroom resulted in student gains in academic achievement. Professional learning communities provided opportunities for teachers in schools to learn and think together about how to improve their instructional practice to improve student learning. Dufour et al. (2005) assembled 
articles from numerous renowned educational researchers, all of whom enthusiastically supported the concept of the professional learning community to improve the quality of teaching and learning. "There is no good reason to delay this reform. It is time for a concerted effort to push for its inclusion in state department requirements, in every preservice and leadership training course, and in every discussion among principals and teacher leaders that purports to improve teaching and learning" (p. xiv). Dufour et al. (2006) defined professional learning community as a "collaborative team whose members work interdependently to achieve a common goal linked to the purpose of learning for all" (p. 3). The researchers described the what, why, and how of professional learning communities to guide schools in developing their own.

Similarly, teacher leadership neither developed nor operated within a vacuum. Knowledge was both personal and social. What an individual knows is determined by what that person has experienced doing. Promoting learning required allowing individuals to begin with where they are and from there move into new ways of thinking and doing (Darling-Hammond, 1993). The school social context, or culture, provided opportunities for informal connection between teachers to learn and strengthen the established school norms. This same school context could be the scene of opportunities for teachers to begin where they are and then, develop specific, job-embedded knowledge and skills through intentional interaction with one another in order to generate greater student learning. Full teacher participation in such a community of practice could evoke focused commitment of time, concentrated effort, assumption of greater responsibilities within the school, willingness to take risks, and a growing sense of identity as a teacher who is a leader. Active engagement in these opportunities could transform the school into 
a generative community of teachers who are reflective students of instructional outcomes. Thus, learning by doing in real-life context as prescribed by the theory of situated learning (Lave \& Wenger, 1991) could provide a framework for understanding how teacher leaders' interactions with teachers could impact student achievement.

Lambert (1998; 2003a; 2003b; Lambert \& Harris, 2003) suggested a theoretical model of leadership capacity development which provided another perspective for viewing how teacher leaders' interactions with teachers might affect student achievement. The framework, derived from literature on school reform, includes five constructs which could be applied to the interactions between teachers and teacher leaders. First, opportunities for broad-based, skillful participation in teacher leadership activities exist within schools. This means that school organizational structures support opportunities for teachers and teacher leaders to collaborate on meaningful tasks. In addition, occasions for teacher leaders to observe, practice, and receive coaching on key collaborative skills, such as facilitating, communicating, and mediating, are available. Second, an inquiry-based use of information shapes school decisions and instructional practice. This suggests that reflection, conversation, and posing of questions in response to data are regular interactions between teachers and teacher leaders. Third, roles and responsibilities indicate more widespread involvement and collaboration among teachers. This element points toward changes in teachers' perceptions about themselves and their role in the work of the school as a result of on-going interaction with teacher leaders and colleagues. These changed perceptions can lead to changed relationships and instructional practices. Fourth, reflective practice and innovation are the norm. Conversations between teachers and teacher leaders that call for reflection about beliefs, 
assumptions, content standards, learning styles, and instructional practice become common and non-threatening. Collaborative planning, monitoring, and evaluating innovative and risk-taking ideas is encouraged through regular interaction. Finally, high student achievement is evident. This element of the framework means that students successfully perform authentic tasks, i.e., tasks that have a real-world relationship to work or the general society. Achievement data come from multiple types and forms of assessment at various points in time and is used as evidence to determine who needs specific interventions to master the content and who needs deeper learning opportunities. The goal of achievement data is to support continuous improvement for all students. According to Lambert, this continuous student improvement is the ultimate purpose of all school leadership. For the purpose of this study, it is the primary purpose of all interactions between teacher leaders and teachers and will be referred to as analysis of learning.

For Lambert (1998), student achievement (analysis of learning) was defined in broad terms and included much more than scores on high-stakes accountability tests as used in this study. Student achievement included first and foremost the ability to successfully perform real-world tasks valued by society. Lambert added to this definition the development of positive affective behaviors, i.e., involvement in school life as evidenced by regular attendance and increased graduation rates. Resilient behavior patterns, as demonstrated in student self-direction, problem solving, and social competencies, were also critical elements defining student achievement (analysis of learning). Lambert viewed student achievement as "continuous improvement over time, with improvement increasing and gaps narrowing the longer that students [were] exposed 
to school improvement factors" (1998, p. 24). In this study, the researcher will use analysis of learning for Lambert's student achievement construct.

The theoretical framework that will shape this study comes from the work of Lave and Wenger (1991) and Lambert (1998; 2003a; 2003b; Lambert \& Harris, 2003). Situated learning (Lave \& Wenger) suggests that learning is social, that learning and doing cannot be separated, and therefore that job-embedded learning provides the most meaningful opportunities for professional growth. Lambert proposes that successful schools possess (a) broad participation in leadership activity, (b) inquiry based use of data, (c) multiple role opportunities for teachers, (d) reflective practice, and (e) analysis of learning. Teacher leaders enact these premises in their daily interactions with teachers in order to bolster student achievement.

\section{Summary}

The literature on teacher leadership is abundant and reflects the progressive changes in this role since the 1990's. Qualitative studies sought to identify characteristics necessary for effective teacher leadership. Findings suggested that teacher leaders were enthusiastic about teaching and experienced job satisfaction (Beachum \& Dentith, 2004; Birky \& Ward, 2002; LeBlanc \& Shelton, 1997). Teacher leaders pursued lifelong learning opportunities, desired positive peer relationships (Birky \& Ward; LeBlanc \& Shelton) and recognition for the roles they played and their accomplishments (LeBlanc \& Shelton). Teacher leaders also sought opportunities to engage in meaningful, challenging work involving collaboration with colleagues who shared the same values (Beachum \& Dentith; Birky \&Ward). One might legitimately expect these characteristics to be common for all teachers, not reserved to those classified as teacher leaders. 
Teacher leader involvement in school-level decision making was anticipated to positively impact student achievement. Surprisingly, studies indicated that allowing teachers to exercise leadership through school-based decision-making did not result in changes in instructional practice (Griffin, 1995; Taylor \& Bogotch, 1994) or student achievement (Smylie et al., 1996; Stone et al., 1997; Taylor \& Bogotch). Additionally, the strong cultural norm of individual teacher autonomy was identified as the primary reason why teachers were hesitant to challenge the status quo through participating in decision-making that would lead to school change (Cameron, 2005; Griffin, 1995; Smylie, 1992a). Such an atmosphere of isolation and distrust of colleagues who would lead change rendered this level of teacher leadership practice ineffective for student achievement gains.

When the focus of teacher leadership research shifted to classroom teachers who taught full time, influenced teacher colleagues, and performed additional leadership duties, the results were similar. Teacher leaders saw their primary function as supporting the instructional practice of teaching colleagues in their schools, developing knowledge and insight about instruction, and influencing school-wide leadership decisions (Beattie, 2002; Cruz, 2003; Frost \& Durrant, 2002; Hatch et al., 2005; Little, 1995; Mangin, 2005; Sawyer, 2001; Silva et al., 1996; Smylie \& Denny, 1990; Spillane et al., 2003). However, when compared to other job responsibilities, teacher leaders spent less time interacting with peers about teaching practices (Smylie \& Denny) than in any other leadershiprelated activities. Although classroom teacher leaders saw their work as important for the growth of the school and their students (Cruz; Middlebrooks, 2004; Ovando, 1996; Silva et al.), they also experienced a heavier work load, less personal time, and loss of 
undivided attention to classroom instruction. Once again, the qualitative methods used in the majority of these studies described the general experiences of classroom teachers who acted as teacher leaders, but did not shed any light on the degree to which teacher leaders impacted teaching and learning.

Principals played a significant role in the success of teacher leaders (Andersen, 2004; Brown \& Anfara, 2002; Hart, 1994; Smylie \& Brownlee-Conyers, 1992). Negotiating a new working relationship between principal and teacher leader involved a transition from independent to interdependent actions focused on school improvement (Foster, 2004; Smylie \& Brownlee-Conyers). Principals who supported teacher leadership attended to the teacher leader's role as well as the faculty perception of the teacher leader role, frequently and visibly reinforcing the work of the teacher leader (Hart). Effective principals fostered leadership as a shared social influence process with all teachers sharing responsibility and influence to improve student learning. Teachers in these school settings believed teacher collaboration and continuous professional learning were of primary importance for school success (Foster). Essentially, this described how the theory of distributed leadership might look. Principals worked with teacher leaders to provide leadership for change through participation in shared redundant tasks, inviting teachers to share creatively in the work and responsibility of leadership with the intent to improve teacher instructional performance (Heller \& Firestone, 1995; Muijs \& Harris, 2006) and student learning (Camburn et al,, 2003; Chrispeels \& Martin, 2002). The critical influence of the leadership style of the principal in implementing distributed leadership was reiterated (Datnow \& Castellano, 2001; Grubb \& Flessa, 2006; Harris, 2002; Muijs \& Harris; Temperley, 2005; Turnbull \& Mee, 2003; Whitaker, 1997). 
Successful implementation of distributed leadership led principals to focus on instruction and development of instructional capacity within the school (Camburn et al.; Chrispeels \& Martin; Grubb \& Flessa; Muijs \& Harris). While these qualitative studies described how distributed leadership enacted through effective principal leadership and skillful teacher leadership could improve instructional practice and student learning, no data confirmed the connection.

The same statement was true for studies about the impact of principal and teacher leadership on student engagement or student outcomes (Leithwood \& Jantzi, 1999, 2000; Silins \& Mulford, 2004). Neither student engagement nor student outcomes were operationalized to mean student achievement, but both were expected to lead to improved student achievement. Study results suggested that principal influence had minimal significant impact on student engagement but that teacher leadership had none. Studies by Wenglinsky (2002) and Leithwood and Jantzi (2006) used student achievement data but they were either flawed or were considered unstable.

In this literature review, $64 \%$ of the studies used qualitative methods to describe the experience of teacher leadership in its variety of enactments; $20 \%$ used quantitative methods to measure the effect of principal and teacher leadership on student engagement; and $16 \%$ used mixed methods for all of these purposes. Few quantitative studies have been conducted to determine the effect of teacher leaders on student achievement. Muijs and Harris (2006) called for studies to address the relationship between teacher leadership and professional learning communities, with specific focus on their effects on schools and districts. Leithwood and Jantzi (2006) called for studies that would indicate leadership sources which could successfully impact student achievement. The purpose of 
this study is to determine the relationship between teacher leaders' interactions with teachers and student achievement as measured by the Kentucky Core Content Test (KCCT). 


\section{CHAPTER III}

\section{METHODOLOGY}

\section{Introduction}

Over the past seventeen years many studies have been conducted about teacher leadership. The research topics have been wide-ranging, encompassing teacher leaders as participants in school-based decision making to teacher leaders as instructional change agents in schools. While these studies might have been based on the assumption that teacher leadership development would lead to improvement in student achievement, few studies made this intentional connection. Researchers used primarily qualitative methods to focus on the experience of teacher leaders in the variety of roles in which they found themselves involved. Few quantitative studies were designed to investigate the relationship between teacher leadership and student achievement. The purpose of this study was to measure the relationship between teacher leader interactions with teachers and student achievement as measured by the Kentucky Core Content Test (KCCT). This chapter will include the research question, a description of the variables, the research design, the population from which the sample was taken, the survey instrument used, validity and reliability, and procedures used for data collection.

\section{Research Question}

Since the inception of education reform in Kentucky in 1990, schools 
across the state have been required to test students each spring with the KCCT. Some Kentucky schools created full-time teacher leader positions to support the work of the principal as instructional leader. Both district leaders and school principals embraced this quasi-administrative position even though no research verified a positive correlation between teacher leadership and student achievement. Other schools elected development of a school leadership team populated by representatives of each department. These leadership team members sometimes serve as the facilitators for departmental or crossdiscipline learning communities (Dufour et al., 2006) for teachers in the school. Still other schools in Kentucky may not have taken formal action to develop specific individuals as teacher leaders, but benefit, or suffer, from the results of informal teacher leadership behaviors that one or more teachers in the building regularly enact. Thus, this study holds potential value for all Kentucky schools.

In addition, well-established variables such as ethnicity and the number of students receiving free or reduced lunch have been known to correlate negatively with student test scores. Therefore, it is necessary to control for these factors to more clearly measure the relationship between teacher leadership and student achievement. This study addressed the following research question: What is the relationship between (a) interactions between teacher leaders and teachers and (b) average test scores for Kentucky schools on the Kentucky Core Content Test (KCCT) after controlling for demographic factors known to predict average school test scores.

\section{Description of Variables}

The variables named in this study were identified from the literature as well as from current practice. The independent variables were designated by the research of 
Lambert (1998). The independent variable. the KCCT, was identified because it is an established method for measuring student achievement in Kentucky.

\section{Independent Variables}

The five independent variables in this study consisted of categories of teacher leader interactions with teachers as identified by Lambert (1998). These variables included activities clustered around (a) broad-based and skillful participation in the work of leadership; (b) inquiry-based use of information to inform shared decisions and practice; (c) roles and responsibilities that reflect broad involvement and collaboration; (d) reflective practice/innovation as the norm; and (e) analysis of learning. A group of specific behaviors enacted by teacher leaders will characterize each of the independent variables. Because ethnicity and socioeconomic status have been shown to influence test scores in Kentucky, controls for these factors will be established.

\section{Dependent Variable}

The dependent variable in this study was the 2008 participant elementary school scores on the KCCT. For the purpose of this study, the Academic Index (AI) for each school was used as the KCCT score. The Academic Index is a composite of scores earned for tested content areas in Grades Three through Six. For students in third through sixth grades in elementary schools, these content areas and weight values include

(a) Reading, 19\%; (b) Mathematics, 19\%; (c) Science, 14.25\%; (d) Social Studies, 14.25\%; (e) Writing on Demand, 2.85\%; (f) Writing Portfolio, 11.4\%; (g) Arts and Humanities, 4.75\%; and (h) Practical Living/Vocational Studies, 4.75\%. All students in Grades Three through Six are assessed in Reading and Math. Students in Grade Four are also assessed in Science, Practical Living/Vocational Studies, and Writing through 
submission of a Writing Portfolio. Students in Grade Five take additional assessments in Social Studies, Arts and Humanities, and an On-Demand Writing Prompt.

\section{Research Design}

This non-experimental study examined whether specific interactions between teacher leaders and teachers impact KCCT scores. According to Pedhazur (1997), "Multiple regression analysis is eminently suited for analyzing collective and separate effects of two or more independent variables on a dependent variable" (p. 3). Because the goal of multiple regression is to "produce a model in the form of a linear equation that identifies the best weighted combination of independent variables in the study to

optimally predict the [dependent] variable" (Meyers, Gamst, \& Guarino, 2006, p. 149), this statistical method was used in this study.

According to Pedhazur (1997), controlling among variables, i.e., control of variance, refers to the "use of statistical methods to identify, isolate, or nullify variance in a dependent variable" (p. 157). This statistical control is decidedly more important when the study involves "more than one independent variable on a dependent variable, because one has to be able to sort out and control the effects of some variables while studying the effects of other variables" (p. 157). Pedhazur states that multiple regression provides such control. In this study, demographic factors known to influence KCCT scores were held constant.

\section{Multiple Regression}

Huck (2008) states that "different kinds of multiple regression exist because there are different orders in which data on the independent variables can be entered into the analysis" (p. 422). Variations of step-wise multiple regression build the model one 
variable at a time rather than all at once as does the standard multiple regression procedure. In forward step-wise regression, independent variables are added one at a time. SPSS enters each independent variable with the highest correlation to the dependent variable if the correlation is statistically significant (Meyers et al., 2006). Thus, the entire set of predictor variables may not be entered into the model, depending upon the strength of correlation between independent and dependent variables. In this study, specific demographic factors known to influence student achievement were entered first into the regression equation in order to account for any variance on the dependent variable. Any remaining variance could be attributed to the other five independent variables, which were be entered in successive steps. Forward step-wise multiple regression was appropriate to use in this study.

\section{Population and Sample}

The population for this study consisted of teachers from public elementary schools in Kentucky in the Green River Regional Educational Cooperative (GRREC) service region. Each of these public schools had participated in the spring 2008 KCCT. Elementary schools in the GRREC service region represent 32 of 174 public school districts and 124 out of 751 elementary schools in Kentucky. While these elementary schools are configured to include a variety of grade levels, most include students who are required to take the KCCT in third, fourth, fifth and sixth grades. Results from each of these tests are included to calculate the school Academic Index. Some districts choose to organize elementary schools to include primary through fifth grades as well as sixth, seventh, and eighth grades. Kentucky elementary schools are characterized by multiple organizational patterns based on needs determined by the local school district. Regardless 
of configuration, students at end-of-primary through eighth grade are required to participate in the KCCT. This study addressed only the scores resulting from elementary students rather than scores that would represent middle school student performance, regardless of school configuration.

Elementary school teachers from the 124 elementary schools in the GRREC region were selected as the population from which to draw the sample for the study due to the large number of possible participants. A large number of participants lend power to study results. "The larger the sample size, other things being equal, the smaller the error and the greater the reliability or precision of the results" (Cohen, 1988, p. 7.) In addition, because of the concentration of subjects assessed at fourth and fifth grade levels, it may be possible that more elementary schools rely upon the services of a teacher leader to support effective teaching for greater learning gains.

\section{Instrument}

The instrument for the study was an adaptation of the Leadership Capacity Staff Survey developed by Linda Lambert (1998). The survey provides an "assessment of leadership dispositions, knowledge, and skills needed to build leadership capacity in schools and organizations" (p. 100), based upon the theoretical framework described in Chapter Two. Survey items cluster around each of the tenets of the theoretical framework: (a) broad-based, skillful participation in the work of leadership, (b) inquirybased use of information to inform shared decisions and practice, (c) roles and responsibilities that reflect broad involvement and collaboration, (d) reflective practice/innovation as the norm, and (e) analysis of learning. Anchors for a five answer Likert-type scale include $\mathrm{NO}=$ not observed, $\mathrm{IP}=$ infrequently performed, 
$\mathrm{FP}=$ frequently performed, $\mathrm{CP}=$ consistently performed , and $\mathrm{CTO}=$ can teach to others. Lambert suggested that the survey be used as a self-assessment.

With the permission of Dr. Lambert and the Association of Supervision and Curriculum Development, the survey was adapted for one-time use in this study. Items pertinent to Kentucky teachers were added to cluster groups. Nominal survey responses were changed to numbers as noted in Appendix A to provide an interval scale of measurement.

\section{Validity and Reliability}

Research instruments must possess validity and reliability if results from their use are to be taken seriously. The survey instrument used in this study has no available data on its validity or reliability. Thus, additional steps were required prior to the use of the instrument in the actual research study.

\section{Validity}

Validity means accuracy. A research instrument possesses validity to the extent that its results are accurate, that is, that the instrument measures what it is intended to measure (Huck, 2008). Because no content validity data currently exists for the instrument to be used in this study, procedures were followed to establish it.

\section{Content Validity}

Content validity refers to the "degree to which the various items [in the instrument] collectively cover the material that the instrument is supposed to cover" (Huck, p. 89). Content validity can be established by having a panel of experts compare the items in the instrument to the known domain that the items address. "Subjective opinion" from such a panel of experts can establish the validity of the instrument. 
For this study, a panel of experts were convened to authenticate the content validity of the survey items. The panel included university professors and instructors, a district-level public school administrator, and a school principal. The panel was convened to analyze survey categories and determine if the items listed in each category are relevant to that category. Background from Lambert's (1998) research, upon which the survey items are based, was shared with the panel. In addition, the specific items added to the survey by the researcher, based on practices specific to Kentucky, were considered by the panel.

\section{Reliability}

Reliability refers to consistency of outcomes. Reliability measures the degree to which an instrument yields comparable results from comparable participants under comparable circumstances. The test-retest method was used in this study to establish reliability. Test-retest requires measuring one group of subjects twice with the same instrument, with the two testing events separated by a period of time. A correlation of the two sets of scores yields a test-retest reliability coefficient. Also referred to as the coefficient of stability because it confirms stability of results over time, the closer the coefficient is to 1.00 , the higher the reliability of the instrument (Huck, 2008).

Test-retest reliability was established in this study by recruiting participation of two graduate education classes at Western Kentucky University. With the approval of the course instructors, the first survey was administered by the researcher during class. Between one and two weeks after the initial administration of the survey, the researcher administered the instrument a second time under the same circumstances to the original participants. Based on the two sets of data, a test-retest reliability coefficient was 
calculated for instrument reliability.

\section{Procedure}

Survey administration followed a modified procedure outlined by Dillman (2000) and utilized Western Kentucky University's license for Easy Survey Package. An e-mail was sent to the Executive Director of GRREC, explaining the purpose of the study and the intent to survey elementary school teachers in the districts served by GRREC. The Executive Director was asked that the researcher be allowed to present the purpose and nature of the study to participating GRREC superintendents at a GRREC meeting. The researcher also requested permission for all elementary schools within the superintendents' individual districts to participate in the study. A permission form was made available for superintendents to sign. After permission from district superintendents is obtained, an e-mail was sent to elementary school principals in the districts where approval had been granted to outline the purpose and nature of the study. Principals were asked to forward the e-mail invitation to participate in the survey to all faculty members. The e-mail included the survey web address and requested completion of the online survey within seven days.

One week after the first e-mail to principals, a second e-mail was sent to the same group with the request that the message be forwarded to all faculty members. This e-mail expressed appreciation for initial responses and asked again that teachers participate in the online survey within the next seven days.

If adequate survey responses had not been received after 14 days, a third e-mail request would be sent to elementary school principals in districts where superintendents had given permission for participation. The e-mail thanked principals and teachers for 
their assistance and invited teacher participation in the online survey one last time.

Principals were asked to forward this message to all faculty members. A follow-up e-mail was sent to superintendents expressing gratitude for their support. An e-mail was also sent to principals to be forwarded to teachers thanking them for their participation in the survey (Dillman, 2000).

\section{Data Analysis}

Data analysis occurred through use of the Statistical Package for the Social Sciences (SPSS) software program. Multiple regression analysis was applied to survey data to determine which interactions between teachers and teacher leaders best predict student achievement, after controlling for demographic variables. Descriptive data for survey participants was also reported.

\section{Hypothesis}

The research hypothesis for the research question is predictive.

\begin{tabular}{|c|c|c|c|c|c|c|}
\hline $\begin{array}{l}\text { Teacher leader } \\
\text { interactions } \\
\text { with teachers }\end{array}$ & $\longrightarrow$ & $\begin{array}{l}\text { Effective } \\
\text { classroom } \\
\text { instruction }\end{array}$ & $\longrightarrow$ & $\begin{array}{l}\text { Improved } \\
\text { student } \\
\text { learning }\end{array}$ & $\longrightarrow$ & $\begin{array}{c}\text { Higher } \\
\text { KCCT } \\
\text { scores }\end{array}$ \\
\hline
\end{tabular}

Figure 6. Predictive model for research hypotheses.

Null Hypothesis: Teacher leader interactions with teachers have no effect on average test scores for Kentucky elementary schools on the KCCT.

\section{Summary}

This research study was a quantitative, predictive analysis of survey data and scores from the KCCT. The dependent variable was student achievement as measured by the $2008 \mathrm{KCCT}$ academic indices (AI) of elementary schools in the GRREC service region. The independent variables included five categories of teacher leader interactions 
with teachers as identified by Lambert (1998). These variables include activities clustered around (a) broad-based and skillful participation in the work of leadership; (b) inquirybased use of information to inform shared decisions and practice; (c) roles and responsibilities that reflect broad involvement and collaboration; (d) reflective practice/innovation as the norm; and (e) analysis of learning. A group of specific behaviors enacted by teacher leaders characterized each of the independent variables. Because ethnicity and socioeconomic status have been shown to influence test scores in Kentucky, controls for these factors were established.

The study addressed the following research question: What is the relationship between (a) interactions between teacher leaders and teachers, and (b) test scores for Kentucky schools on the KCCT after controlling for demographic factors known to predict average school test scores? A panel of experts established content validity for the survey. A test-retest procedure established reliability. Forward step-wise multiple regression was used for data analysis. 


\section{CHAPTER IV}

\section{RESULTS}

\section{Introduction}

The purpose of this study was to measure the relationship between teacher leader interactions with teachers and student achievement as measured by the Kentucky Core Content Test (KCCT), while controlling for demographic factors known to negatively impact student test scores. The central research question for the study sought to determine the following: What is the relationship between interactions between (a) teacher leaders and teachers and (b) test scores for schools on the KCCT, after controlling for demographic factors known to negatively impact student test scores?

The five independent variables consisted of categories of teacher leader interactions with teachers as identified by Lambert (1998). These variables included activities clustered around: (a) broad-based and skillful participation in the work of leadership; (b) inquiry-based use of information to inform shared decisions and practice; (c) roles and responsibilities that reflect broad involvement and collaboration;

(d) reflective practice/innovation as the norm; and, (e) analysis of learning. Lambert developed survey items around each construct. The researcher adapted those survey items as well as a construct name with the permission of Dr. Lambert and the Association of Supervision and Curriculum Development (ASCD). Other independent variables included socioeconomic status, measured by percentage of free and reduced lunch data 
for schools in 2008 (Nutrition Health Services, 2008), and minority population, as identified by percentage of African American enrollment for schools in 2008 (Superintendent Average Attendance Report, 2008).

The dependent variable was the 2008 KCCT score for each participating elementary school. The Academic Index (AI) for each school was used as the KCCT score. In Kentucky schools, the Academic Index (AI) is a composite of scores earned for tested content areas in Grades Three through Six.

Study participants consisted of a sample of elementary teachers in the Green River Regional Education Cooperative (GRREC) service region who taught in their current school (2008-09) during the 2007-08 academic year $(N=573)$. Participants completed the online survey instrument based on Lambert's (1998) constructs about their interactions with teacher leaders during the 2007-2008 school year.

The research hypothesis was tested using multiple regression. Percents of 2007 2008 school free and reduced lunch data and African American population were entered first into the regression formula to account for variance on the school Academic Index, the dependent variable. Any remaining variance was then attributed to the other five independent variables.

\section{Validity and Reliability}

The survey instrument used in this study had no available validity or reliability data. Therefore, processes to establish both were conducted prior to formal data gathering for the research study. A panel of experts verified content validity. Reliability was confirmed through a test/re-test procedure. 


\section{Content Validity}

A panel of experts reviewed survey items to determine if each one addressed the construct it was intended to measure. The panel included Dr. Vicki Metzgar, Dr. Molly Wilson, and Dr. Mary Evans. Dr. Metzgar is an Assistant Professor in the Department of Curriculum and Instruction at Western Kentucky University. Her dissertation research focused on teacher leadership. Dr. Molly Wilson serves as Elementary Instructional Supervisor, English Language Learner (ELL) Coordinator, and Federal Programs Director for Warren County Public Schools in Bowling Green, Kentucky. Dr. Wilson works with building level teacher leaders throughout the school district to implement Title I and English Language Learner (ELL) programs. Dr. Mary Evans is the principal of Cumberland Trace Elementary School in the Warren County Public School system. Dr. Evans moves her faculty toward opportunities for meaningful teacher interactions for continuous student progress. Teachers who work with Dr. Evans have earned national awards: (a) the 2008 American Star of Teaching Award, (b) the 2002 Kentucky Elementary Teacher of the Year, and (c) the 2008 Kentucky Teacher Hall of Fame. Dr. Evans has been recognized as the 2007 Outstanding Administrator Award, Kentucky Art Education Association. All three women have earned terminal degrees and understand the research process as well as the teacher leadership concept.

After agreeing to serve on the panel, each of the three members was sent a copy of the survey instrument with an explanation of each of the five constructs and directions for determining the degree to which each survey item addressed the construct (Appendix A). Panel members completed the content validity instrument independently and returned them to the researcher. Responses for each item were recorded and compared and 
comments regarding overall construct questions were noted. In general, if two of the three panel members marked an item as problematic for any reason, the item was revised, deleted, or combined with another survey item, depending on the panel members' accompanying comments, as well as the researcher's understanding of the item's intent. The final revision of the survey used in the study can be found in Appendix B.

\section{Reliability}

A test/re-test procedure was used to establish survey reliability. Students from two graduate level education classes $(n=26)$ were invited to complete the survey instrument at two separate times, seven days apart. The professor of record administered the surveys to the students during class time. Instrument directions made clear that survey results would be used for the sole purpose of establishing reliability. Students were free to choose not to participate.

Individual test/re-test item correlations ranged from 0.537 to 0.897 . Construct correlations were higher, ranging from 0.871 to 0.935 (Appendix C). Correlations were judged sufficiently high enough for survey item and construct reliability.

\section{Procedure}

Survey administration followed procedures outlined by Dillman (2000) and used Western Kentucky University's license for Easy Survey Package. An e-mail was sent to the Executive Director of GRREC, explaining the study and the intent to survey public school elementary teachers in the districts served by GRREC. Permission was requested to present the purpose and nature of the study to participating GRREC superintendents at the next GRREC meeting. The Executive Director granted permission and the researcher introduced the study and requested permission to survey public school elementary 
teachers in GRREC superintendents' districts. Superintendents in attendance who were willing to have elementary teachers $(N=2526)$ in the schools $(N=83)$ within their districts $(N=23)$ participate in the study signed permission forms (Appendix D).

An e-mail was sent to principals in districts where superintendents had given consent, first acknowledging the superintendent's permission, and then explaining the purpose and nature of the study along with the survey web address (Appendix E). Principals were asked to forward the e-mail to all teachers in the school. Teachers were asked to complete the survey within seven days. One week later, a second e-mail was sent to principals asking that teachers be invited once again to participate by forwarding the e-mail to all faculty members. The e-mail asked teachers to complete the survey within seven days.

Because of a crippling ice storm in Western Kentucky during the two-week period of the survey's administration, many areas were without power for a time and schools were closed from four to ten days, negatively impacting survey response rates. Therefore, a third e-mail was sent to principals seven days after the second contact. The third e-mail conveyed the same invitation to teachers for survey participation within the next seven days. Two principals replied that the survey had been sent out twice and that whoever wanted to respond had already done so. At this point, the upper limits of teacher participation appeared to have been reached $(n=573)$. 
Table 1

Participation Information: Teacher, Schools, and Districts

\begin{tabular}{lccr}
\hline & Possible & Frequency & $\begin{array}{r}\text { Percent } \\
\text { Participation }\end{array}$ \\
\hline Elementary Teachers & 2526 & 573 & 23.0 \\
Elementary Schools & 83 & 70 & 84.0 \\
School Districts & 23 & 21 & 91.3 \\
\hline
\end{tabular}

Table 1 shows participation data and response rates by teacher, school, and district. There were 573 teacher responses from a pool of 2526 potential participants, yielding a response rate of $22.68 \%$. Responses represented 70 of 83 contacted schools, a response rate of $84 \%$, and 21 of 23 public school districts, a participation rate of $91.3 \%$. Expanded school participation information can be found in Appendix F, and expanded district participation information in Appendix G. A list of the 2008 Academic Index for each school is located in Appendix H.

\section{Descriptive Statistics}

Study participants included elementary teachers $(n=573)$ from public elementary schools $(n=70)$ in the GRREC service region. Respondents completed an online survey about interactions with teacher leaders during the 2007-2008 academic year. Demographic information was also obtained in the survey. 
Table 2

Demographic Statistics: Age, Years of Teaching Experience, Years in Current School

\begin{tabular}{lccr}
\hline & $\mathrm{N}$ & Mean & Std. Deviation \\
\hline Age & 502 & 39.0 & 10.1 \\
Total Years Teaching Experience & 557 & 12.6 & 8.5 \\
Years in Current School & 557 & 8.0 & 6.6 \\
\hline
\end{tabular}

Table 2 shows the average age of the 502 respondents who reported that information. More participants reported total years teaching experience and the number of years worked in the current school than reported age. The average age of reporting respondents was 39.0 but the standard deviation indicates a wide range of ages within the reporting sample. The average years of teaching experience reported by 557 participants was 12.56 , with a standard deviation of 8.52 , suggesting that many teachers may be in the early stages of teaching careers. The mean number of years taught in the current school was 8.0 with a standard deviation of 6.6 , indicating that most responding teachers had not worked long at the current school. 
Table 3

Demographic Information: Gender, Ethnicity, and Education $(N=557)$

\begin{tabular}{|c|c|c|c|}
\hline & & Frequency & Percentage \\
\hline \multirow[t]{2}{*}{ Gender: } & Female & 519 & 93.2 \\
\hline & Male & 38 & 6.8 \\
\hline \multirow[t]{6}{*}{ Ethnicity: } & Caucasian & 544 & 97.7 \\
\hline & African American & 6 & 1.1 \\
\hline & Hispanic & 3 & 0.5 \\
\hline & Asian/Pacific Islander & 1 & 0.2 \\
\hline & Multiracial & 1 & 0.2 \\
\hline & Unknown & 1 & 0.2 \\
\hline \multirow[t]{4}{*}{ Education: } & Bachelors (Rank III) & 102 & 18.3 \\
\hline & Masters (Rank II & 275 & 49.4 \\
\hline & Rank I & 177 & 31.8 \\
\hline & Ed.S. & 3 & 0.5 \\
\hline
\end{tabular}

Table 3 reports gender, ethnicity, and education data from 557 respondents. The overwhelming majority of respondents were female (93.2\%) and Caucasian $(97.7 \%)$. While nearly half of those surveyed reported having earned a Master's degree $(49.4 \%)$, nearly one-third (31.8\%) had obtained a Rank I. Only three participants $(0.5 \%)$ reported having earned an Ed.S. degree, and 18\% indicated having received a Bachelor's degree. Approximately $82 \%$ of those reporting had pursued post-graduate degrees in the form of a Master's, Rank I or Ed.S. degree program. 
Survey gender data suggests that the sample of respondents is similar to the 20072008 GRREC population, which included 91.5\% Female and 8.5\% Male. Data on ethnicity and educational attainment for GRREC service area teachers were not available. Table 4

Demographic Information: Grade Level, Teacher Leader in Building $(N=557)$

\begin{tabular}{lcr}
\hline & Frequency & Percentage \\
\hline Grade Level K-3 & 355 & 63.7 \\
Grade Level 4-6 & 202 & 36.3 \\
Formal Teacher Leader in Building & 411 & 73.8 \\
No Formal Teacher Leader in Building & 146 & 26.2 \\
\hline
\end{tabular}

In Table 4, teacher participants are identified by grade level taught, either primary (K-3) or intermediate (4-6). While some respondents marked grades in both levels, the level at which the majority of classes were taught by the teacher was selected. More than half $(63 \%)$ of survey participants worked primarily with K-3 students while $36.3 \%$ spent most of their time with intermediate (4-6) students. Identified, formal teacher leaders served $73.8 \%$ of the teachers who answered survey questions, while $26.2 \%$ of the responding participants did not have a formal teacher leader in the school.

Information in Table 5 identifies the subjects taught by reporting respondents. The largest group of survey respondents (37.9\%) taught in self-contained classrooms. The next largest groups taught Reading (17.1\%). Special education teachers formed the third largest response group (15.3\%). Interestingly, Math teachers made up only $7.4 \%$ of respondents. All other subjects were each taught by fewer than $10 \%$ of participating respondents. Because of the nature of primary classrooms and because of the number of 
Table 5

Demographic Information: Subjects Taught $(N=557)$

\begin{tabular}{lcr}
\hline & Frequency & Percentage \\
\hline Art & 7 & 1.3 \\
Health & 1 & 0.2 \\
Language Arts & 25 & 4.5 \\
Library & 20 & 3.6 \\
Math & 41 & 7.4 \\
Music & 10 & 1.8 \\
PE & 8 & 1.4 \\
Practical Living & 16 & 2.9 \\
Reading & 95 & 17.1 \\
Science & 22 & 3.9 \\
Self-Contained Classroom & 211 & 37.9 \\
Social Studies & 16 & 2.9 \\
Special Education & & 15.3 \\
\hline
\end{tabular}

participants reporting teaching in K-3 settings (36.3\%), it is possible that the majority of those who identified their subject as "self-contained classroom" might be primary teachers.

\section{Research Question}

The research question for the study sought to determine the following: What is the relationship between interactions between (a) teacher leaders and teachers and (b) test 
scores for schools on the KCCT, after controlling for demographic factors known to negatively impact student test scores? The null hypothesis stated that there is no relationship between interactions between teacher leaders and teachers and test scores for schools on the KCCT. Using the Statistical Package for the Social Sciences (SPSS) for data analysis, the hypothesis was tested using hierarchical and simultaneous multiple regression. After excluding data from respondents who had taught in the current school for less than one year $(N=561)$, a correlation between all variables for all respondents was conducted to determine if relationships between variables could be identified.

The original plan was to aggregate data by school and to perform the data analysis on the aggregated data. This would have involved averaging each variable for each school and then doing a school level analysis. However, examination of the data resulted in a change of plan.

Although a total of 573 individual teachers responded, these came from 70 schools. The average number of respondents per school was 8.2 . However, the number of respondents from schools ranged from as few as one from one school to 31 from another school. Only three schools had 20 or more respondents. Any attempt to use aggregated data would have resulted in biases, due to a large amount of sampling error per school. The data for each school would have been based on a relatively small number of cases, and as a result the representativeness of data within each school would have been poor.

As a consequence with the problems of school level analysis, the researcher used individual teacher data for the analysis. However, this also entails a potential bias. There is lack of independence in the data, since data are coming from respondents who are clustered in schools. Violation of independence due to clustering of data results in 
inflation of Type I error. This means the probability of falsely claiming statistical significance is higher than the significance value chosen by the investigator (Cohen, Cohen, West, \& Aiken, 2003).

There are two reasons why, despite the potential biases of individual level analysis, the researcher decided to pursue that option. First, the problem of bias is greatest when the number of cases per cluster (i.e., school) increases (Barcikowski, 1981). However, as was stated above, there were not many schools that had large numbers of cases. The responding teachers came from a relatively large number of schools. Thus, the bias resulting from data clustering was judged to be not severe. Second, there was an exploratory nature to the current study, meaning that a motivation for the researcher was finding whatever possible relationships existed among the variables under study. If something is found using a method with bias toward finding significant outcomes, such an outcome would best be considered an indicator of a potential relationship rather than a definitive finding.

In Table 6, correlation results between variables for all participants are displayed. A moderate negative correlation is noted between School Academic Index and students receiving free and reduced lunch $(-0.643, \mathrm{p}<.01)$. The percent of African American students $(-0.280, \mathrm{p}<.01)$ was also observed as a negative correlation with school Academic Index, although not as strongly as the free/reduced lunch variable. Perhaps most surprising was the lack of significant positive correlation between an identified teacher leader in the school $(-0.039)$ and School Academic Index. Even more so was the minimal relationship between Academic Index and each of the teacher leader constructs: leadership work $(0.133, \mathrm{p}<.01)$, shared decision making $(0.112, \mathrm{p}<.01)$, collaboration 
Table 6

Correlations for All Variables for All Respondents: Academic Index, \% School African American, \% School Free/Reduced Lunch, Leadership Work, Shared Decision Making, Collaboration, Reflective Practice, Analysis of Learning, and Identified Teacher Leader $(N=561)$

\begin{tabular}{|c|c|c|c|c|c|c|c|c|c|}
\hline & $\begin{array}{l}\text { Academic } \\
\text { Index }\end{array}$ & $\% \mathrm{AA}$ & $\begin{array}{l}\% \mathrm{~F} / \mathrm{R} \\
\text { Lunch }\end{array}$ & $\begin{array}{l}\text { Leadership } \\
\text { Work }\end{array}$ & $\begin{array}{l}\text { Shared } \\
\text { Decisions }\end{array}$ & Collaboration & $\begin{array}{l}\text { Reflective } \\
\text { Practice }\end{array}$ & $\begin{array}{c}\text { Analysis } \\
\text { of } \\
\text { Learning }\end{array}$ & $\begin{array}{l}\text { Identified } \\
\text { TL }\end{array}$ \\
\hline Academic Index & 1.000 & -.280 & $-.643^{* *}$ & $.133^{*}$ & $.112^{* *}$ & $.106^{*}$ & $.119^{* *}$ & $.122^{* *}$ & .039 \\
\hline$\% \mathrm{AA}$ & & 1.000 & $.380^{* * *}$ & $-.088^{*}$ & $-.187^{* *}$ & $-.119^{*}$ & -.083 & $-.116^{* *}$ & .021 \\
\hline$\% \mathrm{~F} / \mathrm{R}$ Lunch & & & 1.000 & -.116 & $-.097^{*}$ & $-.105^{*}$ & $-.109 *$ & -.097 & $.122^{* *}$ \\
\hline Leadership Work & & & & 1.000 & $.860^{* *}$ & $.892^{* *}$ & $.896^{* *}$ & $.899 * *$ & $-.201 * *$ \\
\hline Shared Decisions & & & & & 1.000 & $.845^{* *}$ & $.846^{* *}$ & $.881^{* *}$ & $-.185^{* *}$ \\
\hline Collaboration & & & & & & 1.000 & $.910^{* *}$ & $.898^{* *}$ & $-.146 * *$ \\
\hline Reflective Pr & & & & & & & 1.000 & $.917^{* *}$ & $-.165 * *$ \\
\hline $\begin{array}{l}\text { Analysis of } \\
\text { Learning }\end{array}$ & & & & & & & & 1.000 & $-.154^{* *}$ \\
\hline Identified TL & & & & & & & & & 1.000 \\
\hline
\end{tabular}

**Correlation is significant at the 0.01 level (2-tailed). ${ }^{*}$ Correlation is significant at the 0.05 level (2-tailed). 
$(0.106, \mathrm{p}<.05)$, reflective practice $(0.119, \mathrm{p}<.05)$, and analysis of learning $(0.122$, $p<.05)$. These correlations to Academic Index were very weak. Predictors should be highly correlated with the dependent variable but only moderately coordinated with each other (Shavelson, 1988).

The decision was made to analyze sample data by grade level assignment to determine if results would differ based on grade level. End of primary (traditional Grade Three) is assessed for accountability, while Grades Four, Five, and Six are all assessed and their scores typically contribute more volume and weight collectively to the Academic Index than the end of primary assessment. For this reason, a building teacher leader may tend to have more and broader types of interactions with teachers assigned to intermediate grades. Because the school Academic Index is weighted more heavily from tests taken by Fourth, Fifth, and Sixth Grade students, limiting responses to those of intermediate teachers was considered appropriate. Therefore, after excluding data from respondents who had taught in the current school for less than 1 year, a correlation between all variables for Fourth, Fifth, and Sixth Grade respondents $(N=202)$ was conducted to determine if relationships between variables could be identified.

Table 7 displays the correlation data between variables from intermediate teacher responses. School free and reduced lunch still displayed the strongest negative relationship with Academic Index $(-.554, \mathrm{p}<.01)$, although not as strong as with all responses $(-.643, \mathrm{p}<.01)$. The number of African American students also negatively correlated with Academic Index $(-.228, p<.01)$, compared to all responses $(-.280, p<.01)$ as did an identified teacher leader in the school (-.129). An identified teacher leader had a greater negative relationship to Academic Achievement with intermediate teachers than with all teachers (-.039). However, both of these negative correlations were not statistically significant. Each of the teacher leader constructs were 
Table 7

Correlations for All Variables for 4-6 Grade Teachers: Academic Index, \% School African American, \% School Free/Reduced Lunch, Leadership Work, Shared Decision Making, Collaboration, Reflective Practice, Analysis of Learning, and Identified Teacher Leader $(N=202$

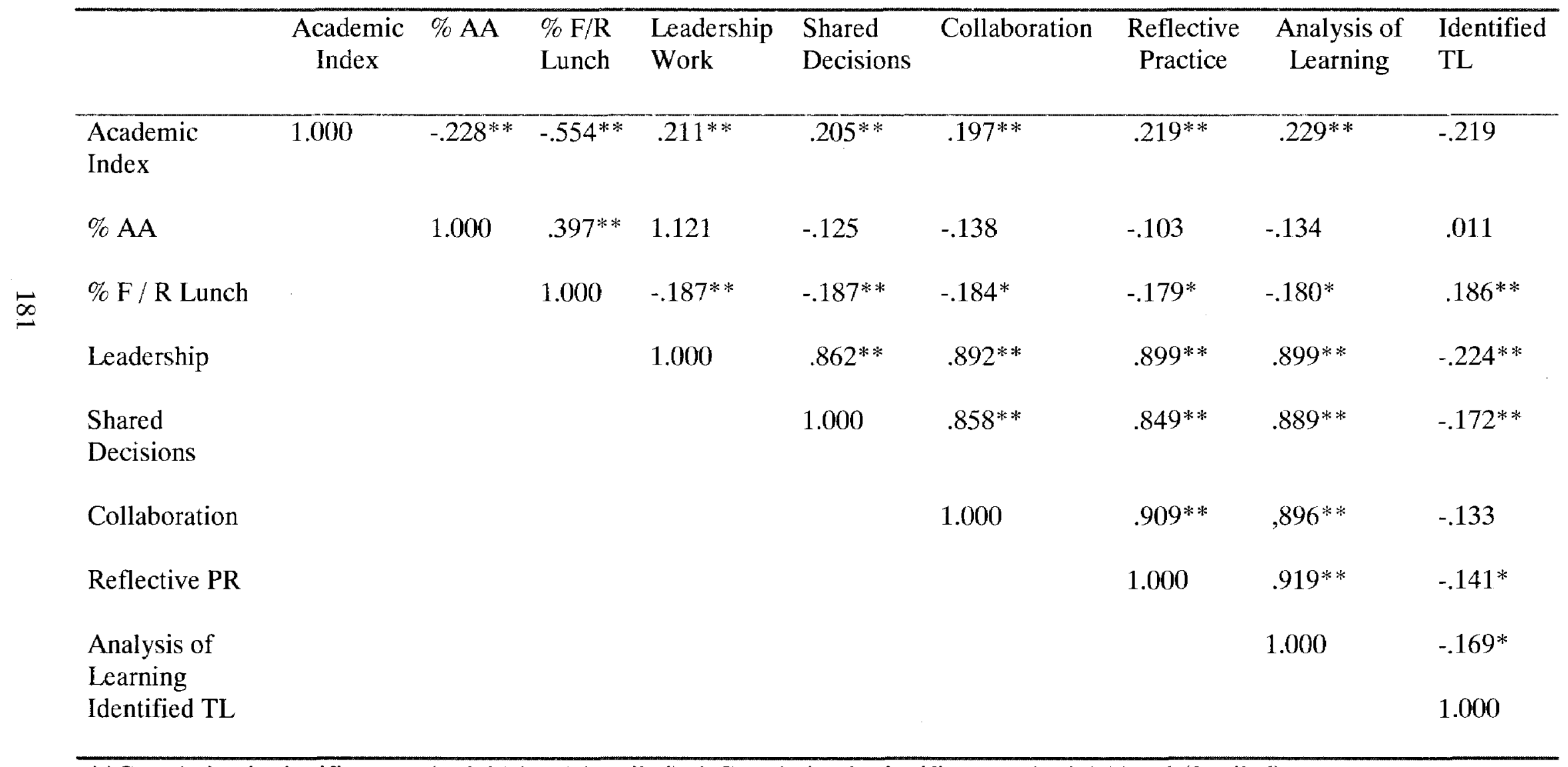

${ }^{* *}$ Correlation is significant at the 0.01 level (2-tailed). ${ }^{*}$ Correlation is significant at the 0.05 level (2-tailed). 
more strongly correlated in the intermediate teacher responses than in all responses combined: (a) leadership work $(.211, \mathrm{p}<.01)$ compared to all $(.133, \mathrm{p}<.01)$; (b) shared decision making $(.205, \mathrm{p}<.01)$ in contrast to all $(.122, \mathrm{p}<.01)$; (c) collaboration $(.197, \mathrm{p}$ $<.01)$ as opposed to all $(.106, \mathrm{p}<.05)$; (d) reflective practice $(.219, \mathrm{p}<.01)$ higher than all $(.119, \mathrm{p}<.01)$; and, (e) analysis of learning $(.229, \mathrm{p}<.01)$ compared to all responses together $(.122, \mathrm{p}<.01)$. Because the correlations were greater than or equal to 0.2 , the decision was made to conduct multiple regression analysis using only data from intermediate teacher respondents, i.e., fourth, fifth, and sixth grade teachers. Post-hoc correlations were also calculated for self-contained classroom respondents, non-selfcontained classroom participants, and K-3 teachers. In all cases, results did not yield sufficient correlation size to support a regression analysis.

Descriptive statistics for intermediate teacher responses to teacher leader constructs are shown in Table 8. The mean free and reduced lunch student population for schools of intermediate teacher respondents was 54.02 with a standard deviation of 14.86 , indicative of large numbers of low socioeconomic students. The average for intermediate teacher responses to survey questions measuring teacher leadership constructs ranged from 3.54 to 3.86 , a difference of 0.32 . The standard deviations for intermediate teacher responses to leadership constructs ranged from 1.00 to 1.1 . 
Table 8

Descriptive Statistics: Intermediate Teacher Responses to Teacher Leader Constructs

\begin{tabular}{lccr}
\hline & N & Mean & Std. Deviation \\
\hline \% School African American & 202 & 7.2 & 7.4 \\
\% School Free/Reduced Lunch & 202 & 54.0 & 14.8 \\
Leadership Work & 197 & 3.5 & 1.0 \\
Shared Decision Making & 195 & 3.8 & 1.0 \\
Collaboration & 195 & 3.6 & 1.1 \\
Reflective Practice & 195 & 3.6 & 1.0 \\
Analysis of learning & 196 & 3.6 & 1.0 \\
\hline
\end{tabular}

The forward method of variable entry, with $p=.05$ as the statistical criterion for variable entry, was used. Table 9 shows the statistically significant predictor variables that entered the regression equation based on the correlation criterion.

Table 9

Regression Summary: Teacher Leader Constructs on Student Achievement

\begin{tabular}{cccc}
\hline $\mathrm{R}$ & $\mathrm{R}$ Square & $\begin{array}{c}\text { Adjusted R } \\
\text { Square }\end{array}$ & $\begin{array}{c}\text { Std. Error of } \\
\text { the Estimate }\end{array}$ \\
\hline .550 & .303 & .296 & 7.04415 \\
\hline
\end{tabular}

Predictors: (Constant), \% School Free/Reduced Lunch, Analysis of Learning Dependent Variable: School KCCT Index for 2008

For intermediate teacher respondents, the percentage of free and reduced lunch and the analysis of learning construct resulted in $R^{2}=.303$ with Adjusted $R^{2}=.296$. The analysis of learning construct and percentage of free and reduced lunch accounted for nearly $30 \%$ of the variance in student achievement as measured by the School Academic Index, a large effect size. The ANOVA for the regression equation was significant, $F(2$, 
$191)=41.506, \mathrm{p}<.01$

Table 10 shows beta coefficients and $t$ scores for the predictor variables in the final equation. Only percentage of free and reduced lunch $(\mathrm{p}<.01)$ and analysis of learning $(\mathrm{p}<.05)$ significantly predicted student achievement as measured by KCCT School Academic Index. While percentage of free and reduced lunch had a negative coefficient $(\beta=-.512)$, the student analysis of learning scale score had a positive relationship $(\beta=.128)$.

Table 10

Predictor Variables for School Academic Index

\begin{tabular}{lrrrrr}
\hline & $B$ & $S E B$ & Beta & $t$ & Sig. \\
\hline (Constant) & 106.853 & 2.916 & & 36.649 & .000 \\
$\% \mathrm{~F} / \mathrm{R}$ & -.296 & .036 & -.512 & -8.321 & .000 \\
$\begin{array}{l}\text { Analysis of } \\
\text { Learning }\end{array}$ & 1.035 & .496 & .128 & 2.087 & .038 \\
\hline
\end{tabular}

Dependent Variable: School KCCT Index for 2008

The analyses for survey responses regarding the analysis of learning construct are shown on Table 11. The data illustrate the frequencies at which participants responded to the analysis of learning interaction items with the two highest possible ratings on the survey Likert scale: $4=$ Consistently Performed, and $5=$ An Established Behavior. None of the combined Level 4 and Level 5 responses for any one item total less than $50 \%$, indicating that these interactions were common between many teachers and teacher leaders. Items with the largest total combined Level 4 and Level 5 responses (over 60\%) included numbers $43,33,35,34$ and 38 . Item 43 ranked the highest with a combined 
Table 11

Frequency of Level 4 and Level 5 Responses to Analysis of Learning Construct Items $(N=202)$

\begin{tabular}{ccccc}
\hline Analysis of Learning Survey Item & Frequency: & Valid & Frequency: & Valid \\
& Consistently & Percent & Established & Percent \\
& Performed & & Behavior
\end{tabular}

33. Works with others to develop criteria to monitor and assess implementation of

62

31.6

64

32.7

school-wide programs.

34. Works with others to develop criteria

54

27.6

68

34.7

for individual and shared accountability

for student learning.

35. Works with others to establish

63

32.1

64

32.7

rigorous expectations for all students.

36. Designs, teaches, coaches, and

55

28.1

57

29.1

evaluates curriculum.

37. Designs, teaches, coaches, and

28.6

48

24.5

evaluates instruction.

38. Designs, teaches, coaches, and

65

33.2

56

28.6

evaluates assessment.

39. Implements a system for regular,

62

32.0

43

22.2

meaningful feedback to students and

parents about student progress.

40. Provides meaningful feedback to

60

30.6

54

27.6

colleagues.

41. Accepts feedback from students and

61

31.6

47

24.4

parents and amends practice when

appropriate.

42. Accepts feedback from colleagues

63

32.5

47

24.2

and amends practice when appropriate.

43. Ensures that evidence of learning

68

34.9

63

32.3 includes assessment of data and analysis of student work. 
Level 4 and Level 5 response rate of $67 \%$. Items 33 and 35 tied with a Level 4 and Level 5 response rate of $64 \%$. Items 34 and 38 also tied with a combined Level 4 and Level 5 response rate of $61 \%$. These five items suggest the types of interaction between teachers and teacher leaders that may predict student achievement.

Based on the results of the correlation matrix, regression analysis, ANOVA, and $t$ test, two variables (percentage of free and reduced lunch and the analysis of learning construct for teacher leadership) exhibited a statistically significant relationship to 2008 school KCCT scores as measured by the Academic Index. Therefore, the null hypothesis was rejected. Teacher leader interactions with teachers that deal with analysis of learning have a positive predictive relationship with student achievement as measured by school KCCT scores.

\section{Summary}

The purpose of this study was to measure the relationship between teacher leader interactions with teachers and student achievement as measured by the Kentucky Core Content Test (KCCT), while controlling for demographic factors known to negatively impact student test scores. The central research question for the study sought to determine: What is the relationship between interactions between (a) teacher leaders and teachers and (b) test scores for schools on the $\mathrm{KCCT}$, after controlling for demographic factors known to negatively impact student test scores?

The five independent variables consisted of categories of teacher leader interactions with teachers as identified by Lambert (1998). These variables included activities clustered around (a) broad-based and skillful participation in the work of leadership; (b) inquiry-based use of information to inform shared decisions and practice; 
(c) roles and responsibilities that reflect broad involvement and collaboration;

(d) reflective practice/innovation as the norm; and, (e) analysis of learning. Two additional independent variables known to negatively impact student achievement included socioeconomic status, measured by percentage of free and reduced lunch data for 2008 , and minority population, identified by percentage of 2008 school African American enrollment.

Step-wise forward multiple regression analysis was used to determine if the teacher leadership construct variables impacted student achievement as measured by the School Academic Index. A statistically significant relationship existed between one of the teacher leadership constructs, analysis of learning, and the School Academic Index. 


\section{CHAPTER V \\ DISCUSSION AND CONCLUSIONS}

Introduction

Teacher leadership has been a topic of interest in public schools and research circles since the 1980's. Multiple studies trace the evolution of the purpose and practice of teacher leadership. Bringing teachers to the table in school-based decision-making roles was intended to ignite changes in school-wide instructional practice (Griffin, 1995; Taylor \& Bogotch, 1994) and student achievement (Smylie et al., 1996; Stone et al., 1997; Taylor \& Bogotch). Those goals were not achieved (Cameron, 2005; Griffin; Smylie, 1992a). The culture of teacher autonomy and distrust of colleagues who would lead change rendered this level of teacher leadership ineffective for student achievement gains.

Later research chronicled and analyzed the teacher leadership experience of classroom teachers who worked as full-time classroom teachers, but who also influenced teacher colleagues and performed additional duties related to instructional leadership. These individuals viewed their task as supporting the instructional practice of teachers in the school, sharing knowledge and insight about instructional practice, and influencing school-wide decisions (Beattie, 2002; Cruz, 2003; Frost \& Durrant, 2002; Hatch et al., 2005; Little, 1995; Mangin, 2005; Sawyer, 2001, Silva et al., 1996; Smylie \& Denny, 1990; Spillane et al., 2003). Ironically, these teacher leaders spent less time interacting 
with peers about teaching practices (Smylie \& Denny) than in any other leadershiprelated activity. The qualitative methods used in most of these research studies described the experiences and practices of classroom teachers who also served as teacher leaders, but did not add new information to the literature about the extent to which teacher leaders impacted instruction and student achievement.

Likewise, teacher leadership viewed through the lens of distributed leadership theory and practice yielded similar results. Principals mentored teacher leaders to provide leadership for change through participation in shared redundant tasks, inviting teachers to share creatively in the work and responsibility of leadership with the intent of improving instructional performance (Heller \& Firestone, 1995; Muijs \& Harris, 2006) and student learning (Camburn et al., 2003; Chrispeels \& Martin, 2002). The research offered descriptions of how distributed leadership could be enacted with effective principals and skillful teacher leaders to improve teaching and learning, but no statistical data confirmed the relationship between teacher leadership and student achievement.

More recent studies examined the effect of principal and teacher leadership on student engagement or student outcomes (Leithwood \& Jantzi, 1999, 2000; Silins \& Mulford, 2004). Neither student engagement nor student outcomes were defined as student achievement, but both were expected to improve student achievement. The research results suggested that principals exerted a small significant impact on student engagement and that teacher leaders exerted none at all.

The issue that prompted this study was the limited quantitative data regarding the effects of teacher leadership on student achievement. If the concern of teacher leadership is, as Pellicer and Anderson (1995) state, "teachers helping teachers so that teachers can, 
in turn, better help students" (p. 22), then measureable student achievement data should support that premise. Few studies have tested teacher leadership to determine if it has any relationship to student achievement. Two studies that have tested it (Wenglinsky, 2002; Jantzi, 2006) used student achievement data, but the studies were flawed or were considered unstable. The problem with measuring the effect of teacher leadership lies in the research design. Establishing a causal relationship between teacher leadership and student achievement would require complex research that would involve multiple mediating and moderating variables that might influence student learning and $\backslash$ achievement. Many of those variables would be difficult to quantify. The research that is needed to establish a causal relationship would be timely, costly, and statistically messy. In addition, withholding treatment from students to establish such a relationship could likely be unethical.

Meanwhile, schools and districts "increasingly recognize teacher leadership as a strong and pervasive trend" (Clemson-Ingram \& Fessler, 1997, p. 104), even though the trend has not been supported with quantifiable data. Of the 557 teachers from 70 schools in the Green River Regional Education Cooperative (GRREC) service region who returned responses to the survey statement "An identified teacher leader (i.e., curriculum coordinator, curriculum specialist) is employed at my school," $73.8 \%$ answered in the affirmative. Formal teacher leader positions have become normative in the Western Kentucky school districts served by GRREC. Yet, "the near absence of attention to accountability issues in the teacher leadership is difficult to justify" (Murphy, 2005). This study asks if there is a relationship between teacher leadership interactions with teachers and student achievement, specifically in elementary schools in the GRREC region. 


\section{Summary of the Study}

Subjects in this non-experimental, quantitative study were teachers from public elementary schools in Kentucky in the GRREC service region who taught at their current school during the 2007-2008 academic year. Data was gathered through an online survey. Participants shared demographic information as well as responses to survey questions about their interactions with teacher leaders during the 2007 - 2008 school term. Survey questions, adapted from the work of Lambert (1999), measured five teacher leadership constructs: (a) broad-based and skillful participation in the work of leadership; (b) inquiry-based use of information to inform shared decisions and practice; (c) roles and responsibilities that reflect broad involvement and collaboration; (d) reflective practice/innovation as the norm; and, (e) analysis of learning.

Multiple regression was used for data analysis to answer the research question: What is the relationship between interactions between (a) teacher leaders and teachers and (b) test scores for schools on the Kentucky Core Content Test (KCCT), after controlling for demographic factors known to negatively impact test scores?

The two demographic factors included socioeconomic status, measured by percentage free and reduced lunch data for 2008 , and minority population, identified by percentage of African American enrollment in 2008. In the regression model, the predictor variables included the following: (a) percentage of free and reduced lunch, (b) percentage of African American enrollment, (c) leadership work, (d) shared decision making, (e) collaboration, (f) reflective practice, and (g) analysis of learning. The criterion variable was KCCT test scores as measured by the 2008 School Academic Index for each school. Descriptive statistics were also calculated for teacher participants. 


\section{Discussion and Implications}

The null hypothesis for the research question stated that teacher leader interactions with teachers have no effect on test scores for Kentucky elementary schools on the KCCT. This hypothesis was tested using a multiple regression research design. Pearson correlations were calculated between the predictor variables (percentage of free and reduced lunch, percentage of African American enrollment, leadership work, shared decision making, collaboration, reflective practice, analysis of learning) and the criterion variable, 2008 School Academic Index (Table 6). The strong negative correlation between School Academic Index and percentage of free and reduced lunch students was not surprising, nor was the smaller negative relationship between School Academic Index and percentage of African American students. All other correlations were too small to support a regression analysis. One possible reason could be that $63.7 \%$ of the respondents were K-3 teachers. Of all the primary levels, only third grade students are assessed for state accountability purposes. However, all fourth, fifth, and sixth grade students are assessed and their scores contribute more volume and weight collectively to the Academic Index than the end of primary assessment. For this reason, it is possible that a building teacher leader may have more interactions with intermediate grade teachers. It is possible that fewer instances of involvement by building teacher leaders with primary teachers (the majority of survey participants) could have impacted total results. A more balanced participation rate between primary and intermediate teachers may yield stronger correlation data. Future studies could survey equal numbers of participants from each level and compare the outcomes to those of this study.

More interesting than the minimal correlations between the predictor variables 
and criterion variable for all respondents was the negative relationship (-.039) between the School Academic Index and the presence of an identified teacher leader in the school. Previous research noted the strong cultural norm of individual teacher autonomy as a primary reason for teacher reluctance to challenge peers in decision making for school change (Cameron, 2005; Griffin, 1995; Smylie, 1992a). Little (1995) as well as Smylie and Denny (1990) noted that teachers sometimes presented the greatest impediment to the success of teacher leaders. In this study, however, the possible reasons for the negative relationship between building teacher leaders and School Academic Index are not obvious. The fact that these results support previous findings (Leithwood \& Jantzi, 1999, 2000; Silins \& Mulford, 2004) where teacher leadership had no impact on student engagement or student outcomes appears important for district and school policy makers to note. However, a caveat to this statement is also in order. The mean percentage of students receiving free and reduced lunch $(54 \%)$ in schools with participating intermediate teachers (see Table 8) indicates a large population of lower socio-economic students. The presence of a formal teacher leader yielded a small positive correlation (.186) to the percentage of students receiving free and reduced lunch (see Table 7). This suggests that participating schools with a large free and reduced lunch student population are slightly more likely to have a formal teacher leader. These teacher leaders may provide important support to enhance the instructional capacity of schools with high numbers of at-risk students.

Because the correlations between the predictor and criterion variables based on all responses were too small (less than 0.2 ) to conduct a regression analysis, additional correlations were tested with participant sub-groups (self-contained classroom teachers, 
non-self-contained classroom teachers, K-3 teachers, 4-6 teachers) to determine if stronger relationships existed between the predictor and criterion variables within other groups. Only the correlations for the intermediate teacher sub-group (Table 7) were greater than or equal to 0.2 , and considered sufficient to calculate a multiple regression analysis. However, the unexpected outcome was the larger negative relationship between School Academic Index and the presence of a formal teacher leader in the building for the intermediate teacher respondents (-.129) than the correlation for the same two variables for all respondents (-.039). The reasons for the higher negative correlation can be hypothesized but the data from this study do not explain why this relationship exists. Once again, previous research regarding the absence of relationship between teacher leadership and student engagement and student outcomes (Leithwood \& Jantzi, 1999, 2000; Silins \& Mulford, 2004) is supported. Even more so, correlation data in this study suggest that having a formal teacher leader is not a positive predictor of student achievement.

The subsequent regression analysis for predictor variables and the criterion variable using only intermediate teacher survey responses yielded significant results. The control variable of percentage of free and reduced lunch and the analysis of learning construct together accounted for $30 \%$ of the variance in student achievement as measured by the School Academic Index of the KCCT, a large effect size. As expected, the percent of free and reduced lunch negatively correlated to the School Academic Index $(\beta=-.512)$, while the analysis of learning construct exerted a positive effect $(\beta=.128)$. The significant relationship of the analysis of learning variable to 2008 school KCCT scores as measured by the School Academic Index prompts closer scrutiny of the survey 
items which measured the analysis of learning construct. Table 11 highlights the analysis of learning interactions which were most frequently experienced by intermediate teacher respondents. The highest scoring items included the following: (a) ensures that evidence of learning includes assessment of data and analysis of student work; (b) works with others to develop criteria to monitor and assess implementation of school-wide programs; (c) works with others to establish rigorous expectations for all students; (d) works with others to develop criteria for individual and shared accountability for student learning; and (e) designs, teaches, coaches, and evaluates assessment. The significant results of this regression analysis have implications for schools and districts, as well as for university teacher education programs.

The first implication deals with professional development. A needs assessment based on these analysis of learning survey items could determine levels of the staff's knowledge, understanding, and implementation of each item at the school level. The assessment could first address those analysis of learning survey items that received the largest volume of the highest rankings from the research study participants. From the needs assessment results, goals for job-embedded learning for all teachers could be set and prioritized. Both short-term and long-term professional development plans could be designed for each school that could positively impact student achievement. District leadership could support these plans by providing time and money for training and modeling analysis of learning interactions. The goal would be common, skillful practice by all teachers of the five top-ranking behaviors described in the survey.

The second implication concerns school and district administrators and building teacher leaders. While correlation data from the study indicated a negative relationship 
between formal teacher leader positions and School Academic Indices, the regression analysis suggested that specific types of leadership activities, i.e., those related to analysis of learning, were significant in predicting Student Academic Index. If school and district administrators employ building level teacher leaders, the job description for the position should include the tasks and behaviors described within the analysis of learning survey items. The roles and responsibilities of teacher leaders should be streamlined to clearly focus on activities known to predict improvement in student achievement.

The third implication concerns how teacher leadership is enacted in schools. Results from this study indicate a negative correlation between having a formal teacher leader in a school and the School Academic Index, yet interactions between teachers and formal teacher leaders around analysis of learning was a significant predictor of the School Academic Index. A possible solution to this paradox is posed by one of the underlying theoretical constructs of this study. Situated learning (Lave \& Wenger, 1991) involves becoming a member of a community of practice, experiencing learning as a social practice involving co-participants who learn by doing. Communities of practice, later termed professional learning communities (Dufour \& Eaker, 1998; Dufour et al., 2005; Dufour et al., 2006; Hord, 1997), were found to improve instructional capacity in the classroom and gains in student achievement (AISR, 2004). These findings, along with the results of this study, support the expansion of the concept and practice of moving from one formal teacher leader in a school to the practice of developing shared leadership among all teachers in professional learning communities. Interactions around analysis of learning described in this study could occur between groups of teachers trained in those skills instead of between one teacher leader and all teachers. This change would require 
that all teachers assume responsibility for thinking and learning about how together they can improve instruction to provide continuous academic growth for all students.

The final implication of this study pertains to university teacher education programs. In re-designing teacher education programs, multiple opportunities should be provided, from the pre-service through the graduate level, for candidates to develop the knowledge and skills described in the analysis of learning survey construct items. Universities and school districts should partner to provide teachers with a clear, consistent process for becoming an effective professional learning community member.

Suggestions for Future Research

Future research could replicate this study using different population samples to determine if outcomes would be similar. Middle school teachers and high school teachers in the GRREC region could be studied separately to see if the results supported the findings of this study. Elementary schools in other Kentucky educational cooperative service regions could be surveyed to see if results from the GRREC region were supported. This study did not have a balanced response rate from targeted grade levels, perhaps due to the unfortunate weather circumstances at the time the survey invitation was extended. A second study could be conducted with the same schools, targeting only intermediate teachers or only primary teachers. Due to the federal and state emphasis on Reading and Math achievement and the attention that all schools are focusing on continuous student improvement in these skills, surveying only elementary Reading and Math teachers may yield more pertinent results. The study could also be replicated with Title I schools employing full-time teacher leaders. Such a study over an extended period of time may yield information about the relationship of teacher leaders to growth in 
student achievement, a topic not addressed in this study.

Further research is also needed to compare the relationship of each of the two teacher leadership models (formal teacher leader model and professional learning community model) to student achievement. While current research appears to support the effectiveness of the professional learning community model for student achievement gains, the relationship has not yet been established using Kentucky achievement data. Additional studies using Lambert's (1998) leadership survey could enhance the reliability and usefulness of the instrument.

Finally, minimal quantitative literature exists regarding the relationship between teacher leaders and student achievement and principals and student achievement. More research is needed to uncover the chain of variables whose anchor rests with the school principal and leadership team, winds through every teacher's classroom, and leads to every student's achievement.

\section{Conclusion}

This study examined the relationship between teacher leader interactions with teachers and student achievement as measured by KCCT scores. A survey, adapted from the work of Lambert (1998), measured five teacher leadership constructs: (a) broad-based and skillful participation in the work of leadership; (b) inquiry-based use of information to inform shared decisions and practice; (c) roles and responsibilities that reflect broad involvement and collaboration; (d) reflective practice/innovation as the norm; and, (e) analysis of learning. The percentage of free and reduced lunch for 2008 and the percentage of African American students for 2008 were variables also considered due to their known negative impact on student achievement. 
Formal teacher leader positions became more common in Kentucky after the Kentucky Education Reform Act of 1990 and annual high stakes accountability testing became reality for all Kentucky schools. Many districts recognized the need to support overworked principals in their role as instructional leaders and the research on teacher leadership pointed to a solution. Building-level teacher leaders could support and enhance the instructional improvement efforts of the principal. As the position became the norm in many Kentucky schools, quantitative research from studies conducted in schools located in other states was published which suggested that teacher leadership had no measurable effect on student achievement.

This study sought to determine if any relationship existed between teacher leadership constructs and student achievement measured by Kentucky's specific School Academic Index. While additional research is needed to determine if the professional learning community model is more effective than the individual teacher leadership model, results from this study suggest that specific interactions between teachers and teacher leaders around analysis of learning constructs are significant in predicting student achievement as measured by the KCCT School Academic Index. 


\section{REFERENCES}

Andersen, K. D. (2004). The nature of teacher leadership in schools as reciprocal influences between teacher leaders and principals. School Effectiveness and School Improvement, 15, 97-113.

Annenberg Institute for School Reform. (2004). Professional learning communities. Retrieved June 21, 2008 from http://www.annenberginstitute.org/pdf/ProfLearning.pdf

Barcikowski, R. S. (1981). Statistical power with group means as the units of analysis. Journal of Educational Statistics, 6, 267-285.

Barth, R. S. (2001). Teacher leader. Phi Delta Kapan, 82, 443-449.

Beachum, F., \& Dentith, A. M. (2004). Teacher leaders creating cultures of school renewal and transformation. The Educational Forum, 68, 276-286.

Beattie, M. (2002). Educational leadership: Modeling, mentoring, making and re-making a learning community. European Journal of Teacher Education, 25, 199-221.

Birky, G., \& Ward, C. (2002). Perspectives of teacher leaders in an educational reform environment: Finding meaning in their involvement. ScholarPractitioner Quarterly, 2, 9-22.

Bogdan, R.C., \& Biklen, S.K. (2003). Qualitative research for education: An introduction to theory and methods. Boston: Pearson Education, Inc.

Brown, J.S., Collins, A., \& Duguid, P. (1999). Situated cognition and the culture 
of learning. Educational Researcher: A Publication of the American Educational Research Association, 18, 32-42.

Brown, K., \& Anfara Jr., K. (2002). The walls of division crumble as ears, mouths, minds and hearts open: A unified profession of middle-level administrators and teachers. International Journal of Leadership in Education, 5, 33-49.

Camburn, E., Rowan, B., \& Taylor, J.E. (2003). Distributed leadership in schools: The case of elementary schools adopting comprehensive school reform models. Educational Evaluation and Policy Analysis, 25, 347-373.

Cameron, D. H. (2005). Teachers working in collaborative structures. Educational Management Administration \& Leadership, 33, 311-330.

Cheng, Y. C. (1994). Teacher leadership style: A classroom-level study. Journal of Educational Administration, 32, 54-71.

Chrispeels, J. H., \& Martin, K. J. (2002). Four school leadership teams define their roles within organizational and political structures to improve student learning. School Effectiveness \& School Improvement, 13, 327-365.

Cohen, J. (1988). Statistical power analysis for the behavioral sciences. Hillsdale, NJ: Lawrence Erlbaum Associates.

Cohen, J., Cohen, P., West, S.G., \& Aiken, L.S. (2003) Applied multiple regression/correlation analysis for the behavioral sciences $\left(3^{\text {rd }}\right.$ ed.). Mahwah, NJ: Lawrence Erlbaum.

Clemson-Ingram, R., \& Fessler, R. (1997). Innovative programs for teacher leadership. Action in Teacher Education, 29(4), 877-908. 
Creswell, J. W., \& Miller, D.L. (2000). Determining validity in qualitative inquiry. Theory into Practice, 39(3), 124-130.

Crowther, F., \& Olsen, P. (1997). Teachers as leaders: An exploratory framework. International Journal of Educational Management, 11(1), $6-13$.

Cruz, D. (2003). Teacher leaders: Middle school mathematics classrooms. Research in Middle Level Education Online, 26(2), 10-27.

Darling-Hammond, L. (1993). Teacher leadership for creating innovative schools. In C.L. Fagnano \& K. N. Hughes (Eds.), Making schools work: A view from the firing lines (pp. 47-64). Boulder, Westview.

Datnow, A., \& Castellano, M. E. (2001). Managing and guiding school reform: leadership in success for all schools. Educational Administration Quarterly, 37, 219-249.

Denzin, N.K. (1989). The research act: A theoretical introduction to sociological methods. Englewood Cliffs, NJ: Prentice Hall.

Dillman, D.A. (2000). Mail and internet surveys: The tailored design method $\left(2^{\text {nd }}\right.$ ed.). New York: John Wiley \& Sons, Inc.

Dufour, R., \& Eaker, R. (1998). Professional learning communities at work. Bloomington: Solution Tree.

Dufour, R., Eaker, R. \& Dufour, R. (Eds.) (2005). On common ground. Bloomington: Solution Tree.

Dufour, R., Dufour, R., Eaker, R., \& Many, T. (2006). Learning by doing: A handbook for professional learning communities at work. Bloomington: 
Solution Tree.

Foster, R. (2004). Leadership and secondary school improvement: Case studies of tensions and possibilities. International Journal of Leadership in Education, 8, 35-52.

Frost, D., \& Durrant, J. (2002). Teachers as leaders: Exploring the impact of teacher-led development work. School Leadership and Management, 22, 143-161.

Griffin, G. A. (1995). Influences of shared decision making on school and classroom activity: Conversations with five teachers. The Elementary School Journal, 96, 29-45.

Gronn, P. (2002). Distributed leadership as a unit of analysis. The Leadership Quarterly, 13, 423-451.

Grubb, W. N., \& Flessa, J. J. (2006). "A job too big for one": Multiple principals and other nontraditional approaches to school leadership. Educational Administration Quarterly, 42, 518-550.

Harris, A. (2002). Effective leadership in schools facing challenging contexts. School Leadership and Management, 22, 15-26.

Harris, A. (2005). Teacher leadership: More than just a feel-good factor? Leadership \& Policy in Schools, 4, 210-219.

Hart, A.W. (1994). Creating teacher leadership roles. Educational Administration Quarterly, 30, 472-497.

Hatch, T., White, M., \& Faigenbaum, D. (2005). Expertise, credibility, and influence: How teachers can influence policy, advance research, and 
improve performance. Teachers College Record, 107, 1004-1035.

Heller, M., \& Firestone, W. (1995). Who's in charge here? Sources of leadership for change in eight schools. The Elementary School Journal, 96, 65-86.

Henning, J. E. (2006). Teacher leaders at work: Analyzing standardized achievement data to improve instruction. Education, 126, 729-737.

Herzberg, F., Mausner, B., \& Bloch, B. (1959). The motivation to work. New York: Wiley Press.

Hord, S. M. (1997). Professional learning communities: Communities of continuous inquiry and practice. Retrieved June 21, 2008 from http://www.sedl.org/pubs/change34/welcome.html

Huck, S. W. (2008). Reading statistics and research ( $5^{\text {th }}$ ed.). Boston, MA: Pearson Education, Inc.

Katyal, K. R., \& Evers, C. W. (2005). Teacher leadership and autonomous student learning: Adjusting to the new realities. International Journal of Educational Research, 41, 367-382.

Katzenmeyer, M., \& Moller, B. (2001). Awakening the sleeping giant: Helping teachers develop as leaders (2nd ed.). Thousand Oaks, CA: Corwin Press.

Keedy, J.L. (1999). Examining teacher instructional leadership within the small group dynamics of collegial groups. Teaching and Teacher Education, 15 (7), 785-799.

Kentucky Department of Education. (2008a). Blueprint for kentucky core content test: Weights of components of CATS system. Retrieved October 11, 2008 from http://www.kde.state.ky.us/KDE/Administrative+Resources 
/Testing+and+Reporting+CATS/Accountabilit+System/Blueprint+for+ Kentucky+Core+Content+Test.htm.

Kentucky Department of Education. (2008b). Testing and reporting: CATS.

Retrieved October 11, 2008 from http://www.kde.state.ky.us/KDE/

Administrative+Resources/Testing+and=Reporting + .

Kentucky Department of Education. (2008c). Core content for assessment.

Retrieved October 11, 2008 from www.kde.state.us/KDE/Instructional+

Resources/Curriculum+Documents+and+Resources/Core+Content+

Assessment.htm.

Kentucky Department of Education. (2008d). Elementary school. Retrieved

October 11, 2008 from www.education.ky.gov/kde/instructional+resources lelementary+school.

Kentucky Department of Education. (2008e). Kentucky's education cooperatives and special education cooperatives. Retrieved September 24, 2008 from http://education.ky.gov/kde/homepagerepository/partners+page/kentucky+ education+cooperatives.htm

Kentucky Department of Education (2008f). Kentucky core content test.

Retrieved October 11, 2008 from www.kde.state.ky.us/KDE/

Administrative+Resources/Testing+and+Reporting+Reports/Kentucky+ Core+Content+Test.htm.

Kentucky Department of Education (2008g). Kentucky performance reports.

Retrieved October 11, 2008 from http://www.kde.state.ky.us/KDE/

Administrative+Resources/Testing+and+Reporting+/Reports/Kentucky+ 
Performance+Reports.htm.

Kohler, F. W., \& Crilley, K. M. (1997). Effects of peer coaching on teacher and student outcomes. Journal of Educational Research, 90, 240-257.

Lambert, L. (1998). Building leadership capacity in schools. Alexandria, VA: Association for Supervision and Curriculum Development.

Lambert, L. (2003a). Leadership redefined: An evocative context for teacher leadership. School Leadership \& Management, 23, 421-430.

Lambert, L. (2003b). Leadership capacity for lasting school improvement. Alexandria, VA: Association for Supervision and Curriculum Development.

Lambert, L. \& Harris, A. (2003). Building leadership capacity for school improvement. Philadelphia: Open University Press.

Lapointe, M. \& Davis, S. (2006). Effective schools require effective principals. Leadership, 36, 16-38.

Lave, J. \& Wenger, E. (1991). Situated learning: Legitimate peripheral participation. Cambridge, UK: Cambridge University Press.

LeBlanc, P. R., \& Shelton, M. (1997). Teacher leadership: The needs of teachers. Action in Teacher Education, 19, 32-48.

Leithwood, K.,\& Duke, D. L. (1999). A century's quest to understand school leadership. In K. S. Louis \& J. Murphy (Eds.) Handbook of research on educational administration (2nd Ed.). San Francisco: Jossey-Bass.

Leithwood, K., \& Jantzi, D. (1999). The relative effects of principal and teacher sources of leadership on student engagement with school. Educational 
Administration Quarterly, 35, 679-706.

Leithwood, K., \& Jantzi, D. (2000). Principal and teacher leadership effects: A replication. School Leadership and Management, 20, 1363-2434.

Leithwood, K., \& Jantzi, D. (2006). Transformational school leadership for large-scale reform: Effects on students, teachers, and their classroom practices. School Effectiveness and School Improvement, 17, 201-227.

Little, J. W. (1995). Contested ground: The basis of teacher leadership in two restructuring high schools. The Elementary School Journal, 96, 47- 63.

Mangin, M. (2005). Distributed leadership and the culture of schools: Teacher leaders’ strategies for gaining access to classrooms. Journal of School Leadership, 15, 456-484.

Meyers, L.S., Gamst, G., Guarino, A.J. (2006). Applied multivariate research: Design and interpretation. Thousand Oaks, CA: Sage Publications.

Middlebrooks, G. (2004). Professionalism and teacher leadership preparation. Teacher Evaluation and Practice, 17, 432-450.

Miller, N. (2002). Insider change leadership in schools. International Journal of Leadership in Education, 5, 343-360.

Moller, G., \& Katzenmeyer, M. (1996). Every teacher as a leader: Realizing the potential of teacher leadership. San Francisco: Jossey-Bass.

Muijs, D., \& Harris, A. (2003). Teacher leadership: Improvement through empowerment. Educational Management \& Administration, 31, 437-448.

Muijs, D. \& Harris, A. (2006). Teacher-lead school improvement: Teacher leadership in the UK. Teaching and Teacher Education, 22, 961-972. 
Murphy, J. (2005). Connecting teacher leadership and school improvement. Thousand Oaks, CA: Corwin Press.

National Commission on Excellence in Education. (1983, April), A nation at risk: The imperative for educational reform. Retrieved October 30, 2006, from http://www.ed.gov/pubs/NatAtRisk/risk

National Staff Development Council. (2001a). Learning communities. Retrieved June 21, 2008 from www.nsdc.org/standards/learningcommunities

National Staff Development Council. (2001b). NSDC's standards for staff development. Retrieved June 21, 2008 from www.NSDC.org

Nutrition Health Services. (2008). \% Free/reduced lunch students in GRREC elementary Schools for 2007-2008. Retrieved February 2, 2009 from http://nhs.ky.gov/octdataout/rptlist.htm

Ovando, M. (1996). Teacher leadership: Opportunities and challenges. Planning and Changing, 27, 30-44.

Pedhazur, E. J. (1997). Multiple regression in behavioral research: Explanation and prediction. Canada: Thomson Learning, Inc.

Pellicer, L.O., \& Anderson, L.W. (1995). A handbook for teacher leaders. Thousand Oaks, CA: Corwin Press.

Sawyer, R. D. (2001). Teachers who grow as collaborative leaders: The rocky road of support. Educational Policy Analysis Archives, 9 (38). Retrieved January 21, 2007 from http://epaa.asu.edu/epaa/v9n38.html.

Schmoker, M.J., \& Wilson, R.B. (1994). Redefining results: Implications for teacher leadership and professionalism. In D.R. Walling (Ed.), Teachers 
as leaders: Perspectives on the professional development of teachers. Bloomington, IN: Phi Delta Kappa

Shavelson, R.J. (1988). Statistical reasoning for the behavioral sciences $\left(2^{\text {nd }}\right.$ Ed.) Boston: Allyn and Bacon, Inc.

Silins, H., \& Mulford, B. (2004). Schools as learning organizations: Effects on teacher leadership and student outcomes. School Effectiveness and School Improvement, 15, 443-466.

Silva, D., Gimbert, B., \& Nolan, J. (2000). Sliding the doors: Locking and unlocking possibilities for teacher leadership. Teachers College Record, 102, 779-804.

Smylie, M. (1992a). Teacher participation in school decision making: Assessing willingness to participate. Educational Evaluation and Policy Analysis, $14,53-67$.

Smylie, M. (1992b). Teachers' reports of their interactions with teacher leaders concerning classroom instruction. The Elementary School Journal, 93(1), 85-98.

Smylie, M. (1996). Research on teacher leadership: Assessing the state of the art. In B. J. Biddle, T.L. Good, \& I.F. Goodson. (Eds.), International handbook of teachers and teaching (pp. 551-592). Dordrecht, The Netherlands: Kluwer Academic Publishers.

Smylie, M. A., \& Brownlee-Conyers, J. (1992). Teacher leaders and their principals: Exploring the development of new working relationships. Educational Administration Quarterly, 28, 150-184. 
Smylie, M., \& Denny, J. (1990). Teacher leadership: Tensions and ambiguities in organizational perspective. Educational Administration Quarterly, 26, 235-259.

Smylie, M.A., \& Hart, A.W. (1999). School leadership for teacher learning: A human and social capital development perspective. In J. Murphy \& K.S. Louis, Handbook of research on educational administration $\left(2^{\text {nd }}\right.$ ed., pp. 421441). San Francisco: Jossey-Bass.

Smylie, M., Lazarus, V., \& Brownlee-Conyers, J. (1996). Instructional outcomes of school-based participative decision making. Educational Evaluation and Policy Analysis, 18, 181-198.

Spillane, J. P., Hallett, T., \& Diamond, J. B. (2003). Forms of capital and the construction of leadership: Instructional leadership in urban elementary schools. Sociology in Education, 76, 1-17.

Spillane, J. P., Halverson, R., \& Diamond, J. B. (2004). Towards a theory of leadership practice: A distributed perspective. Journal of Curriculum Studies, 36, 3-34.

Stone, M., Horejs, J., \& Iomas, A. (1997). Commonalities and differences in teacher leadership at the elementary, middle, and high school levels. Action in Teacher Education, 19, 49-64.

Superintendent Average Attendance Report. (2008). SAAR. Retrieved February 2, 2009 From http://www.education.ky.gov/KDE?Administrative+Resources/Finance + and+Funding/School+Finance/Attendance+Data/SAAR+Ethnic+Membership +Report.htm 
Taylor, D. L., \& Bogotch, I. E. (1994). School-level effects of teacher's participation in decision making. Educational Evaluation and Policy Analysis, 16, 302-319.

Temperley, H. S. (2005). Distributed leadership: Developing theory from practice. Journal of Curriculum Studies, 37, 395-420.

Thompson, S., Gregg, L., \& Niska, J. (2004). Professional learning communities, leadership, and student learning. Research in Middle Level Education Online, 28, 1-16.

Turnbull, B., \& Mee, J. (2003). Teacher perspectives of school management teams. Educational Research and Evaluation, 9, 373-389.

Vail, A. \& Redick, S. (1993). Predictors of teacher leadership performance of vocational and nonvocational teachers. Journal of Vocational Education Research, 18, 51-76.

Vogt, W. P. (2005). Dictionary of statistics and methodology: A nontechnical guide for the social sciences $\left(2^{\text {nd }}\right.$ ed.). Thousand Oaks, CA: Sage Publications.

Wasley, P. A. (1991). Teachers who lead: The rhetoric of reform and the realities of practice. New York: Teachers College Press.

Waters, T., Marzano, R. J., \& McNulty, B. (2003). Balanced leadership: What 30 years of research tells us about the effect of leadership on student achievement. A working paper. Aurora, CO: Mid-Continent Research for Education and Learning

Wenglinsky, H. (2002). How schools matter: The link between teacher classroom practices and student academic performance. Education Policy Analysis 
Archives, 10(12). Retrieved January 21, 2007 from

http://epaa.asu.edu/epaa/v10n12/.

Whitaker, K. S. (1997). Developing teacher leadership and the management.team concept: A case study. Teacher Education, 33, 1-16.

White, P. (1992). Teacher empowerment under "ideal" school-site autonomy. Educational Evaluation and Policy Analysis, 14, 69 - 82.

Wunsch, D.R. (1986). Survey research: Determining sample size and representative response. Business Education Forum, 40, 31-34.

Yarger, S. J., \& Lee, O. (1994). The development and sustenance of instructional leadership. In D.R. Walling (Ed.), Teachers as leaders: Perspectives on the professional development of teachers. Bloomington, IN: Phi Delta Kappa.

York-Barr, J., \& Duke, K. (2004). What do we know about teacher leadership? Findings from two decades of scholarship. Review of Educational Research, 74, 255-316. 


\section{APPENDIX A}

\section{CONTENT VALIDITY SURVEY}

The content validity survey below includes a Likert-like scale rating for each item. Please circle one number for each item to indicate your opinion of its content appropriateness for the construct explained above each item set.

The number 5 is used to indicate a very strong item, and the number 1 is used to indicate a very poor item. If you wish, you may write comments next to or near any item.

Please rate each set of items for their appropriateness in measuring the construct regarding the types of interactions an identified teacher leader (i.e., curriculum specialist, curriculum leader, etc.) would have with teachers in a school.

A. Broad-based, skillful participation in the work of leadership. This means that school organizational structures support opportunities for teachers and teacher leaders to collaborate on meaningful tasks. Occasions for observing, practicing, and receiving coaching on key collaborative skills, such as facilitating, communicating, and mediating are available.

1. Assists in setting up committees and work groups within the school.

2. Organizes to maximum interaction among all school and community members

3. Shares authority and resources broadly.

4. Engages others in opportunities to lead

5. Models, describes, and demonstrates the following leadership skills:

a. develops common understanding of school-wide learning goals

b. facilitates group work

c. communicates (listens, questions)

$\begin{array}{lllll}1 & 2 & 3 & 4 & 5\end{array}$

d. promotes reflection on instructional practice

$\begin{array}{lllll}1 & 2 & 3 & 4 & 5\end{array}$ 
e. seeks answers to school-wide questions/issues

f. facilitates collaboration

g. manages conflict among adults

h. facilitates problem-solving with teachers and students

i. helps teachers deal with change and difficult transitions

j. facilitates analysis of student work against standards

k. facilitates development of learning opportunities that connect content with real-world experiences

6. Communicates with words and actions the connection between leadership and learning $\begin{array}{lllll}1 & 2 & 3 & 4 & 5\end{array}$

$\begin{array}{lllll}1 & 2 & 3 & 4 & 5\end{array}$

$\begin{array}{lllll}1 & 2 & 3 & 4 & 5\end{array}$

$\begin{array}{lllll}1 & 2 & 3 & 4 & 5\end{array}$

$\begin{array}{lllll}1 & 2 & 3 & 4 & 5\end{array}$

$\begin{array}{lllll}1 & 2 & 3 & 4 & 5\end{array}$

$\begin{array}{lllll}1 & 2 & 3 & 4 & 5\end{array}$

$\begin{array}{lllll}1 & 2 & 3 & 4 & 5\end{array}$

\section{Content Validity Notes:}


B. Inquiry-based use of information to inform shared decisions and practice. This means that an inquiry-based use of information shapes school decisions and instructional practice. Reflection, conversation, posing of questions in response to data are regular interactions between teachers and teacher leaders.

7. Works with teachers in a learning cycle (reflection, dialogue, question-posing, inquiry, construction of meaning, planned action).

8. Develops plans and schedules for teachers to collaborate and reflect.

9. Identifies, discovers, and interprets data regarding student achievement.

10. Designs and implements a communication system that keeps everyone informed about gathering and interpreting data.

11. Participates with teachers to integrate student achievement data into decision making.

\section{Content Validity Notes:}


C. Roles and responsibilities that reflect broad involvement and collaboration among teachers. This points toward changes in teachers' perceptions about themselves and their role in the work of the school as a result of on-going interactions with teacher leaders and colleagues. These changed perceptions can lead to changed relationships and instructional practices.

12. Pays attention to classroom teacher concerns and school-wide, community, and professional issues.

13. Works at building trusting, supportive relationships.

14. Provides meaningful feedback to teachers about planning, instruction, and student achievement.

$\begin{array}{lllll}1 & 2 & 3 & 4 & 5\end{array}$

15. Develops expectations and strategies to ensure that all stakeholders share responsibility for implementation of the school vision.

$\begin{array}{lllll}1 & 2 & 3 & 4 & 5\end{array}$

16. Develops strategies to encourage teachers to attempt non-traditional teacher roles.

\section{Content Validity Notes:}


D. Reflective practice and innovation are the norm. This indicates that conversations between teachers and teacher leaders lead to reflection about beliefs, assumptions, content standards, learning styles, and instructional practice are commonplace and non-threatening. Collaborative planning, monitoring and evaluating, innovative and risk-taking are encouraged through regular interaction.

17. Supports teachers who are innovative and take risks to improve student learning.

18. Ensures continuous and ongoing reflection on teacher work.

19. Demonstrates and encourages individual and group initiative by providing access to resources, personnel, time, and outside networks.

20. Encourages and participates in collaborative innovation. $\begin{array}{lllll}1 & 2 & 3 & 4 & 5\end{array}$

21. Works with others to develop criteria for monitoring and assessing implementation of school-wide programs, as well as individual and shared accountability for student learning.

\section{Content Validity Notes:}


E. Analysis of Learning. This means that students successfully perform authentic tasks, i.e. tasks that have a real-world relationship to work or the general society. Achievement data comes from multiple types and forms of assessment at various points in time and is used as evidence to determine who needs specific interventions to master the content, and who needs deeper learning opportunities.

22. Works with others to develop criteria for monitoring and assessing implementation of school-wide programs, as well as individual and shared accountability for student learning.

23. Works with others to establish rigorous expectations for all students.

$\begin{array}{lllll}1 & 2 & 3 & 4 & 5\end{array}$

24. Designs, teachers, coaches, and assess curriculum, instruction, and assessment processes which ensure that all children learn.

25. Provides regular, meaningful, feedback to students and parents about student progress.

$\begin{array}{lllll}1 & 2 & 3 & 4 & 5\end{array}$

26. Provides meaningful feedback to colleagues.

$\begin{array}{lllll}1 & 2 & 3 & 4 & 5\end{array}$

27. Accepts feedback from students and parents.

$\begin{array}{lllll}1 & 2 & 3 & 4 & 5\end{array}$

28. Accepts feedback from colleagues.

$\begin{array}{lllll}1 & 2 & 3 & 4 & 5\end{array}$

29. Re-designs roles and structures to help the school develop and sustain resiliency in children (e.g. teacher as coach/mentor).

30. Ensures that evidence of learning includes assessment data, analysis of student work, and research-based instructional strategies.

\section{Content Validity Notes:}




\title{
APPENDIX B
}

\author{
Survey Instrument
}

TEACHER LEADER INTERACTIONS WITH TEACHERS SURVEY

\author{
$\underline{\text { Preamble }}$
}

December 1, 2008

Dear Educator:

You are invited to participate in a research study by completing the attached survey about the interactions between teachers and teacher leaders. This study is being conducted by Winifred R. Cohron, an Instructional Supervisor in a Kentucky school district and a student in the Western Kentucky (WKU) University/University of Louisville (UofL) Cooperative Doctoral Program, and Dr. Jeanne Fiene of Western Kentucky University. This research is sponsored by the Department of Educational Administration, Leadership and Research at Western Kentucky University, and the Department of Leadership, Foundations, and Human Resource Education at the University of Louisville.

Participation in this study is voluntary. By completing this survey, you agree to take part in this research study. The survey should take approximately 15 minutes to complete. There are no known risks for your participation in this research study. The information collected may not benefit you directly, but it may help Kentucky schools learn how to more effectively utilize teacher leader personnel.

Your completed survey will be stored at Western Kentucky University within the Department of Educational Administration, Leadership and Research. Individuals from the Department of Educational Administration, Leadership and Research at Western Kentucky University, the Department of Leadership, Foundations, and Human Resource Education of the University of Louisville, and the University of Louisville Human Subjects Protection Program Office (HSPPO), and the Human Subjects Review Board (HSRB) at Western Kentucky University, as well as other regulatory agencies may inspect these records. In all other respects, however, the data will be held in confidence to the extent permitted by law. Should the data be published, your identity will not be disclosed.

You do not have to answer any questions that make you uncomfortable. You may choose not to take part at all. If you decide to be in this study you may stop taking part at any 
INSTRUCTIONS: The items below describe a variety of ways that teachers and teacher leaders may interact. Circle the one number for each item that best represents how the identified teacher leader (curriculum coordinator, curriculum specialist, etc.) in your school interacted with you during the 2007-2008 academic year.

If your school does not employ an identified teacher leader (curriculum coordinator, curriculum specialist, etc.), please circle the number of each item that best represents how a teacher who exercises informal leadership in your school (i.e., teaching partner, gradelevel colleague, leadership team member) interacted with you during the 2007-2008 academic year.

Use the following scale:

$$
\begin{aligned}
& 1=\text { Never Performed } \\
& 2=\text { Infrequently Performed } \\
& 3=\text { Frequently Performed } \\
& 4=\text { Consistently Performed } \\
& 5=\text { An Established Behavior }
\end{aligned}
$$

\section{STATEMENT}

1. Assists in setting up committees and work groups within the school.

2. Organizes to maximize interactions among all school and community members.

3. Shares resources broadly.

4. Engages teachers in opportunities to lead.

5. Develops common understanding among teachers of school-wide learning goals.

6. Facilitates collaborative group work among teachers.

7. Facilitates communication by listening, questioning, and writing.

8. Promotes reflection on instructional practice.

9. Promotes exploration and research about school-wide teaching and learning issues.

\section{FREQUENCY}

$\begin{array}{lllll}1 & 2 & 3 & 4 & 5\end{array}$

$\begin{array}{lllll}1 & 2 & 3 & 4 & 5\end{array}$

$\begin{array}{lllll}1 & 2 & 3 & 4 & 5\end{array}$

$\begin{array}{lllll}1 & 2 & 3 & 4 & 5\end{array}$

$\begin{array}{lllll}1 & 2 & 3 & 4 & 5\end{array}$

$\begin{array}{lllll}1 & 2 & 3 & 4 & 5\end{array}$

$\begin{array}{lllll}1 & 2 & 3 & 4 & 5\end{array}$

$\begin{array}{lllll}1 & 2 & 3 & 4 & 5\end{array}$

$\begin{array}{lllll}1 & 2 & 3 & 4 & 5\end{array}$ 
$1=$ Never Performed

$2=$ Infrequently Performed

$3=$ Frequently Performed
$4=$ Consistently Performed

5 = An Established Behavior
10. Manages conflict among adults.

11. Facilitates problem solving with teachers.

12. Helps teachers deal with change and difficult transitions.

13. Facilitates analysis of student work based on content standards.

14. Facilitates development of learning opportunities that connect content with real-world experiences.

15. Communicates with words and actions the connection between leadership and learning.

16. Works with teachers to develop standards-based classroom instruction responsive to student learning needs.

17. Develops plans and schedules for teacher collaboration and reflection.

18. Identifies and interprets student achievement data.

19. Designs and implements a system to gather and interpret student achievement data.

20. Participates with teachers in using student achievement data to inform instructional decisions.

21. Pays attention to classroom teacher concern related to school and professional issues.

22. Provides meaningful feedback about planning for instruction.

23. Provides meaningful feedback about classroom Instruction. $\begin{array}{lllll}1 & 2 & 3 & 4 & 5\end{array}$

$\begin{array}{lllll}1 & 2 & 3 & 4 & 5\end{array}$

$\begin{array}{lllll}1 & 2 & 3 & 4 & 5\end{array}$

$\begin{array}{lllll}1 & 2 & 3 & 4 & 5\end{array}$

$\begin{array}{lllll}1 & 2 & 3 & 4 & 5\end{array}$

$\begin{array}{lllll}1 & 2 & 3 & 4 & 5\end{array}$

$\begin{array}{lllll}1 & 2 & 3 & 4 & 5\end{array}$

$\begin{array}{lllll}1 & 2 & 3 & 4 & 5\end{array}$

$\begin{array}{lllll}1 & 2 & 3 & 4 & 5\end{array}$

$\begin{array}{lllll}1 & 2 & 3 & 4 & 5\end{array}$

$\begin{array}{lllll}1 & 2 & 3 & 4 & 5\end{array}$

$\begin{array}{lllll}1 & 2 & 3 & 4 & 5\end{array}$

$\begin{array}{lllll}1 & 2 & 3 & 4 & 5\end{array}$

$\begin{array}{lllll}1 & 2 & 3 & 4 & 5\end{array}$ 


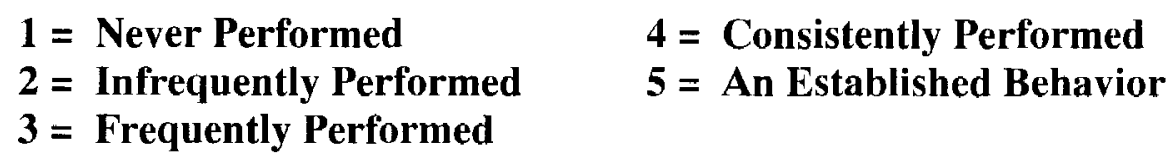

24. Provides meaningful feedback about student achievement.

$$
\begin{array}{lllll}
1 & 2 & 3 & 4 & 5
\end{array}
$$

25. Builds trusting, supportive relationships with teachers. $\begin{array}{llllll} & 1 & 2 & 3 & 4 & 5\end{array}$

26. Supports teachers who are innovative and who take risks to improve student learning.

$\begin{array}{lllll}1 & 2 & 3 & 4 & 5\end{array}$

27. Encourages teachers to attempt non-traditional teacher roles.

28. Stimulates shared responsibility for implementation of the school vision.

29. Supports continuous and ongoing reflection by teachers on teacher work.

$\begin{array}{lllll}1 & 2 & 3 & 4 & 5\end{array}$

30. Provides access to resources, personnel, time, and outside networks.

31. Practices and supports innovation without expectation of instant success.

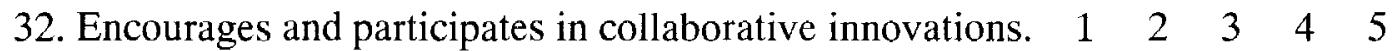

33. Works with others to develop criteria to monitor and assess implementation of school-wide programs.

34. Works with others to develop criteria for individual and shared accountability for student learning.

35. Works with others to establish rigorous expectations for all students.

36. Designs, teaches, coaches, and evaluates curriculum.

$\begin{array}{lllll}1 & 2 & 3 & 4 & 5\end{array}$

37. Designs, teaches, coaches, and evaluates instruction.

$\begin{array}{lllll}1 & 2 & 3 & 4 & 5\end{array}$

38. Designs, teaches, coaches and evaluates assessment.

$\begin{array}{lllll}1 & 2 & 3 & 4 & 5\end{array}$



time. If you decide not to be in this study or if you stop taking part at any time, you will not lose any benefits for which you may qualify.

If you have any questions, concerns, or complaints about the research study, please contact the principal researcher: Dr. Jeanne Fiene, at (270) 745-2942. If you have any questions about your rights as a research subject, you may call the UofL Human Subjects Protection Program Office at (502) 852-5188 or the WKU Human Subjects Review Board (HSRB) at (270) 745-4652. You can discuss any questions about your rights as a research subject, in private, with a member of the Institutional Review Board (IRB) or the WKU Human Subjects Review Board (HSRB). You may also call this number if you have other questions about the research, and you cannot reach the research staff, or want to talk to someone else. The UofL IRB and the WKU HSRB are independent committees made up of people from the University community, staff of the institutions, as well as people from the community not connected with these institutions. The UofL IRB and the WKU HSRB have reviewed this research study.

If you have concerns or complaints about the research or research staff and you do not wish to give your name, you may call 1-877-852-1167. This is a 24 hour hot line answered by people who do not work at Western Kentucky University or the University of Louisville.

Sincerely,

Jeanne Fiene, Ph.D.

Winifred R. Cohron

Please continue to the next page. 
$1=$ Never Performed

2 = Infrequently Performed

$3=$ Frequently Performed
$4=$ Consistently Performed

$5=$ An Established Behavior

39. Implements a system for regular, meaningful feedback to students and parents about student progress.

$\begin{array}{lllll}1 & 2 & 3 & 4 & 5\end{array}$

40. Provides meaningful feedback to colleagues.

$\begin{array}{lllll}1 & 2 & 3 & 4 & 5\end{array}$

41. Accepts feedback from students and parents and amends practice when appropriate.

$\begin{array}{lllll}1 & 2 & 3 & 4 & 5\end{array}$

42. Accepts feedback from colleagues and amends practice when appropriate.

$\begin{array}{lllll}1 & 2 & 3 & 4 & 5\end{array}$

43. Ensures that evidence of learning includes assessment data and analysis of student work.

$\begin{array}{lllll}1 & 2 & 3 & 4 & 5\end{array}$

Thank you for participating in this survey. 


\section{APPENDIX C}

Test/Re-test Correlations

\begin{tabular}{cc}
\hline Item & $\begin{array}{c}\text { Pearson } \\
\text { Correlation }\end{array}$ \\
\hline
\end{tabular}

1. Assists in setting up committees and work groups within the school.

2. Organizes for maximum interactions among all school and community $\quad .557$ members.

3. Shares authority and resources broadly.

4. Engages others in opportunities to lead. $\quad .712$

5. Models, describes, and demonstrates the following leadership skills:

a. develops common understanding of school-wide learning goals. $\quad .733$

b. facilitates group work. $\quad .897$

$\begin{array}{ll}\text { c. communicates (listens, questions). } & .800\end{array}$

d. promotes reflection on instructional practice. $\quad .585$

e. seeks answers to school-wide questions / issues.

f. facilitates collaboration. $\quad .730$

$\begin{array}{ll}\text { g. manages conflict among adults. } & .787\end{array}$

h. facilitates problem solving with teachers and students.

$\begin{array}{ll}\text { i. helps teachers deal with change and difficult transitions. } & 567\end{array}$

j. facilitates analysis of student work against standards. $\quad .836$

k. facilitates development of learning opportunities that connect content $\quad .881$ with real-world experience. 


\begin{tabular}{cc}
\hline Item & $\begin{array}{c}\text { Pearson } \\
\text { Correlation }\end{array}$ \\
\hline
\end{tabular}

6. Communicates with words and actions the connection between .683 leadership and learning.

7. Works with teachers in a learning cycle (reflection, dialogue, questionposing, inquiry, construction of meaning, planned action).

8. Develops plans and schedules for teachers to collaborate and reflect.

9. Identifies, discovers, and interprets data regarding student achievement.

10. Designs and implements a communication system that keeps everyone informed about gathering and interpreting data.

11. Participates with teachers to integrate student achievement data into decision making

12. Pays attention to classroom teacher concerns and school-wide, community, and professional issues.

13. Provides meaningful feedback to teachers about planning, instruction, and student achievement.

14. Works at building trusting, supportive relationships.

15. Supports teachers who are innovative and take risks to improve student learning.

16. Develops strategies to encourage teachers to attempt non-traditional teacher roles.

17. Develops expectations and strategies to ensure that all stakeholders share responsibility for implementation of the school vision.

18. Ensures continuous and ongoing reflection on teacher work.

19. Demonstrates and encourages individual and group initiative by providing access to resources, personnel, time, and outside networks.

20. Practices and supports innovation without expectations for instant success.

21. Encourages and participates in collaborative innovation. 
22. Works with others to develop criteria for monitoring and assessing implementation of school-wide programs, as well as individual and shared accountability for student learning.

23. Works with others to establish rigorous expectations for all students.

24. Designs, teaches, coaches, and assesses curriculum, instruction, and assessment processes which ensure all children learn.

25. Provides regular, meaningful feedback to students and parents about student progress.

26. Provides meaningful feedback to colleagues.

27. Accepts feedback from students and parents.

28. Accepts feedback from colleagues

29. Re-designs roles and structures to help the school develop and sustain resiliency in children (e.g., teacher as coach, mentor).

30. Ensures that evidence of learning includes assessment data, analysis of student work, and research-based instructional strategies.

Leadership Work Construct (Questions 1-6)

Shared Decisions Construct (Questions 7-11)

Collaboration Construct (Questions 12-17)

Reflective Practice Construct (Questions 18-21) 


\section{APPENDIX D}

\section{Superintendent Permission Form}

Study:

The Effect of Teacher Leader Interactions With Teachers on Student Achievement: A Predictive Study

Researchers: $\quad$ Jeanne Fiene, Ph.D., WKU, Principal Researcher

Winifred R. Cohron, Doctoral Candidate, UofL/WKU Cooperative Doctoral Program

Desired

Elementary teachers in the GRREC service region

Participants:

Method: $\quad$ Survey adapted from Dr. Linda Lambert's Leadership

$$
\text { Capacity Staff Survey (1998) with permission }
$$

Time: $\quad$ Early to mid-February

I give permission for the elementary teachers in my school district to be invited to participate in the survey for the above described research study.

School District Name (please print)

$\overline{\text { Superintendent's Name (please print) }}$

Superintendent's Signature

Date

E-mail: Winifred.cohron(awarren.kyschools.us

FAX: 270-781-2392

Mail: P.O. Box 51810

Bowling Green, KY 42103 


\title{
APPENDIX E
}

\author{
Principal E-mail
}

Dear Principal,

This e-mail requesting your assistance with gathering data for my doctoral dissertation research is sent with the permission of your school district superintendent. The research deals with the relationship between specific school-level teacher leader interactions with teachers and student achievement.

With your assistance and that of the faculty members of your school, it is hoped that sufficient data can be gathered to see if specific interactions more readily predict student achievement.

School districts in the Green River Regional Education Cooperative (GRREC) have been invited to participate in this study. It is my sincere hope that teachers in your school will participate by responding to the survey questions at this website:

http://www.wku.edu/phpESP/public/survey.php?name=Teacher Leader Inter actions With Teachers

Please forward this e-mail to all the faculty members in your school and ask them to respond within seven (7) days. Your help in this initiative is greatly appreciated.

Sincerely,

Winnie Cohron

Instructional Supervisor

Warren County Public Schools

UofL/WKU Doctoral Candidate

270.781 .5150

Winifred.cohron@warren.kyschools.us 


\section{APPENDIX F}

\section{Expanded School Participation Data}

\begin{tabular}{|c|c|c|c|}
\hline School & Population & Frequency & $\begin{array}{l}\text { Valıd } \\
\text { Percent }\end{array}$ \\
\hline Adair County Elementary & 42 & 5 & 0.9 \\
\hline Albany Elementary School & 42 & 6 & 1.1 \\
\hline Allen County Intermediate Center & 39 & 12 & 2.2 \\
\hline Allen County Primary Center & 61 & 19 & 2.7 \\
\hline Alvaton Elementary School & 29 & 12 & 2.2 \\
\hline Audubon Elementary School & 34 & 2 & 0.4 \\
\hline Battletown Elementary School & 21 & 2 & 0.4 \\
\hline Briarwood Elementary School & 40 & 6 & 1.1 \\
\hline Bristow Elementary School & 39 & 16 & 2.9 \\
\hline Burns Elementary School & 41 & 6 & 1.1 \\
\hline Caneyville Elementary School & 31 & 5 & 0.9 \\
\hline Clarkson Elementary School & 41 & 7 & 1.3 \\
\hline Colonel William Casey Elementary & 31 & 3 & 0.5 \\
\hline Country Heights Elementary School & 26 & 8 & 1.4 \\
\hline Cumberland County Elementary & 36 & 1 & 0 . \\
\hline Cumberland Trace Elementary School & 28 & 18 & 3.2 \\
\hline David T. Wilson Elementary & 25 & 5 & \\
\hline
\end{tabular}




\begin{tabular}{|c|c|c|c|}
\hline School & Population & Frequency & $\begin{array}{r}\text { Valid } \\
\text { Percent }\end{array}$ \\
\hline Deer Park Elementary School & 30 & 3 & 0.5 \\
\hline Dishman McGinnis Elementary & 18 & 12 & 2.2 \\
\hline East View Elementary School & 33 & 9 & 1.6 \\
\hline Edmonson Co 5-6 Center & 19 & 3 & 0.5 \\
\hline Edmonton Elementary School & 34 & 6 & 1.1 \\
\hline Ekron Elementary School & 26 & 1 & 0.2 \\
\hline Estes Elementary School & 31 & 2 & 0.4 \\
\hline Gamaliel Elementary School & 21 & 4 & 0.7 \\
\hline Greensburg Elementary School & 35 & 9 & 1.6 \\
\hline H W Wilkey Elementary School & 36 & 9 & 1.6 \\
\hline Highland Elementary & 38 & 30 & 2 \\
\hline Highland Elementary School & 34 & 11 & 5.4 \\
\hline Joe Harrison Carter Elementary & 23 & 13 & 2.3 \\
\hline John Adair Intermediate & 26 & 3 & 0.5 \\
\hline Kyrock Elementary School & 23 & 4 & 0.7 \\
\hline Lincoln Elementary School & 23 & 11 & 2 \\
\hline Lost River Elementary School & 46 & 23 & 4.1 \\
\hline Meadow Lands Elementary School & 27 & 6 & 1.1 \\
\hline Newton Parrish Elementary School & 31 & 5 & 0.9 \\
\hline North Metcalfe Elementary School & 9 & 1 & 0.2 \\
\hline North Todd Elementary School & 38 & 7 & 1.3 \\
\hline North Warren Elementary School & 27 & 17 & 3.1 \\
\hline Oakland Elementary School & 20 & 7 & 1.3 \\
\hline
\end{tabular}




\begin{tabular}{|c|c|c|c|}
\hline School & Population & Frequency & $\begin{array}{r}\text { Valid } \\
\text { Percent }\end{array}$ \\
\hline Oran P Lawler Elementary School & 30 & 2 & 0.4 \\
\hline Owensboro 5-6 Elementary Center & 37 & 4 & 0.7 \\
\hline Parker Bennett Curry Elementary & 23 & 11 & 2 \\
\hline Payneville Elementary School & 9 & 8 & 1.4 \\
\hline Pierce Elementary School & 8 & 1 & 0.2 \\
\hline Plano Elementary & 27 & 18 & 3.2 \\
\hline Potter Gray Elementary & 23 & 1 & 0.2 \\
\hline R E Stevenson Elementary School & 39 & 16 & 2.9 \\
\hline Rich Pond Elementary School & 38 & 17 & 3.1 \\
\hline Richardsville Elementary School & 28 & 13 & 2.3 \\
\hline Rockfield Elementary School & 33 & 10 & 1.8 \\
\hline Simpson Elementary School & 42 & 4 & 0.7 \\
\hline Sorgho Elementary School & 32 & 3 & 0.5 \\
\hline South Edmonson Elementary School & 32 & 1 & 0.2 \\
\hline South Green Elementary School & 33 & 19 & 3.4 \\
\hline South Hancock Elementary School & 16 & 2 & 0.4 \\
\hline South Todd Elementary School & 38 & 1 & 0.2 \\
\hline Sturgis Elementary & 26 & 2 & 0.4 \\
\hline Summer Shade Elementary School & 11 & 1 & 0.2 \\
\hline T C Cherry Elementary & 17 & 10 & 1.8 \\
\hline Tamarack Elementary School & 30 & 9 & 1.6 \\
\hline Taylor County Elementary School & 76 & 4 & 0.7 \\
\hline Tompkinsville Elementary School & 33 & 15 & 2.7 \\
\hline Uniontown Elementary & 14 & 8 & 1.4 \\
\hline
\end{tabular}


School

Utica Elementary

W R McNeill Elementary

Warren County Elementary School

West Louisville Elementary School

Whitesville Elementary School

William Natcher Elementary School

$\begin{array}{rrr}\text { Population } & \text { Frequency } & \begin{array}{r}\text { Valid } \\ \text { Percent } \\ 14\end{array} \\ 20 & 16 & 1.4 \\ 43 & 8 & 2.9 \\ 21 & 2 & 0.4 \\ 25 & 4 & 0.7 \\ 43 & 14 & 2.5 \\ \mathbf{2 1 1 5} & \mathbf{5 6 1} & \mathbf{1 0 0 . 4}\end{array}$




\section{APPENDIX G}

District Participation Data

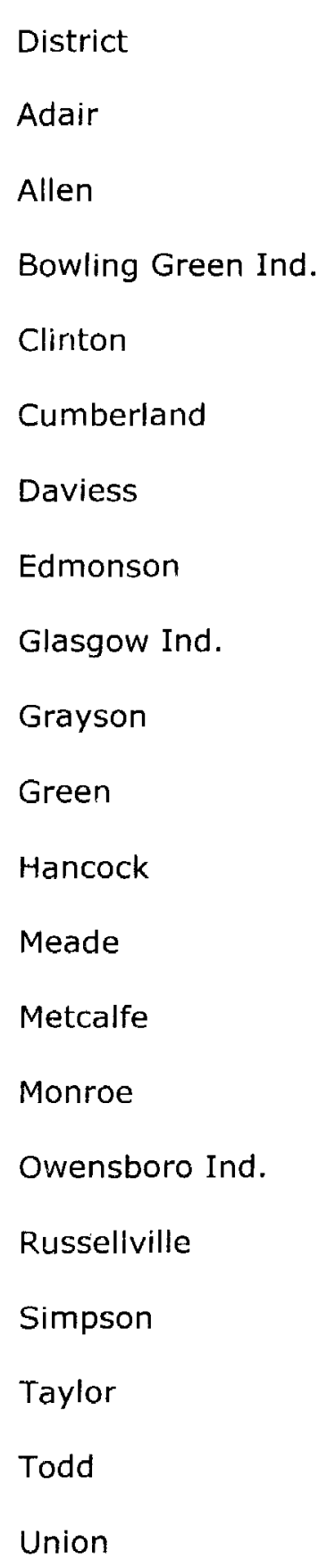

\begin{tabular}{|c|c|c|}
\hline Population & Frequency & $\begin{array}{r}\text { Valid } \\
\text { Percent }\end{array}$ \\
\hline 99 & 11 & 1.9 \\
\hline 100 & 31 & 4.9 \\
\hline 101 & 50 & 9.1 \\
\hline 42 & 6 & 1.1 \\
\hline 36 & 1 & 0.2 \\
\hline 347 & 71 & 16.1 \\
\hline 74 & 8 & 1.4 \\
\hline 71 & 49 & 5.4 \\
\hline 138 & 23 & 4.2 \\
\hline 43 & 10 & 1.8 \\
\hline 16 & 2 & 0.4 \\
\hline 81 & 16 & 2.9 \\
\hline 54 & 8 & 1.5 \\
\hline 77 & 32 & 5.7 \\
\hline 99 & 11 & 2 \\
\hline 39 & 16 & 2.9 \\
\hline 65 & 15 & 2.7 \\
\hline 76 & 4 & 0.7 \\
\hline 76 & 8 & 1.5 \\
\hline 40 & 10 & 1.8 \\
\hline
\end{tabular}


District

$\begin{array}{rrr}\text { Population } & \text { Frequency } & \text { Valid } \\ 441 & 179 & 32.2\end{array}$

Warren

179

2115

561

100.4 


\section{APPENDIX H}

2008 Academic Indices for Participating Schools

District

School

2008

Academic

Index

Adair

Adair County Elementary

81.2

Adair

Colonel William Casey Elementary

93.7

Adair

John Adair Intermediate

93.7

Allen

Allen County Intermediate Center

88.7

Allen County Primary Center

99

Dishman McGinnis Elementary

76.2

Parker Bennett Curry Elementary

78

Potter Gray Elementary

111.7

T.C Cherry Elementary

92

W R McNeill Elementary

111.2

Albany Elementary School

87.2

Cumberland County Elementary

82.9

Audubon Elementary School

105

Burns Elementary School

93.7

Country Heights Elementary School

99.5

Deer Park Elementary School

108.1

East View Elementary School

101.7

Highland Elementary School

113

Meadow Lands Elementary School

87.6

Sorgho Elementary School

110.2

Tamarack Elementary School

100.4 
Daviess

West Louisville Elementary School

107.4

Daviess

Whitesville Elementary School

104.6

25 Edmonson

Edmonson Co 5-6 Center

91.4

26 Edmonson

Kyrock Elementary School

91.4

Edmonson

Glasgow

South Edmonson Elementary School

91.4

South Green Elementary School

106.1

29 Glasgow Inde

Highland Elementary

99

30

Grayson

Caneyville Elementary School

90.6

Clarkson Elementary School

93.6

H W Wilkey Elementary School

88

33

Grayson

Oran P Lawler Elementary School

93.3

Greensburg Elementary School

90.7

Green

Green

Pierce Elementary School

100.3

Hancock

South Hancock Elementary School

97.9

37

Meade

38

39

40

41

Meade

Battletown Elementary School

85.5

David T. Wilson Elementary

101.1

Ekron Elementary School

90.5

Payneville Elementary School

101

Edmonton Elementary School

84

North Metcalfe Elementary School

99.5

Summer Shade Elementary School

98.1

Metcalfe

Gamaliel Elementary School

101.4

45

Monroe

Joe Harrison Carter Elementary School

100.7

46 Monroe

Tompkinsville Elementary School

104.6

47 Owensboro

Estes Elementary School

102

48 Owensboro

Newton Parrish Elementary School

102

Owensboro 5-6 Elementary Center 102

49 Owensboro

R E Stevenson Elementary School

81.9

51

Simpson

Lincoln Elementary School

93.8 


$\begin{array}{rccr}52 & \text { Simpson } & \text { Simpson Elementary School } & 93.8 \\ 53 & \text { Taylor } & \text { Taylor County Elementary School } & 89.5 \\ 54 & \text { Todd } & \text { North Todd Elementary School } & 94.8 \\ 55 & \text { Todd } & \text { South Todd Elementary School } & 90.5 \\ 56 & \text { Union } & \text { Sturgis Elementary } & 92.4 \\ 57 & \text { Union } & \text { Uniontown Elementary } & 96.7 \\ 58 & \text { Warren } & \text { Alvaton Elementary School } & 91.2 \\ 59 & \text { Warren } & \text { Briarwood Elementary School } & 101.7 \\ 60 & \text { Warren } & \text { Bristow Elementary School } & 86.2 \\ 61 & \text { Warren } & \text { Cumberland Trace Elementary School } & 102.6 \\ 62 & \text { Warren } & \text { Lost River Elementary School } & 90.1 \\ 63 & \text { Warren } & \text { North Warren Elementary School } & 97.5 \\ 64 & \text { Warren } & \text { Oakland Elementary School } & 101.8 \\ 65 & \text { Warren } & \text { Plano Elementary } & 97 \\ 66 & \text { Warren } & \text { Rich Pond Elementary School } & 104.2 \\ 67 & \text { Warren } & \text { Richardsville Elementary School } & 89.3 \\ 68 & \text { Warren } & \text { Rockfield Elementary School } & 97 \\ 69 & \text { Warren } & \text { Warren County Elementary School } & 74.7 \\ 70 & \text { Warren } & \text { William Natcher Elementary School } & 98.2\end{array}$




\section{APPENDIX I \\ IRB and HSRB Approval}

Subject: BRAAN2: IRB Protocol Marked as Exempt

Date: Wednesday, January 21, 2009 4:46 PM

From: InstitutionalReviewBoard@louisville.edu

To: jeanne.fiene@wku.edu, winifred.cohron@wku.edu

The following IRB Protocol has been marked as Exempt.

Tracking \#: 08.0638

PI: Fiene, Jeanne

Title: The Effect of Teacher Leader Interactions With

Teachers on Student Achievement: A Predictive Study

Link to BRAAN2 Login <https://braanprod.louisville.edu/> Help is available at the BRAAN2 Help Site <http://

louisville.edu/research/braan2>

For additional assistance please call the Human Subjects

Protection Program at 502-852-5188. 


\section{wo}

\section{WKU}

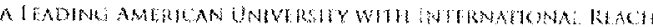

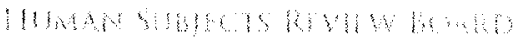

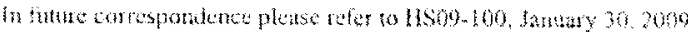

Dr. Starme Fuma:

Winitred Cohror

Educational Admmistranon, Leatenship, and Rowsarel

WKV

Dr. Jtanue Fienne

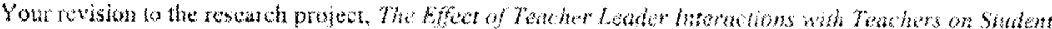

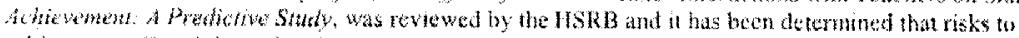

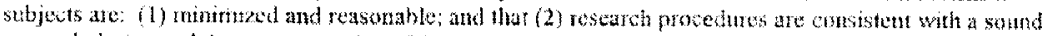

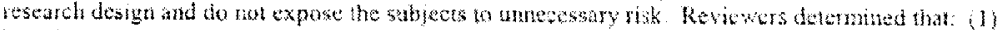

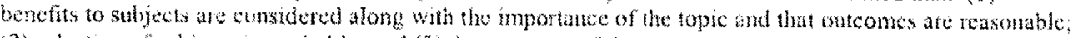

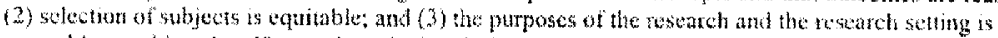

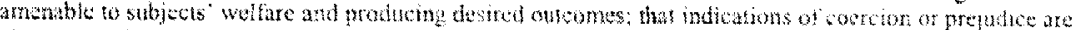
absent, and that particinaton is clearly whantary.

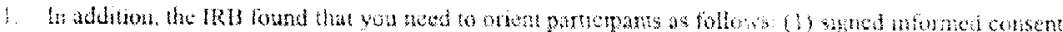

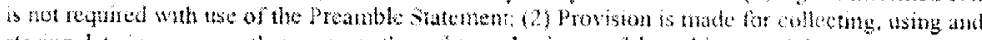
stonig data in a maner the protects the sately and privacy al the subects and the confindentiality of

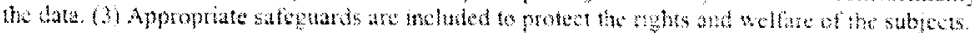

This pojet is therotore approved at the kipedited Review Level until dute $30,2009$.

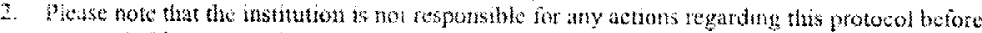
approval. If you expand the project at atce date to nse ofher instruments please re-apply. Copes of

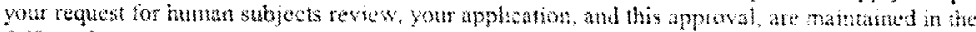
Offec of sponsored Progtans at he atowe address. Please repon any changes bo this appoved

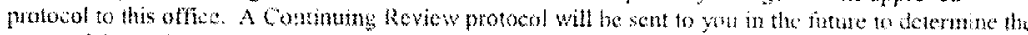

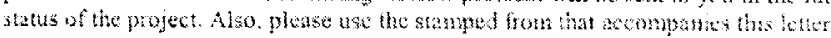

Sincerally,

Pul Mochay, MST.M

Complane Manager

Offec of Sponsored Proprams

Hestern Kurntacky Unversiy

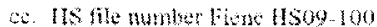

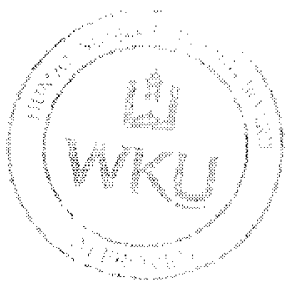

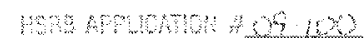

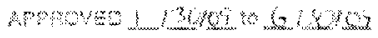

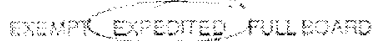

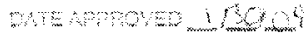

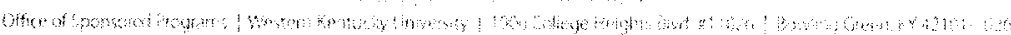

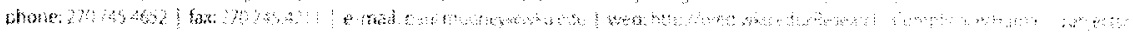
and 


\title{
APPENDIX J
}

\section{Permission to Use Survey}

\author{
Subj: $\quad$ Re: Survey Permission Request: W. Cohron, WKU, Bowling Green, KY \\ Date: $\quad$ 8/16/2008 12:13:29 PM Central Daylight Time \\ From: LinLambert \\ To: Rineman
}

Winnie,

Sorry for my delay in answering. I've been out of town with family, but am now in possession of both your e-mail and your letter. I celebrate your efforts on this dissertation and am very excited about it.

Formally, you should get permission to use the survey from:

Chris Richards, Permissions depl.

Crichard@ascd.org

Just tell him that it's fine with me.

I'm surprised that you can't find the literature that you need on teacher leadership. Have you checked out the work of Ann Lieberman and Gayle Moller? Also, see my recent writings on Lasting Leadership and my leadership capacity study. Check out publications on my vita on www.Lambertleadership.org.

Unfortunately, I did not do statistical tests on the surveys, although I piloted them and refined them many times. There have been many dissertations done with this work. Hopefully you can find them on-line. I retired a few years ago (am now writing novels) and no longer keep track of these studies.

I'm afraid that I haven't been very helpful, but good luck to you, Linda 
In a message dated 8/26/2008 12:58:42 PM Central Dayllght Time, permissions@ascd.org writes:

Dear Ms. Cohron,

In response to your request dated August 17,2008, ASCD grants you the one-time non-exclusive right to utilize and adapt the following ASCD materials ("Material") as a survey tool for your dissertation.

Lambert. Linda (1998) Leadership Capacity School Survey. Building Leadership Capacity in Schools (pp. 100-105). Alexandria, VA: ASCD.

This permission covers the fext portion of the Material only and does not extend to content that is separately copyrighted. Please note that it is your responsibility to secure permisston for any text, photographs, illustrations, cartoons, advertisements, etc. that are referenced to another source. The reproduction of covers, mastheads, and logos of ASCD publications is strictly prohiblted.

Permission is limited to your use as described above, and does not include the right (a) to grant others permission to photocopy or othenwise reproduce the Material, except for versions made by non-protit organizations for use by blind or physically handicapped persons, provided that no fees are charged, nor (b) to reproduce the Material in digital, electronic, or any other media.

No fee is required for this use, however, permission is granted upon the condition that every copy of the Material distributed contains a full acknowledgment including: title, author(s) and/or editor(s), journal or book title, including volume/issue/date (if applicable), the identical copyright notice as it appears in our publication, the legend "Reprinted by Permission. "Learn more about ASCD at www, ascd.org."

We would appreciate your acknowledging the above by return email. Otherwise, thank you for your interest in ASCD publications.

Sincerely,

Kat Rodenhizer

Coordinator for Rights \& Permissions

ASCD

703-575-5443

http://www.ascd.oro 


\section{CURRICULUM VITAE}

Winifred Riney Cohron

940 Kensington Way

Bowling Green, Kentucky 42103

270-781-8394

E-mail: Winifred.cohron@ awarren.kyschools.us

\section{Education}

2009 University of Louisville and

Western Kentucky University

2005 Western Kentucky University

1988 Western Kentucky University

1983 Loyola University of Chicago

1974 Brescia College
Ph.D, Educational Leadershp

Rank 1, Educational Administration

Rank I, Middle Grades Social Studies

Master of Religious Education

Bachelor of Arts, Magna cum laude

\section{Certifications}

Kentucky Certified Supervisor of Instruction

Kentucky Certified Principal for All Grades

Kentucky High School History for Grades $7-12$

Kentucky Social Studies Program Consultant

\section{Professional Employment}

\section{7 - Present}

Supervisor of Instruction, Middle and High Schools

Warren County Board of Education

Bowling Green, KY

- District Curriculum Mapping/Student Learning Target Coordinator (6 - 12)

- District Carl D. Perkins Grant Coordinator

- Kentucky Tech Prep State Advisory Committee

- District Individual Learning Plan (ILP) Coordinator

- District Governor's Scholar Coordinator

- District Commonwealth Diploma Coordinator

- Comprehensive District Improvement Plan (CDIP) Committee Member

- SACS Evaluator

- Scholastic Audit Team Member

- Instructor - WKU Instructional Strategies Workshop for WKU graduate credit

- WKU Masters in Education Re-Design Committee Member

2004-2007

Assistant Principal 
Drakes Creek Middle School

Bowling Green, Kentucky 42104

- Teacher Observation and Evaluation

- Assessment Coordinator

- Test Data Analysis

- Comprehensive School Improvement Committee Chair

- Curriculum Coordinator

- ESS Coordinator

- Textbook Adoption Coordinator

- SBDM Secretary

- Curriculum Committee Chair

- Leadership Team Facilitator

- Professional Development Coordinator

- SACS Evaluator Team Member

- District Scholastic Audit Team Member

- 2006 Participant in WKU Hayes-Fulbright Study Group to China

2001-2004

Curriculum Coordinator

Drakes Creek Middle School

Bowling Green, Kentucky 42104

- Assessment Coordinator

- Test Data Analysis

- Textbook Adoption Coordinator

- Curriculum Committee Chair

- Professional Development Coordinator

- Comprehensive School Improvement Chair

- Parent Volunteer Liason

- District Scholastic Audit Team Member

- District 6-8 Vertical Alignment Facilitator

- WKU Writing Project XVIII Participant

1996-2001

Drakes Creek Middle School

Bowling Green, Kentucky 42104

- Eighth Grade Social Studies Teacher

- Part-time Curriculum Coordinator

- Curriculum Committee Chair

- Comprehensive School Improvement Committee Chair

- SACS Preparation Committee Chair

- Kentucky Council for Social Studies Steering Committee Member 
- District Social Studies Alignment Committee Co-Chair

- Region 2 Social Studies Academy Participant

- Kentucky Department of Education Middle Grades Social Studies Core Benchmarking Contributor and Standards Setting Process Contributor

- Social Studies Textbook Adoption Chair

- Kentucky Department of Education Content Advisory Committee Member

1988-1995

Eighth Grade Social Studies Teacher

Drakes Creek Middle School

- Quick Recall and Academic Team Coach

- Team Leader

- Student Teaching Resource Teacher

- Principal Selection Committee

$1984-1988$

Seventh and Eighth Grade Social Studies Teacher Cumberland Trace Elementary School

- Quick Recall and Academic Team Coach

- Kentucky Junior Historical Society Sponsor

1974-1984

Parochial school teacher in Kentucky and Tennessee

\section{Presentations}

November 2008 - Writing Effective Open Response Questions

Warren East High School English Department, Bowling Green, KY

October 2008 - Research Supporting Teacher Leader Masters in Education Re-Design Western Kentucky University College of Education Faculty, WKU, Bowling Green, KY

August 2007, 2008 - Best Practice in Assessment

Warren County Schools New Teacher Orientation, Bowling Green, KY

June 2007 - Using the Reading for Meaning Strategy in the Classroom

The Art and Science of Teaching Institute (Thoughtful Education Associates)

Lexington, $\mathrm{KY}$

May 2007 - Transitioning Experience into Professional Practice: Meanings of a China StudyTrip. 2007 Kentucky Conference on the Scholarship of Teaching and Learning: Engaging Campus and Community, Lexington, $\mathrm{KY}$

September 2006 - Successful Practices for Student Achievement Kentucky Association of School Councils Annual Conference, Louisville, KY 
August 2006 - Thoughtful Education Strategies as Implemented in Warren County Schools WKU College of Education Faculty, WKU, Bowling Green, KY

July 2003 - Writing Open Response Questions

Lost River Elementary School, Bowling Green, KY

February 2003 -- Preparing for Proficiency

Kentucky Council of Teachers of English/Language Arts Conference, Lexington, KY

September 2002 - Successful Kentucky Middle Schools Showcase

Kentucky Middle School Association Annual State Conference, Lexington, KY

September 2002 - Preparing for Proficiency

Kentucky Council for the Social Studies Annual Conference, Louisville, KY

July 2002 - Curriculum Mapping

Breckenridge County Schools, Hardinsburg, KY

May 2000 - Writing Open Response Questions, Warren County Schools Professional Development, Bowling Green, KY

January 2000 - Writing Open Response Questions, Caverna Schools, Caverna, KY

\section{Publications}

Cohron, W. (1996, Fall). Enlivening History in Warren County Schools. The Link, 39(3), 6-7

Create an Andrew Jackson Mural. (1998) In Appleby, J., Brinkley, A., and McPherson, J. (1998). The American Journey: Teachers Wraparound Edition. (p. 332b).

Glencoe/McGraw-Hill: New York.

\section{Awards}

Campbellsville University Excellence in Teaching Award 1999

Kentucky Council for Social Studies Outstanding Teacher Award 1998

Warren County Middle School Teacher of the Year 1991

Drakes Creek Middle School Teacher of the Year 1990, 1992

\section{Professional Organizations}

Kentucky Association of School Administrators

Association of Supervision and Curriculum Development

National Staff Development Council

International Reading Association

Kentucky Association of Educational Supervisors 